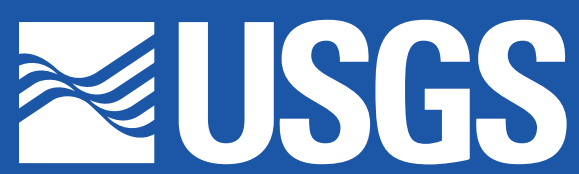

science for a changing world

\title{
Composition, Age, and Petrogenesis of Late Cretaceous Intrusive Rocks in the Central Big Belt Mountains, Broadwater and Meagher Counties, Montana
}

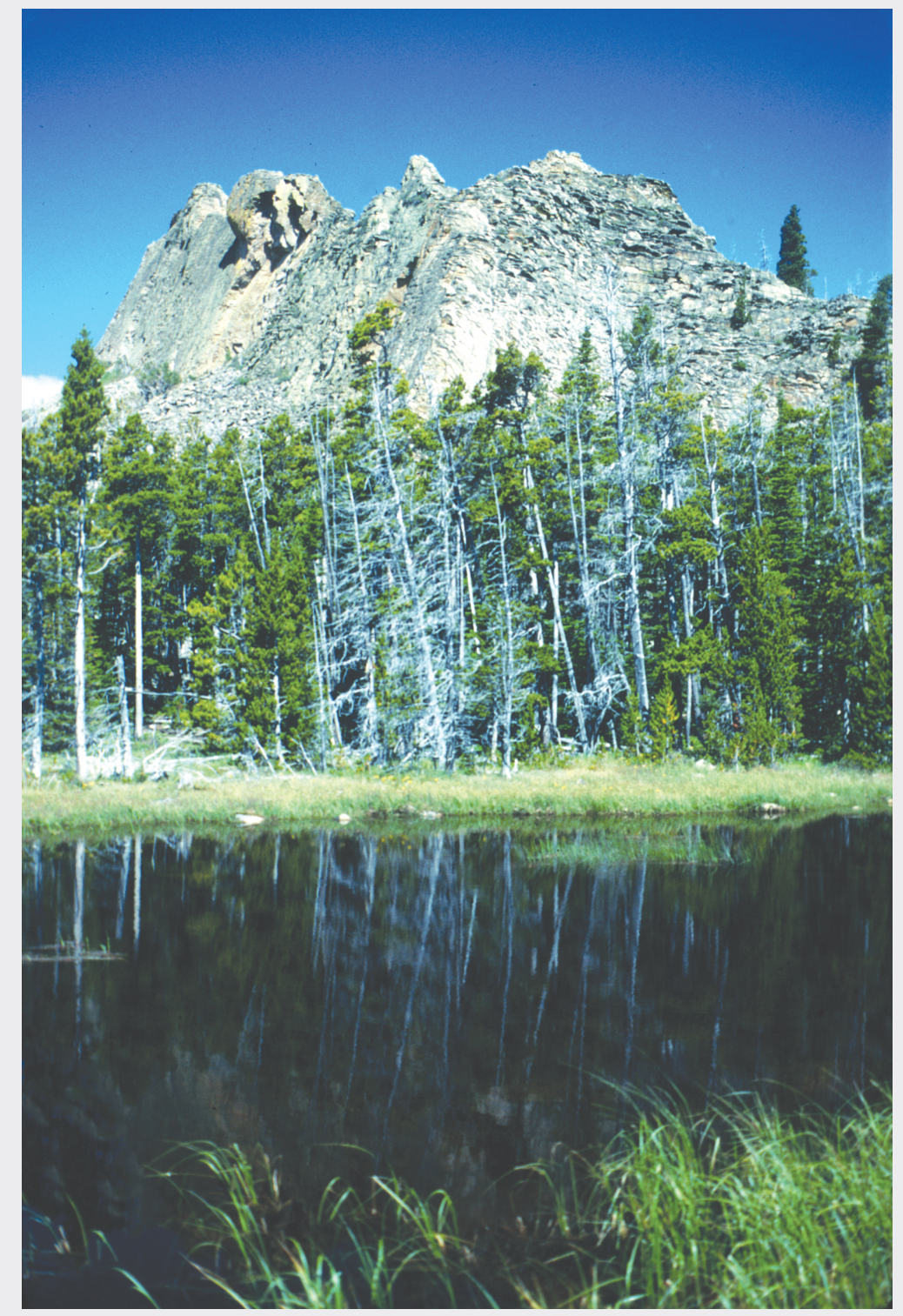

U.S. Geological Survey Professional Paper 1657 
Cover. The Needles, about 6 kilometers south-southeast of Gipsy Lake, form spectacular outcrops of the quartz monzodiorite of Mount Edith. 


\section{Composition, Age, and Petrogenesis of Late Cretaceous Intrusive Rocks in the Central Big Belt Mountains, Broadwater and Meagher Counties, Montana}

ByE.A. du Bray and L.W. Snee

U.S. Geological Survey Professional Paper 1657

U.S. Department of the Interior

U.S. Geological Survey 


\section{U.S. Department of the Interior Gale A. Norton, Secretary}

\section{U.S. Geological Survey \\ Charles G. Groat, Director}

This publication is also available online at:

http://geology.cr.usgs.gov/pub/ppapers/p1657/

Any use of trade, product, or firm names in this publicationÄ

is for descriptive purposes only and $\ddot{A}$

does not imply endorsement by the U.S. Government

Version 1.02002

Library of Congress Cataloging-in-Publication Data

\section{Du Bray, E.A.}

Composition, age, and petrogenesis of late Cretaceous intrusive rocks in the central Big Belt Mountains, Broadwater and Meagher counties, Montana / by E.A. du Bray and L.W. Snee.

p. cm. - (U.S. Geological Survey professional paper ; 1657) Includes bibliographical references.

1. Geology, Stratigraphic-Cretaceous. 2. Rocks-MontanaBroadwater County-Analysis. 3. Rocks-Montana-Meagher County-Analysis. I. Snee, Lawrence W. II. Title. III. Series. QE688.D8 2002

$557.86^{\prime} 612-\mathrm{dc} 21$

2002023527 


\section{Contents}

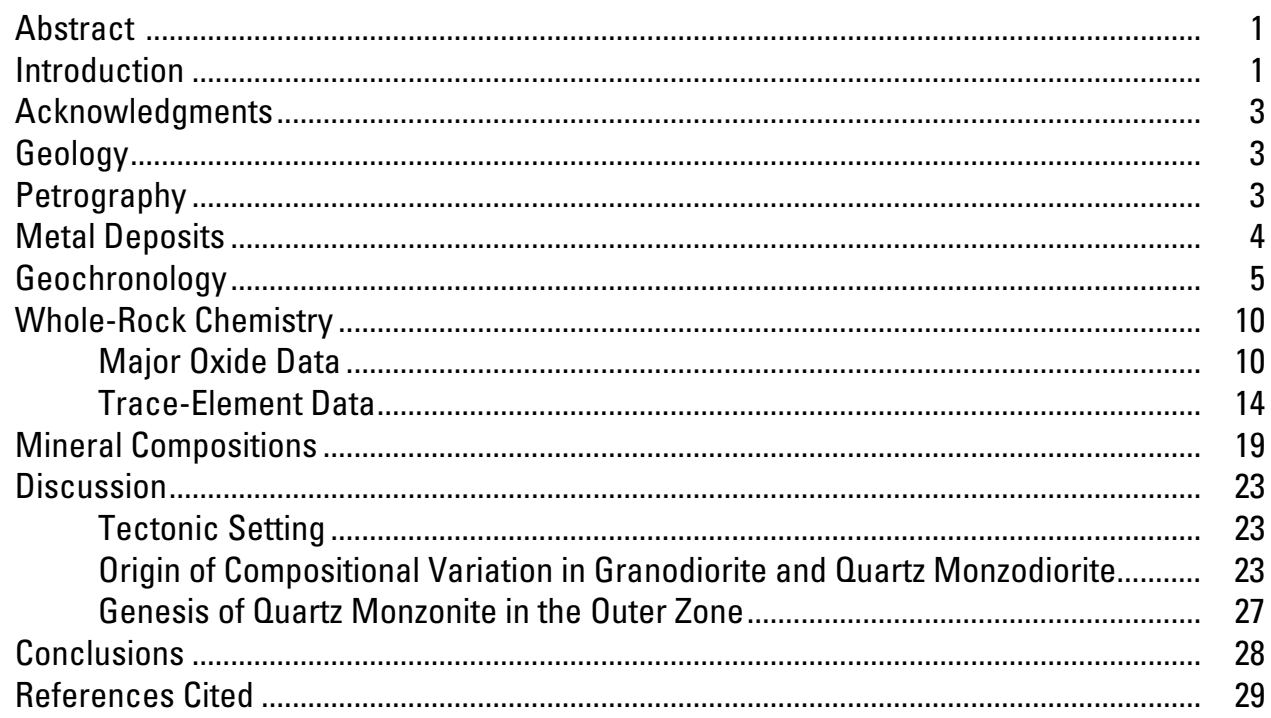

\section{Figures}

1. Geologic map showing distribution of Late Cretaceous intrusive rocks in central Big Belt Mountains, Montana.

2. Quartz-potassium feldspar-plagioclase ternary diagram showing modal compositions of central Big Belt Mountains intrusions.

3. Diagrams showing ${ }^{40} \mathrm{Ar} /{ }^{39} \mathrm{Ar}$ age spectra for separates from intrusions in central Big Belt Mountains

4. ${ }^{40} \mathrm{Ar} /{ }^{36} \mathrm{Ar}$ versus ${ }^{39} \mathrm{Ar} /{ }^{36} \mathrm{Ar}$ isotope correlation diagrams for biotite and hornblende samples 1 3 10 10 14 19 23 23 23 27 28 29

5. Variation diagrams showing abundances of major oxides and zirconium in intrusions in central Big Belt Mountains...

6. Total alkali-silica variation diagram for intrusions of central Big Belt Mountains.

7. Variation diagram showing relative proportions of $\mathrm{K}_{2} \mathrm{O}, \mathrm{Na}_{2} \mathrm{O}$, and $\mathrm{CaO}$ in intrusions of central Big Belt Mountains.

8. Chondrite-normalized extended trace-element diagrams showing compositions of central Big Belt Mountains intrusions compositions of central Big Belt Mountains intrusions

10. Ternary diagram showing molecular end-member proportions of feldspars in samples of central Big Belt Mountains intrusions 
11. Ternary $M g, \mathrm{R}^{+3}\left(\mathrm{Al}^{\mathrm{IV}}+\mathrm{Fe}^{3+}+\mathrm{Ti}\right), \mathrm{R}^{+2}\left(\mathrm{Fe}^{2+}+\mathrm{Mn}\right)$ diagram showing relative cation abundances for biotite in samples of central Big Belt Mountains intrusions

12. Ternary diagram showing compositions of amphibole and clinopyroxene in quartz monzodiorite of central Big Belt Mountains intrusions

13. Trace-element tectonic-setting-discrimination variation diagram for central Big Belt Mountains intrusions

14. Ternary AFM $\left(\mathrm{Na}_{2} \mathrm{O}+\mathrm{K}_{2} \mathrm{O}-\mathrm{FeO} \mathrm{O}^{*}-\mathrm{MgO}\right)$ diagram showing compositions of central Big Belt Mountains intrusions

15. Th/Sm vs. Th variation diagram showing compositions of central Big Belt Mountains intrusions

16. Extended trace-element abundance diagram showing compositions of central Big Belt Mountains intrusions

17. Ternary variation diagram showing relative proportions of $\mathrm{Rb}, \mathrm{K}$, and $\mathrm{Sr}$ in granodiorite and quartz monzodiorite in central Big Belt Mountains

18. Rare-earth element diagram showing compositions of central

Big Belt Mountains intrusions

\section{Tables}

1. Summary of ${ }^{40} \mathrm{Ar} /{ }^{39} \mathrm{Ar}$ age-spectrum results for intrusive rocks from central Big Belt Mountains, Mont.

2. ${ }^{40} \mathrm{Ar} /{ }^{39} \mathrm{Ar}$ incremental heating data for samples from central

Big Belt Mountains

3. Composition of Cretaceous intrusive rocks from central Big Belt Mountains

4. Instrumental neutron activation analysis of selected samples of Cretaceous intrusive rocks from central Big Belt Mountains

5. Major oxide compositions of plagioclase in intrusive rocks from central Big Belt Mountains

6. Compositions of potassium feldspar in intrusive rocks from central Big Belt Mountains

7. Compositions of biotite in intrusive rocks from central Big Belt Mountains...

8. Compositions of hornblende in intrusive rocks from central Big Belt Mountains ..... 21

9. Compositions of clinopyroxene in intrusive rocks from central Big Belt Mountains

10. Representative compositions of accessory phases in intrusive rocks from central Big Belt Mountains.

11. Results of major oxide least-squares crystal fractionation-mixing modeling for intrusive rocks from central Big Belt Mountains. 


\title{
Composition, Age, and Petrogenesis of Late Cretaceous Intrusive Rocks in the Central Big Belt Mountains, Broadwater and Meagher Counties, Montana
}

\author{
By E.A. du Bray and L.W. Snee
}

\begin{abstract}
The central Big Belt Mountains in western Montana contain two Late Cretaceous intrusions that are satellitic to the Boulder batholith. New ${ }^{40} \mathrm{Ar} /{ }^{39} \mathrm{Ar}$ data demonstrate that these intrusions, their hydrothermally altered margins, and small peripheral metal deposits are temporally related, which indicates a genetic link among their causative magmato-hydrothermal processes. These deposits and associated altered rocks are similar to other recently described, globally dispersed intrusionrelated gold systems. As such, rocks in the central Big Belt Mountains help better define the physical and genetic characteristics of these deposits and establish the petrogenesis of their host granitoid intrusions.
\end{abstract}

\section{Introduction}

The Big Belt Mountains are in western Montana about 50 $\mathrm{km}$ southeast of Helena (fig. 1). In contrast to igneous rocks in nearby areas, especially the Elkhorn Mountains Volcanics (Rutland and others, 1989) and the Boulder batholith (Tilling, 1973) to the west, very little is known about intrusions exposed in the central Big Belt Mountains. A geologic map of the Confederate Gulch area and accompanying tabulation of regional geochemical data (Gualtieri, 1975a, b) are the sole published geologic information for the northern of the two principal intrusions in this area. The geology of the Duck Creek Pass quadrangle, immediately south of the area mapped by Gualtieri (1975a, b), was described by Nelson (1963), whose map provides geologic data for the extreme west edge of the central Big Belt Mountains.

Geologic mapping of the central Big Belt Mountains (du Bray, 1995a) documents the distribution of intrusions exposed in this area. These intrusions and their associated altered and mineralized rocks are an important research target because they are similar to recently described intrusions and associated gold deposits located elsewhere in the world (Lang and others, 2000, and references contained therein). Characterization of these intrusions, including their geochemistry and geochronology, and the deposits they host presents an opportunity to put these rocks in a global context and to establish the tectonic and petrogenetic processes that controlled their evolution.

The intrusive suite of Boulder Baldy, composed of quartz monzodiorite and granodiorite, constitutes the northern intrusion; the southern intrusion, the quartz monzodiorite of Mount Edith, was previously unknown. New ${ }^{40} \mathrm{Ar} /{ }^{39} \mathrm{Ar}$ geochronologic data indicate that magmas represented by these rocks solidified sometime before $68.5 \mathrm{Ma}$, that all are part of a single magmatic event, and that regional cooling to below $150^{\circ} \mathrm{C}$ subsequent to magmatism was complete by about $60 \mathrm{Ma}$. Geochemical and geologic relations suggest that these calcalkaline intrusions are related to subduction and back-arc processes that prevailed along the late Mesozoic margin of western North America. Mixing calculations indicate that compositional variation, from mafic to felsic quartz monzodiorite and granodiorite, developed by crystallization and removal of varying amounts of plagioclase, clinopyroxene, hornblende, titanite, apatite, and magnetite, the principal constituents of these rocks. Solidification of the respective magma reservoirs involved crystal nucleation along bounding surfaces; residual silicate liquid at crystallization fronts separated from early-formed solids and remixed with undepleted magma away from crystallization fronts to produce progressively more evolved magma.

Unusual textures, mineralogy, and erratic compositional variation in the outer part of the intrusive suite of Boulder Baldy reflect extensive hydrothermal, metasomatic alteration and recrystallization of quartz monzodiorite to quartz monzonite by metal-bearing fluids. Hornblende was replaced by aegirine-augite, which in turn, in the most strongly altered rocks, was replaced by andradite. The abundance of fluorite in these rocks and the fact that these rocks are hosted by very fine grained Newland Formation that lacks significant permeability suggest that fluids responsible for the alteration were primarily magmatic. Textural features indicate that magma represented by rock in the outer part of the intrusive suite was pressure quenched, which may have caused rapid exsolution of fluids responsible for the observed alteration. The intensity of alteration is correlated with the extent of pressure quenching; quartz monzodiorite of Mount Edith, in which pressure quenching was limited and restricted to rock near bounding contacts, is unaltered. 


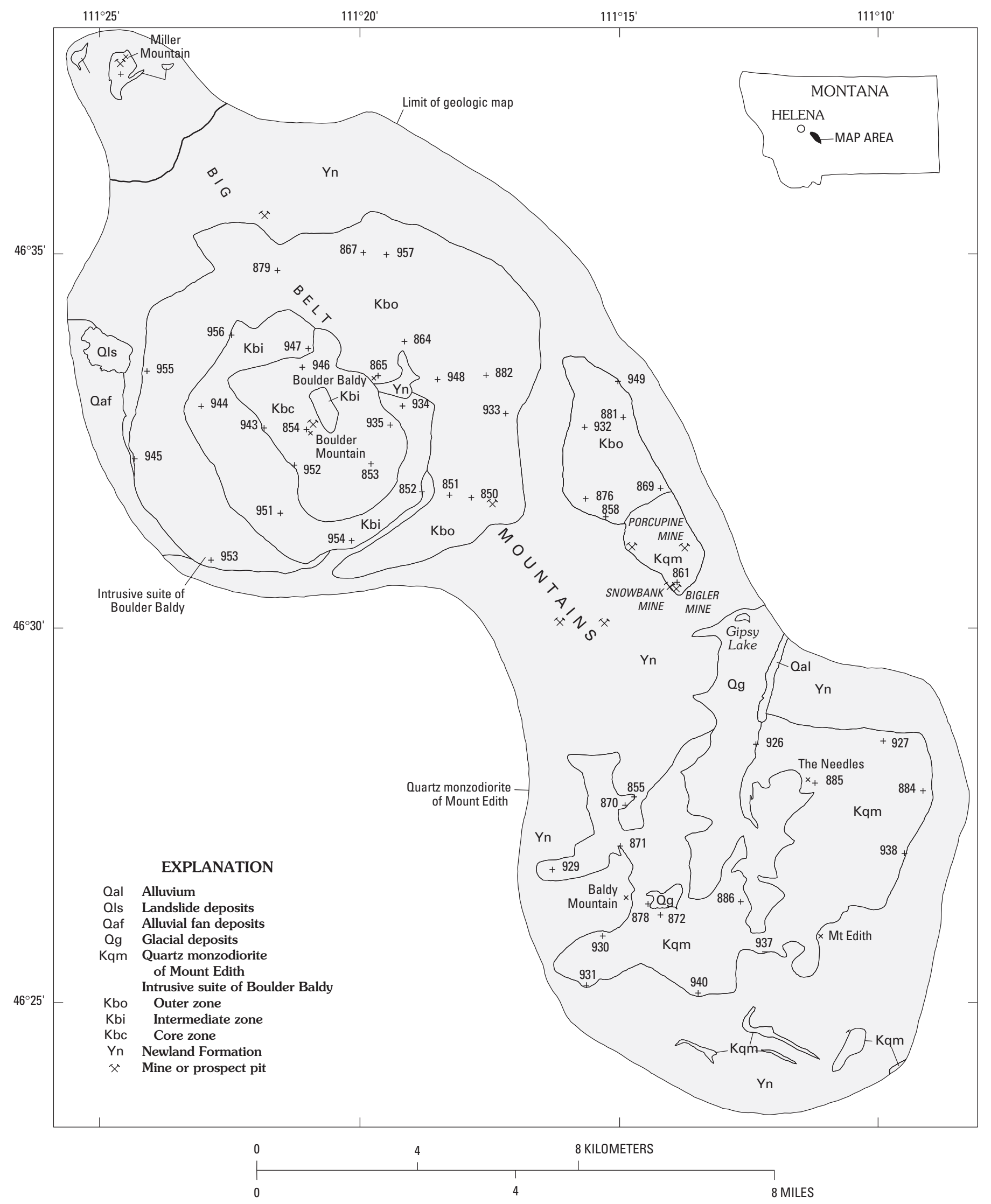

Figure 1. Distribution of Late Cretaceous intrusive rocks in central Big Belt Mountains, Mont. (du Bray, 1995a). Sample numbers (at localities indicated by plus signs) must be prefaced by 202 to obtain complete designations. 


\section{Acknowledgments}

Field work was facilitated by Beth Ihle of the U.S. Forest Service, who helped obtain access to Forest and adjacent lands. Local landowners are thanked for providing access across their lands. Eugene E. Foord helped identify andradite garnet in some of the analyzed samples. Ross Yeoman conducted ${ }^{40} \mathrm{Ar} /{ }^{39} \mathrm{Ar}$ analyses. Technical reviews by Margaret Hiza and David John improved this report.

\section{Geology}

The Big Belt Mountains are underlain principally by Middle Proterozoic sedimentary rocks of the Belt Supergroup, mostly the Middle Proterozoic Newland Formation; other Belt Supergroup formations are also present. Between Confederate Gulch and the southern slopes of Mount Edith (fig. 1), the crest of the central Big Belt Mountains is composed largely of granitoid intrusions that are satellitic to the nearby Boulder batholith (Tilling, 1973). Gualtieri's (1975a) map shows an approximately circular, 10-km diameter intrusion centered between Boulder Baldy and Boulder Mountain and a smaller satellitic stock $1.5 \mathrm{~km}$ to the east. A large pluton, the quartz monzodiorite of Mount Edith, crops out north of Mount Edith and east of Baldy Mountain (du Bray, 1995a). Nelson (1963) mapped three small stocks arrayed along the west flank of the range in the north-central part of the Duck Creek Pass quadrangle. Mapping by du Bray (1995a) shows that these three stocks are actually contiguous apophyses of the quartz monzodiorite of Mount Edith. Geochronologic data compiled by Daniel and Berg (1981) indicate potassium-argon dates (recalculated using new constants of Steiger and Jäger, 1977, and tables of Dalrymple, 1979 ) of $64.4 \pm 2.4 \mathrm{Ma}$ (biotite) and $71.5 \pm 3.2 \mathrm{Ma}$ (hornblende) for a pluton, several kilometers to the west, that is petrographically similar to quartz monzodiorite intrusions of the central Big Belt Mountains. Several factors, including close spatial association and petrographic similarities, suggest that all the plutonic rocks exposed along the crest of the central Big Belt Mountains are genetically related and part of a single magmatic episode. Gravity and aeromagnetic data (A.E. McCafferty, written commun., 1994) further indicate the subsurface continuity of central Big Belt Mountains intrusions.

Mapping by Gualtieri (1975a) accurately shows the geologic relations of the somewhat unusual, concentrically zoned, and generally poorly exposed intrusive suite centered between Boulder Baldy and Boulder Mountain. His map indicates three zones: (1) an outer zone composed of a syenite complex, including several textural variants, (2) an intermediate zone composed of monzonite porphyry, and (3) a core zone composed of monzonite. Mapping by du Bray (1995a) confirms that the Late Cretaceous intrusive suite of Boulder Baldy is a $10-\mathrm{km}$ diameter intrusion composed of three concentrically arranged, compositionally and texturally distinct zones. Relative age relations among the three zones of this intrusion are indeterminate; similarly zoned plutons (Bateman, 1992), are youngest in their central parts, however. The textural and compositional diversity of the outer 1.5- to 5-km wide zone of the intrusive suite is unusual, as is its spatial and apparent temporal and genetic associations with undistinctive calc-alkaline rock that forms the inner two zones. The textures and compositions of the outer zone rocks are not those of normally solidified granitoid rock; rather, they are like those of solidified and subsequently hydrothermally altered granitoid rock. The intermediate and core zones are composed of granitoid rock petrographically and compositionally similar to other calc-alkaline Cretaceous intrusions (the Boulder batholith, for instance) elsewhere in western Montana, and to calc-alkaline plutons that coalesce to form Mesozoic batholiths throughout the North American Cordillera. The annular intermediate zone is 0 to $2.5 \mathrm{~km}$ wide, and the elliptical core zone is $2.5 \mathrm{~km}$ wide and has a northwest-trending axis about $5 \mathrm{~km}$ long.

A small stock at the southeast edge of Gualtieri's (1975a) map is part of a generally elliptical, $5-\mathrm{km}$ long by $2.5-\mathrm{km}$ wide north-northwest-trending intrusion whose south end is about 1 $\mathrm{km}$ northwest of Gipsy Lake (fig. 1). The northern two-thirds of this stock is composed of rock similar to that which forms the outer zone of the intrusive suite of Boulder Baldy. The southern third is composed of rock similar to that which forms the quartz monzodiorite of Mount Edith and the intermediate zone of the intrusive suite.

The area about $1 \mathrm{~km}$ northwest of Confederate Gulch contains three small stocks (Kbc, fig. 1) that are mineralogically and compositionally similar to rock forming the core zone of the intrusive suite of Boulder Baldy (Kbc at Boulder Baldy, fig. 1). The largest of these stocks, centered on Miller Mountain (coincident with sample 883 , fig. 1), has been extensively prospected as a lode-gold source. The stock is cut by a stockwork consisting of numerous high-angle and crosscutting horizontal quartz veins. All the stocks exposed in this area are considered to be apophyses of the much larger intrusive suite of Boulder Baldy.

The quartz monzodiorite of Mount Edith forms an essentially undocumented pluton that is generally circular and $10 \mathrm{~km}$ in diameter. It is composed of quartz monzodiorite that is petrographically and compositionally similar to rock that forms the intermediate zone of the intrusive suite of Boulder Baldy. The close compositional and spatial association between quartz monzodiorite in the two plutons of the central Big Belt Mountains implies a possible common genesis. Most of the quartz monzodiorite of Mount Edith is covered by a mantle of glacial deposits. Only at The Needles (fig. 1), and in cirque walls north of Mount Edith and north-northeast of Baldy Mountain, is this pluton well exposed.

\section{Petrography}

Rock descriptions and classification in this study are a synthesis of petrographic examinations and stained slab modal analyses (du Bray, 1995a). Plutonic rock names are in accord with the IUGS system (Streckeisen, 1973). Indicated crystal sizes are approximate averages for their maximum dimensions. All mineral colors refer to the primary color noted in thin sections viewed in plane polarized light. Specific mineral names are based on electron microprobe composition data. 
Granodiorite (inner zone) of the intrusive suite of Boulder Baldy (fig. 2) is fine to medium grained and hypidiomorphic equigranular. Its color index is about 6; yellowish-brown to dark-brown anhedral to subhedral biotite $(0.6 \mathrm{~mm})$ is the sole mafic silicate mineral. Quartz $(1 \mathrm{~mm})$ is anhedral and weakly strained. Potassium feldspar $(1.2 \mathrm{~mm})$ and plagioclase $(1.2 \mathrm{~mm})$ are zoned, twinned, and subhedral. Trace constituents are magnetite, titanite, and apatite.

Hornblende quartz monzodiorite (intermediate zone) of the intrusive suite of Boulder Baldy is medium grained, hypidiomorphic inequigranular to seriate textured, and it contains distinctive, euhedral, perthitic phenocrysts of potassium feldspar as much as $3 \mathrm{~cm}$ long. Its color index is about 8 , and subhedral yellow-green to dark-green hornblende $(1.2 \mathrm{~mm})$ is the sole mafic silicate mineral. Quartz $(0.6 \mathrm{~mm})$ is anhedral and interstitial. Subhedral plagioclase $(1.7 \mathrm{~mm})$ is twinned and zoned. Trace constituents are titanite, magnetite, apatite, and zircon. In some places, the quartz monzodiorite is characterized by a distinctly fine grained, anhedral groundmass that is interstitial to otherwise coarser grained and better formed crystals. The amount of interstitial groundmass is highly variable between samples.

Aegirine-augite quartz monzonite (outer zone) of the intrusive suite of Boulder Baldy is compositionally and texturally diverse alkali-feldspar-rich, syenitoid rock that ranges to monzodiorite, monzonite, syenite, quartz syenite, and alkali-feldspar syenite (fig. 2). Anhedral quartz $(0.5 \mathrm{~mm})$ is scarce to absent in much of this rock. A fine-grained, quenched or recrystallized groundmass is a nearly ubiquitous feature of this nearly equigranular to coarsely porphyritic rock. In contrast, the outer zone in some places is composed almost exclusively of aligned, densely packed, euhedral perthite phenocrysts as much as $6 \mathrm{~cm}$ long. The distribution of this variant is highly irregular; delineation of its extent is not possible because of notably sparse outcrop. In yet other places, the outer zone consists of intermingled pegmatitic and aplitic rock. The color index of the quartz monzonite is about 3; anhedral, emerald-green aegirine-augite to aegirine $(0.5 \mathrm{~mm})$ is the sole mafic silicate mineral. This rock type contains trace amounts to several modal percent anhedral andradite and abundant subhedral titanite. Some andradite is associated spatially with small altered grains of clinopyroxene that it appears to have replaced. Some samples contain trace amounts to several modal percent of widely disseminated purple fluorite. Trace constituents are magnetite, apatite, and zircon.

The quartz monzodiorite of Mount Edith is composed of perthite phenocryst-bearing hornblende quartz monzodiorite that is petrographically and compositionally similar to the intermediate zone of the intrusive suite of Boulder Baldy. Its color index is about 6; subhedral yellow-green to dark-green hornblende (1 $\mathrm{mm})$ is the principal mafic silicate mineral. Quartz $(0.6 \mathrm{~mm})$ is anhedral and weakly strained. Plagioclase $(2 \mathrm{~mm})$ is subhedral, twinned, and zoned. Trace constituents are titanite, magnetite, apatite, and zircon. Like the quartz monzodiorite of the intrusive suite of Boulder Baldy, the quartz monzodiorite of Mount Edith locally includes a groundmass that is fine grained and anhedral; this type of groundmass is restricted to rock near the contact between the intrusion and enclosing Newland Formation rocks, however. A mafic border phase, represented by sample No. 202940 (table 3), is present at the outer edge of this intrusion in

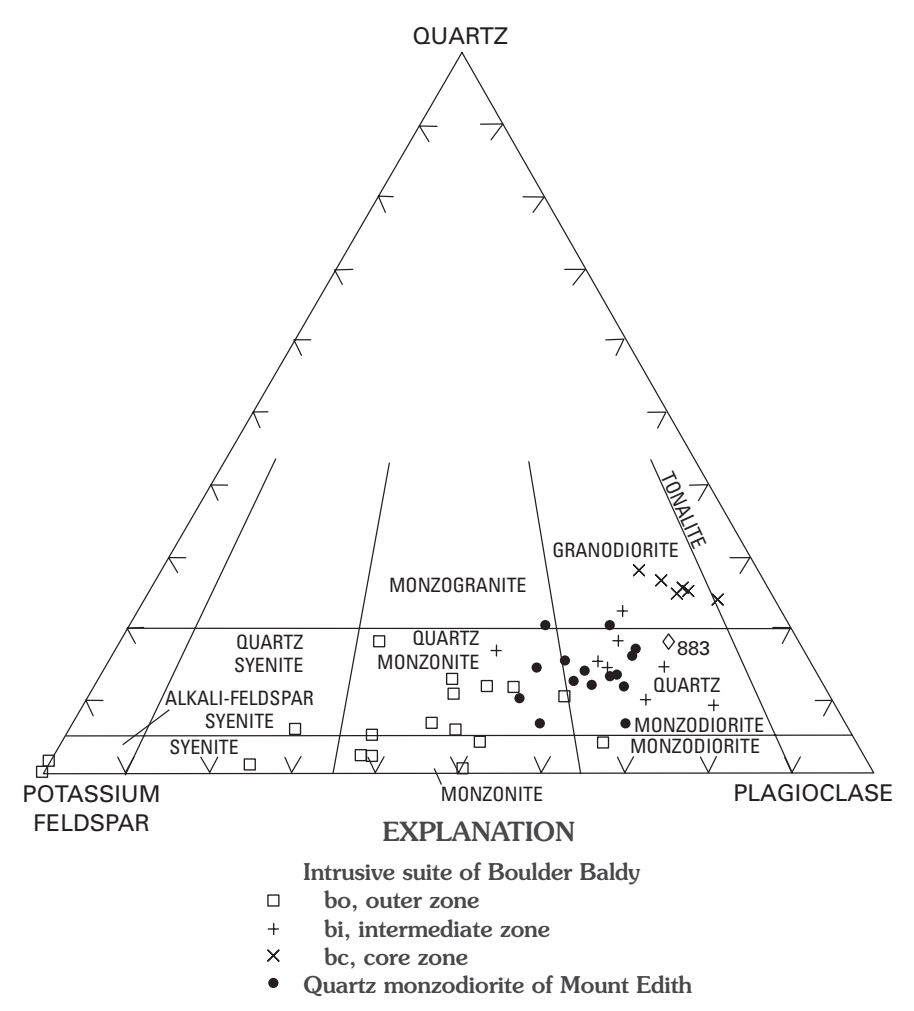

Figure 2. Modal compositions of central Big Belt Mountains intru sions. Sample 883 (202883) is from the small stock exposed at Miller Mountain (fig. 1). Classification grid and rock names are those of Streckeisen (1973).

some places. This phase is characterized by dense accumulations of aligned plagioclase, by elevated hornblende content, and by trace amounts of clinopyroxene; these rocks are probably cumulates.

\section{Metal Deposits}

During the latter half of the 1800's, and sporadically thereafter, the Confederate Gulch area (fig. 1) was a major producer of placer gold (Lyden, 1948). Lyden (1948) estimated that 6-18 million dollars worth of gold was produced from placer deposits in Confederate Gulch before 1933. McClernan (1980) showed numerous sets of placer workings along drainages whose headwaters are coincident with the intrusive suite of Boulder Baldy. The gold in these deposits is attributed commonly to lode sources at Miller Mountain (fig. 1) on the north side of Confederate Gulch (Lyden, 1948). Miller Mountain has been the site of recent lode-gold exploration activities and was extensively prospected in previous times. A resource of at least 11 million tons of ore containing $310,000 \mathrm{oz} \mathrm{Au}$ (grade, $0.028 \mathrm{oz} /$ ton) was delineated at Miller Mountain by a Pegasus Gold Corporation/ FMC Gold Company joint venture drilling program (Pegasus Gold Inc., 1992). Widely disseminated gold-mineralized rock is hosted by weakly altered, sulfide-poor (pyrite), granitic rock at Miller Mountain. Gold grades are highest near major structural 
intersections, particularly along low-angle quartz vein shear zones, in granodiorite exposed at Miller Mountain. A close spatial association between mineralized and intrusive rocks in the central Big Belt Mountains implies a genetic relationship.

Altered Newland Formation from a prospect pit $5 \mathrm{~km}$ north northeast of Boulder Baldy (fig. 1) contains anomalous but low abundances of silver, arsenic, bismuth, cadmium, and lead. Igneous rock exposed 3-4 km west of Gipsy Lake contains several shallow prospect pits and trenches, is bleached and locally contains fluorite in veins and disseminations, and contains anomalous abundances of silver, bismuth, and cadmium.

The Snowbank and Bigler mines lie along the contact between the south end of the satellitic stock in the Gipsy Lake area and enclosing Newland Formation, whereas the nearby Porcupine mine is in potassium feldspar porphyritic quartz monzodiorite (fig. 1). In all three settings the prospected material consists of iron-oxide coatings along fractures in the granitoid rock and in the Newland Formation near its contact with the granitoid rocks. Rock from these areas contains anomalous abundances of arsenic, silver, gold, bismuth, cadmium, copper, molybdenum, and lead in varying combinations. Rock from a small unnamed mine about $1.5 \mathrm{~km}$ west of the Porcupine mine, again along the granitoid-Newland contact, contains anomalous abundances of arsenic, cadmium, and molybdenum. The Little Camas Creek drainage along this same contact contains several areas that have been extensively placered.

In contrast, mineralized rock appears to be much less abundant around the edges of the quartz monzodiorite of Mount Edith. This may be due in part to extremely poor exposure in the area underlain by this pluton. Prospects on the south flank of Mount Edith (fig. 1) are clearly related to small intrusions satellitic to the quartz monzodiorite of Mount Edith. Gossany iron oxide- and hydroxide-rich material from the dump adjacent to one of these prospects contains anomalous abundances of silver, arsenic, gold, bismuth, copper, and molybdenum. The Newland Formation along the edges of these small intrusions is altered to calc-silicate hornfels.

Sahinen (1962) described two fluorite occurrences in the central Big Belt Mountains. The first of these (prospect symbol, fig. 1, near sample site No. 202854), on the northeast ridge of Boulder Mountain, consists of a number of small, dark-purple fluorite veins associated with a dike intruding the biotite granodiorite phase of the Boulder pluton. The 1-4-cm-wide veins form a northwest-trending, steeply dipping zone not more than $20 \mathrm{~cm}$ wide. Sahinen (1962) reported numerous prospect pits in this area; assays of fluorite-bearing samples from the prospect pits contain trace amounts of gold. The second occurrence (prospect symbol, fig. 1, near sample site No. 202850), the Normandy fluorite prospect, is about $5 \mathrm{~km}$ along the ridge extending east-southeast from Boulder Mountain. It consists of fracturefilled veins of fluorite-quartz-limonite in the outermost phase of the Boulder pluton. Veins in this area also strike northwest and dip steeply. Neither of these fluorite occurrences is of sufficient size or grade to constitute a resource.

Lang and others (2000) have described a class of globally dispersed intrusion-related gold systems in which the associated magmatic activity is located well-inboard of subduction zones. Many of the characteristics of this class of plutonic rocks and their associated mineral deposits are similar to those of the Big Belt Mountains intrusive rocks and their associated metal deposits. In particular, anomalous metal abundances, including combinations of arsenic, silver, gold, bismuth, cadmium, copper, molybdenum, and lead, are common to globally dispersed intrusion-related gold systems and to mineralized rock associated with quartz monzodiorite intrusions present in the Big Belt Mountains. In the compilation of intrusion-related gold systems by Lang and others (2000), Miller Mountain is one of the cited examples; but very little information concerning either the geology of Miller Mountain or the larger, associated intrusive suite immediately to its south is presented. The basic framework data are presented here.

\section{Geochronology}

Eight mineral separates from five samples of plutons in the Big Belt Mountains were analyzed by the ${ }^{40} \mathrm{Ar} /{ }^{39} \mathrm{Ar}$ incremental heating technique. Three samples, 202879 (potassium feldspar), 202852 (hornblende and potassium feldspar), and 202853 (biotite and potassium feldspar) represent the outer, intermediate, and core zones of the intrusive suite of Boulder Baldy; sample 202878 (hornblende and potassium feldspar) represents the quartz monzodiorite of Mount Edith; and sample 202883 (potassium feldspar) represents the mineralized stock centered on Miller Mountain. Samples were crushed and sieved and mineral concentrates were prepared using standard gravimetric (heavy liquid) and magnetic techniques. Mineral separates were further purified by handpicking. Separates were sealed in silica vials and irradiated in the central thimble of the U.S. Geological Survey TRIGA reactor for 30 hours. Neutron fluence during irradiation was monitored using hornblende standard Mmhb-1, which has a K-Ar age of 520.4 $\pm 1.7 \mathrm{Ma}$ (Alexander and others, 1978).

After irradiation, the samples were progressively degassed in a double-vacuum resistance furnace in a series of 20-minutelong heating steps to a maximum temperature of $1,450^{\circ} \mathrm{C}$. Apparent ages were calculated using decay constants of Steiger and Jäger (1977). The determination of whether the apparent ages yielded a "plateau" was made using the critical value test of Dalrymple and Lanphere (1969) following the plateau determination of Fleck and others (1977). Only one plateau (for hornblende from sample 202852) was achieved; isochron and weight-average dates are used as best estimates of cooling ages for the other hornblende (202878) and biotite (202853) samples. A detailed description of analytical procedures similar to those used in this study appears in Snee and others (1988). ${ }^{40} \mathrm{Ar} /{ }^{39} \mathrm{Ar}$ results are presented as age spectra (fig. 3) and selected isochron diagrams (fig. 4). Detailed analytical data are presented in footnotes of table 2. Hornblende and biotite apparent ages are interpreted to represent cooling of each mineral through its closure temperature for argon diffusion. For these high-level plutons we use $550^{\circ}$ and $300^{\circ} \mathrm{C}$ as argon-closure temperatures for hornblende and biotite, respectively. Potassium feldspar spectra are assumed to represent mixing among several cooling domains (Lovera and others, 1989). We interpret the high-temperature, high-age step of each spectrum to represent the time of argon 


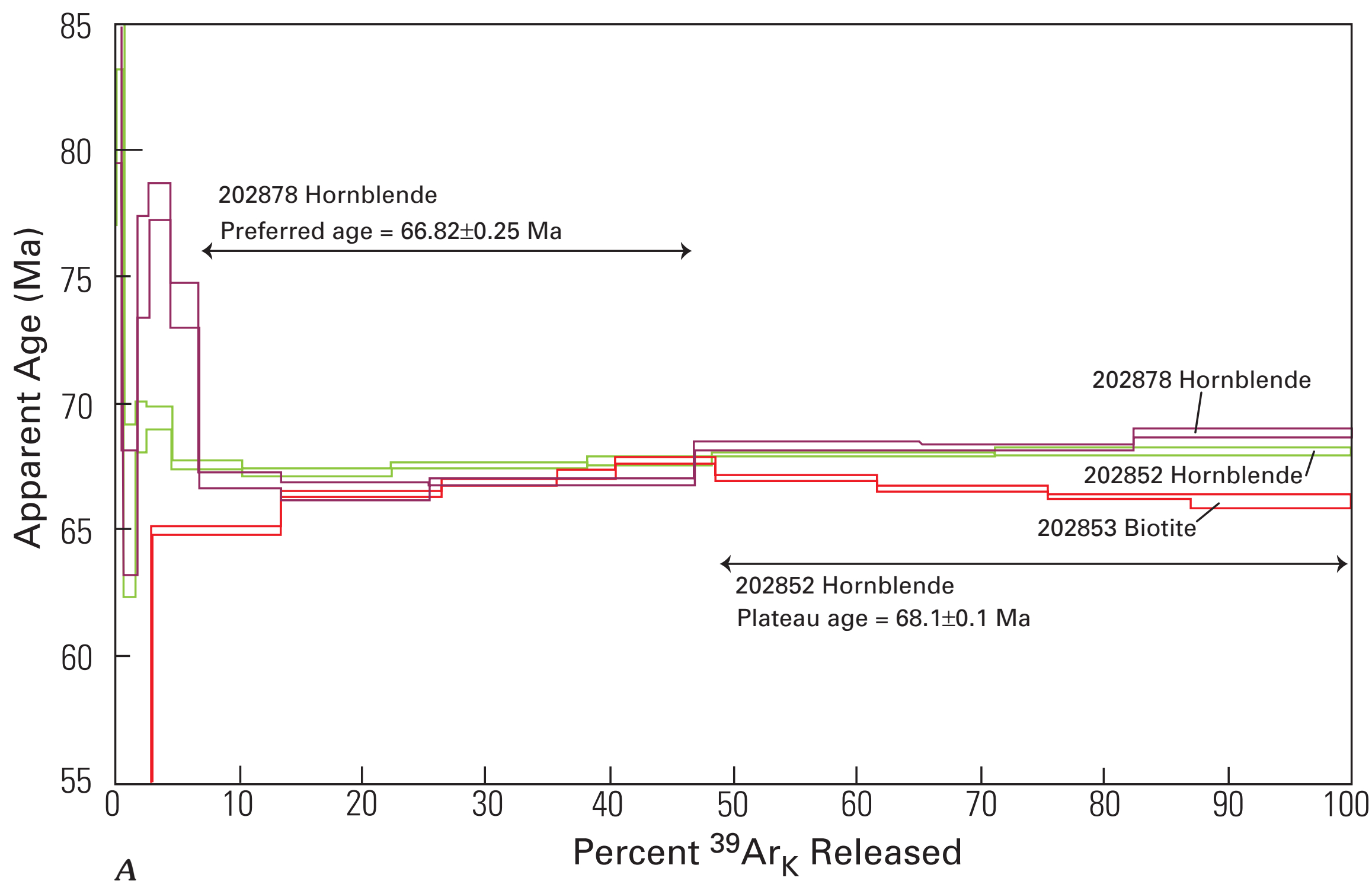

Figure 3 (above and facing page). ${ }^{40} \mathrm{Ar} /{ }^{39} \mathrm{Ar}$ age spectra for separates from intrusions in central Big Belt Mountains, Mont. Vertical width of each bar represents analytical error of each apparent age at $\pm 1 \sigma$. Individual steps used in calculation of plateau dates are indicated. $A$, Spectra for biotite and hornblende separates. $B$, Spectra for potassium feldspar separates. 


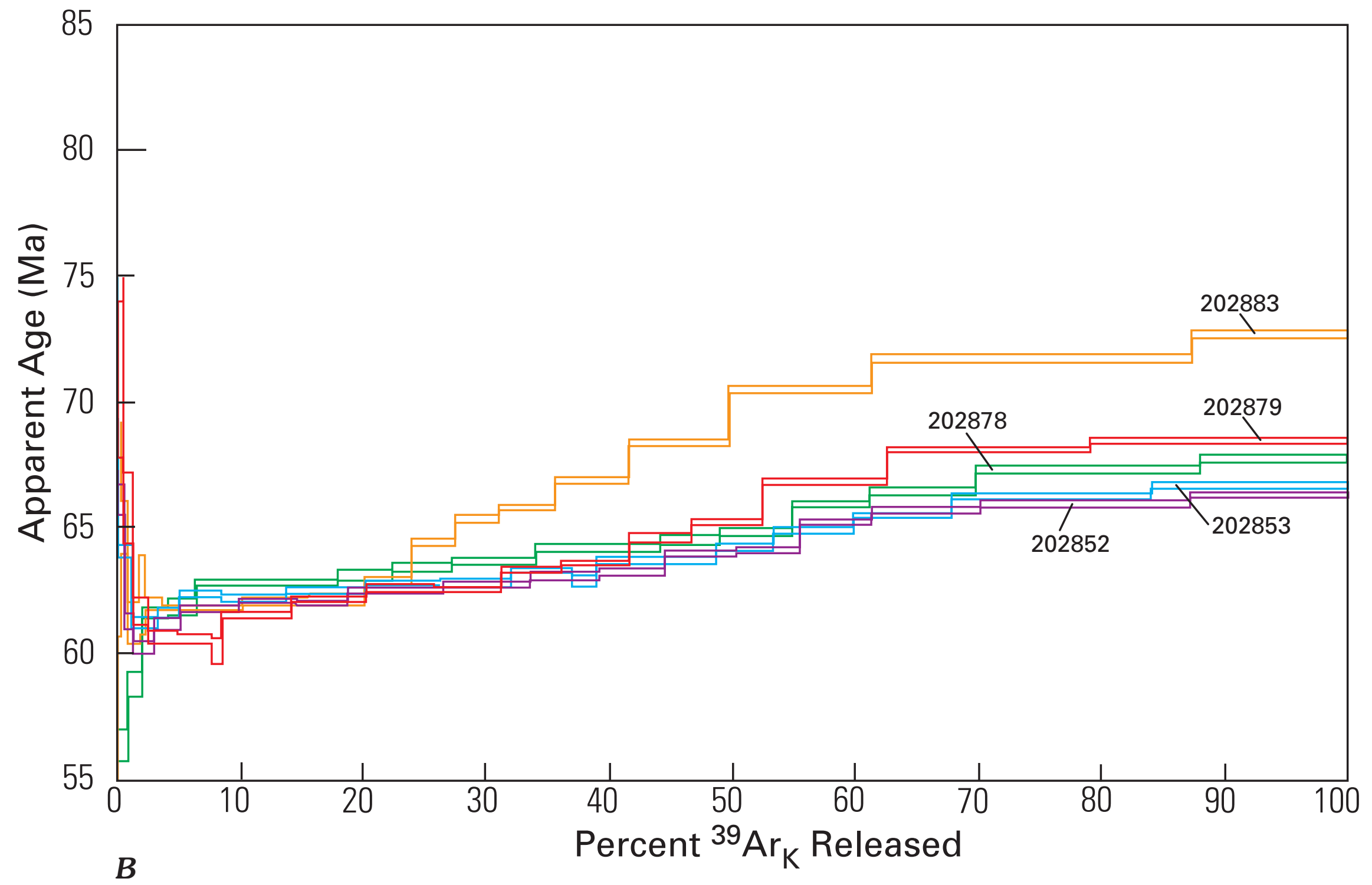



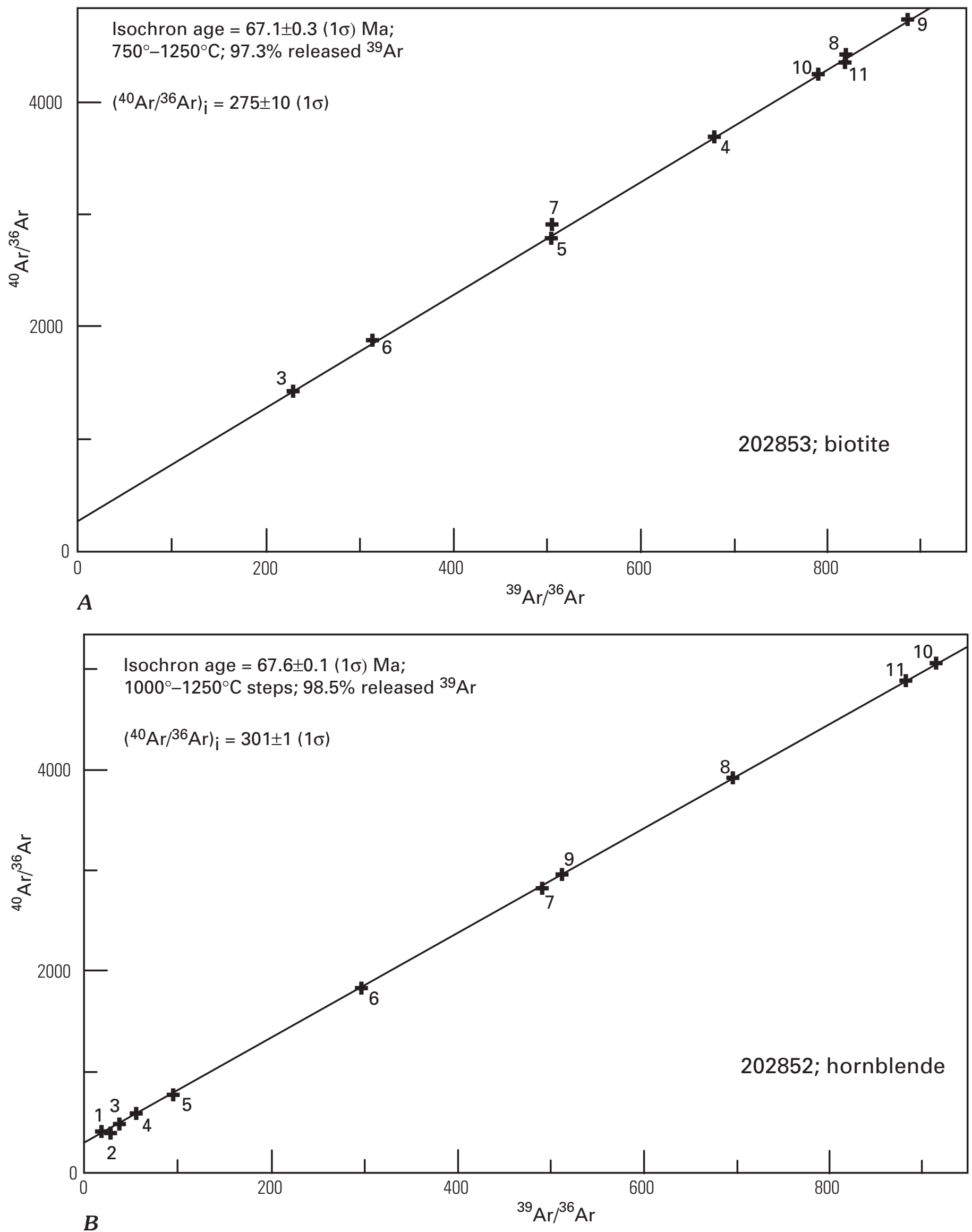

Figure 4 (above and facing page). ${ }^{40} \mathrm{Ar} /{ }^{36} \mathrm{Ar}$ versus ${ }^{39} \mathrm{Ar} /{ }^{36} \mathrm{Ar}$ isotope correlation diagrams for $A, 202853$ biotite, $B, 202852$ hornblende, and $C$, 202878 hornblende. Lines represent linear regressions of data based on method of York (1969). Numbers next to points refer to select temperature steps. 


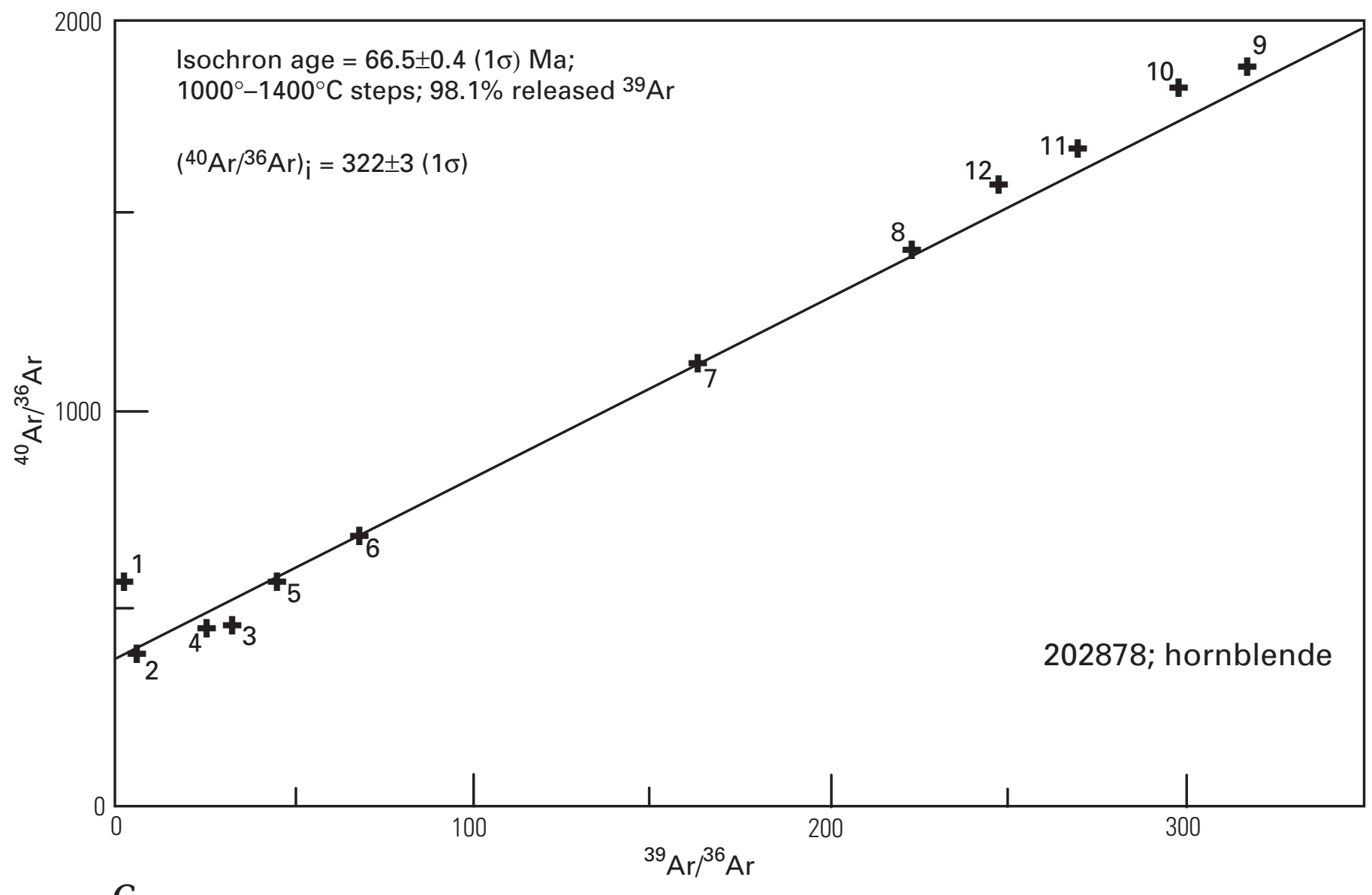

closure of the highest temperature domain. Analogously, the lowest age step or steps represent time of argon closure of the lowest temperature domains. We assume the closure temperature for the lowest temperature cooling domain of the potassium feldspars to be approximately $150^{\circ} \mathrm{C}$.

With these foregoing assumptions, the plateau date of $68.1 \pm 0.1(1 \sigma) \mathrm{Ma}$ for the hornblende from the intermediate zone of the Boulder Baldy pluton represents the time that this part of the pluton cooled through $550^{\circ} \mathrm{C}$. The apparent age of the biotite, $66.5 \pm 0.5 \mathrm{Ma}$, although disturbed, likely represents cooling of the Boulder Baldy pluton's core zone below about $300^{\circ} \mathrm{C}$. Assuming a coupled system, cooling from $550^{\circ}$ to $300^{\circ} \mathrm{C}$ occurred over $1.5 \mathrm{~m} . \mathrm{y}$., yielding a cooling rate of approximately $170^{\circ} \mathrm{C} / \mathrm{m}$.y. Assuming that the Boulder Baldy plutonic suite was emplaced at approximately $750^{\circ} \mathrm{C}$ and at a depth less than 10 $\mathrm{km}$, we infer that some time must have passed between pluton emplacement and its cooling to $550^{\circ} \mathrm{C}$. If the cooling rate from time of emplacement to hornblende argon closure was $170^{\circ} \mathrm{C} /$ m.y. or more, the Boulder Baldy pluton must have been emplaced between 70 and $69 \mathrm{Ma}$. The apparent (isochron date) cooling age $(66.5 \pm 0.4(1 \sigma) \mathrm{Ma})$ for hornblende from the quartz monzodiorite of Mount Edith appears to be statistically younger than quartz monzodiorite in the intrusive suite of Boulder Baldy. If these inferences are accurate, they may reflect a younger emplacement age for the quartz monzodiorite of Mount Edith or a longer period of cooling subsequent to its emplacement. Consequently, plutonic rocks in the central Big Belt Mountains have emplacement and cooling ages similar to the youngest plutons of the nearby Boulder batholith, which is known to have crystallized between 78 and 68 Ma (Tilling, 1973; Snee and others, 1999; Martin and others, 1999).

Apparent ages of potassium feldspar separated from intrusive rocks of the central Big Belt Mountains provide valuable insight into the temporal extent of these intrusives' postmagmatic cooling. The five analyzed potassium feldspars give minimum argon cooling ages derived from lowest temperature extraction steps, of 61.9 to $60.1 \mathrm{Ma}$. The uniformity of these apparent ages, regardless of potassium feldspar compositions, indicates that the region had cooled to relatively low temperatures by $60 \mathrm{Ma}$. We have not experimentally determined closure temperatures for low-temperature cooling domains in the potassium feldspars, but $150^{\circ} \mathrm{C}$ is likely a reasonable estimate. The higher temperature maximum cooling ages for the analyzed potassium feldspars range from 70.7 to $66.1 \mathrm{Ma}$; samples giving the oldest apparent ages were from near the margin of the intrusive suite of Boulder Baldy and from the small, isolated Miller Mountain stock. Again, closure temperatures for the higher temperature cooling domains in the potassium feldspars are unknown, but assuming $300^{\circ}-350^{\circ} \mathrm{C}$, which is consistent with biotite argon closure, these apparent ages are consistent with the general pattern of cooling for the Big Belt Mountains plutonic suite.

The relatively limited difference between the hornblende and biotite ages of the intermediate and core zones of the intrusive suite of Boulder Baldy suggest that magma represented by these samples was part of a single magmatic event. The slightly 
Table 1. Summary of ${ }^{40} \mathrm{Ar} /{ }^{39} \mathrm{Ar}$ age-spectrum results for intrusive rocks from central Big Belt Mountains, Mont.

$[--$, no data $]$

\begin{tabular}{|c|c|c|c|c|c|c|}
\hline Sample & Unit $^{1}$ & Mineral & $\begin{array}{l}\text { Apparent } \\
\text { age (Ma) }\end{array}$ & $\begin{array}{c}\text { Type of } \\
\text { apparent age (Ma) }\end{array}$ & $\begin{array}{l}\text { Correlation } \\
\text { age (Ma) }\end{array}$ & $\begin{array}{l}\text { Initial } \\
40 / 36\end{array}$ \\
\hline \multirow[t]{2}{*}{202879} & \multirow[t]{2}{*}{ bo } & \multirow[t]{2}{*}{$\begin{array}{l}\text { Potassium } \\
\text { feldspar. }\end{array}$} & 68.5 & $\begin{array}{l}\text { Cooling } \\
\text { maximum. }\end{array}$ & -- & -- \\
\hline & & & 60.1 & $\begin{array}{l}\text { Cooling } \\
\text { minimum. }\end{array}$ & -- & -- \\
\hline \multirow[t]{3}{*}{202852} & \multirow[t]{3}{*}{ bi } & Hornblende & $68.1 \pm 0.1$ & Plateau $(51.7 \%)$ & $67.6 \pm 0.1$ & $301 \pm 1$ \\
\hline & & \multirow[t]{2}{*}{$\begin{array}{l}\text { Potassium } \\
\text { feldspar. }\end{array}$} & 66.3 & $\begin{array}{l}\text { Cooling } \\
\text { maximum. }\end{array}$ & -- & -- \\
\hline & & & 60.1 & $\begin{array}{l}\text { Cooling } \\
\text { minimum. }\end{array}$ & -- & -- \\
\hline \multirow[t]{3}{*}{202853} & \multirow[t]{3}{*}{$\mathrm{bc}$} & Biotite & $66.5 \pm 0.5$ & Weight average & $67.1 \pm 0.3$ & $275 \pm 10$ \\
\hline & & \multirow[t]{2}{*}{$\begin{array}{l}\text { Potassium } \\
\text { feldspar. }\end{array}$} & 66.1 & $\begin{array}{l}\text { Cooling } \\
\text { maximum. }\end{array}$ & -- & -- \\
\hline & & & 61.1 & $\begin{array}{l}\text { Cooling } \\
\text { minimum. }\end{array}$ & -- & -- \\
\hline \multirow[t]{3}{*}{202878} & \multirow[t]{3}{*}{$\mathrm{qm}$} & Hornblende & $66.8 \pm 0.2$ & $\begin{array}{c}\text { Excess argon } \\
\text { saddle-low. }\end{array}$ & $66.5 \pm 0.4$ & $322 \pm 3$ \\
\hline & & \multirow[t]{2}{*}{$\begin{array}{l}\text { Potassium } \\
\text { feldspar. }\end{array}$} & 66.8 & $\begin{array}{l}\text { Cooling } \\
\text { maximum. }\end{array}$ & -- & -- \\
\hline & & & 61.1 & $\begin{array}{l}\text { Cooling } \\
\text { minimum. }\end{array}$ & -- & -- \\
\hline \multirow[t]{2}{*}{202883} & \multirow[t]{2}{*}{ MM } & \multirow[t]{2}{*}{$\begin{array}{l}\text { Potassium } \\
\text { feldspar. }\end{array}$} & 70.7 & $\begin{array}{l}\text { Cooling } \\
\text { maximum. } \\
\text { (excess argon?) }\end{array}$ & -- & -- \\
\hline & & & 61.9 & $\begin{array}{l}\text { Cooling } \\
\text { minimum. }\end{array}$ & -- & -- \\
\hline
\end{tabular}

\footnotetext{
${ }^{1}$ bo, bi, bc are outer, intermediate, and core zones of the intrusive suite of Boulder Baldy, respectively; qm, quartz monzodiorite of Mount Edith; MM, mineralized stock at Miller Mountain.
}

older age for hornblende from the suite's intermediate zone is also consistent with the process of margins-inward cooling, through conductive heat loss, that is commonly proposed for solidification of magma chambers to form plutons. The similarity of ages for the quartz monzodiorite of Mount Edith and for the intrusive suite of Boulder Baldy suggests that these masses too are part of a single magmatic event. Finally, the similarity between the age for potassium feldspar from the small, mineralized intrusion exposed at Miller Mountain and those for the intrusive suite of Boulder Baldy and the quartz monzodiorite of Mount Edith indicates that the hydrothermal processes responsible for mineralization at Miller Mountain are temporally and therefore probably genetically related to igneous activity in this area.

\section{Whole-Rock Chemistry}

Compositional variation is documented by analytical data for 49 samples (fig. 1; table 3) of the central Big Belt Mountains intrusions. A representative subset of 14 samples was selected for instrumental neutron activation analysis (table 4). All geochemical analyses were performed in analytical laboratories of the U.S. Geological Survey in Denver, Colo. Major oxide analyses were performed (analysts, D.F. Siems and J.S. Mee) using X-ray fluorescence techniques (Taggart and others, 1987). $\mathrm{Fe}^{2+}$ : total iron as $\mathrm{Fe}^{2+}$ ratios were adjusted to 0.90 and major oxide abundances recalculated to 100 percent, volatile free. $\mathrm{Rb}$, $\mathrm{Sr}, \mathrm{Y}, \mathrm{Zr}, \mathrm{Nb}$, and $\mathrm{Ba}$ abundances were determined (analyst, E.A. du Bray) by energy-dispersive X-ray fluorescence spectroscopy (Elsass and du Bray, 1982; Yager and Quick, 1992) using ${ }^{109} \mathrm{Cd}$ and ${ }^{241} \mathrm{Am}$ radio-isotope excitation sources. Instrumental neutron activation analyses (table 4) were conducted (analyst, J.N. Grossman) using techniques described by Baedecker and McKown (1987).

\section{Major Oxide Data}

Cretaceous intrusive rocks in the central Big Belt Mountains contain between about 60 and almost 75 weight percent $\mathrm{SiO}_{2}$ (table 3); compositional variation of quartz monzodiorite and granodiorite within this range is fairly smooth and essentially continuous (fig. 5). The composition of the quartz monzodiorite of Mount Edith and that of the intrusive suite of Boulder Baldy are overlapping and span most of this composition range. Quartz monzonite and quartz monzodiorite compositions overlap, in part. The granodiorite composition, however, is distinct 
Table 2. ${ }^{40} \mathrm{Ar} /{ }^{39} \mathrm{Ar}$ incremental heating data for samples ${ }^{1}$ from central Big Belt Mountains.

\begin{tabular}{|c|c|c|c|c|c|c|c|}
\hline $\begin{array}{l}\text { Temp } \\
\left({ }^{\circ} \mathrm{C}\right)\end{array}$ & ${ }^{40} \mathrm{Ar}_{\mathrm{R}}{ }^{2}$ & ${ }^{39} \mathrm{Ar}_{\mathrm{K}}{ }^{2}$ & ${ }^{40} \mathrm{Ar}_{\mathrm{R}} /{ }^{39} \mathrm{Ar}_{\mathrm{K}}{ }^{2}$ & ${ }^{39} \mathrm{Ar} /{ }^{37} \mathrm{Ar}^{2,3}$ & ${ }^{0}{ }^{40} \mathrm{Ar}_{\mathrm{R}}$ & $\%{ }^{39} \mathrm{Ar}$ & $\begin{array}{l}\text { Apparent age } e^{4,5} \\
(\text { Ma at } \pm 1 \sigma)\end{array}$ \\
\hline \multicolumn{8}{|c|}{$\begin{array}{l}202879 \text { (Intrusive suite of Boulder Baldy, outer zone) } \\
\text { Potassium feldspar; } 54.3 \mathrm{mg} \text {; J-value }{ }^{5}=0.007215 \pm 0.1 \%(1 \sigma)\end{array}$} \\
\hline 500 & 0.2000 & 0.00757 & 26.4 & 223 & 71.1 & 0.1 & $315 . \pm 7$ \\
\hline 550 & .1800 & .02291 & 7.856 & 441 & 74.7 & .3 & $99.5 \pm 0.9$ \\
\hline 600 & .2712 & .05267 & 5.149 & 487 & 88.3 & .8 & $66 . \pm 1$ \\
\hline 650 & .4042 & .08385 & 4.822 & 240 & 92.4 & 1.2 & $61.7 \pm .6$ \\
\hline 700 & .73494 & .15517 & 4.736 & 471 & 94.1 & 2.3 & $60.62 \pm .22$ \\
\hline 750 & .90530 & .19125 & 4.734 & 329 & 78.1 & 2.8 & $60.58 \pm .19$ \\
\hline 800 & .2720 & .05790 & 4.697 & 341 & 89.8 & .9 & $60.1 \pm .6$ \\
\hline 850 & 1.8393 & .38231 & 4.811 & 348 & 96.6 & 5.6 & $61.56 \pm .09$ \\
\hline 900 & 2.0147 & .41448 & 4.861 & 359 & 97.2 & 6.1 & $62.18 \pm .10$ \\
\hline 950 & 1.8398 & .37607 & 4.892 & 536 & 97.9 & 5.5 & $62.58 \pm .12$ \\
\hline 1000 & 1.8131 & .37083 & 4.889 & 620 & 97.1 & 5.5 & $62.54 \pm .10$ \\
\hline 1050 & 1.6475 & .33260 & 4.954 & 596 & 97.1 & 4.9 & $63.35 \pm .11$ \\
\hline 1100 & 1.8617 & .37421 & 4.975 & 562 & 96.0 & 5.5 & $63.62 \pm .10$ \\
\hline 1150 & 1.7247 & .34124 & 5.054 & 508 & 96.1 & 5.0 & $64.61 \pm .17$ \\
\hline 1200 & 2.0056 & .39291 & 5.105 & 331 & 96.0 & 5.8 & $65.25 \pm .11$ \\
\hline 1250 & 3.5970 & .68769 & 5.231 & 836 & 95.4 & 10.1 & $66.83 \pm .10$ \\
\hline 1300 & 5.9777 & 1.1208 & 5.333 & 54015 & 95.3 & 16.5 & $68.12 \pm .10$ \\
\hline 1400 & 7.6344 & 1.4239 & 5.362 & 1506 & 94.3 & 21.0 & $68.47 \pm .10$ \\
\hline \multicolumn{8}{|c|}{$\begin{array}{l}\text { Total gas date: } 65.75 \pm 0.13 \mathrm{Ma} \\
\text { No plateau }\end{array}$} \\
\hline \multicolumn{8}{|c|}{$\begin{array}{l}202852 \text { (Intrusive suite of Boulder Baldy, intermediate zone) } \\
\text { Hornblende; } 234 \mathrm{mg} ; \mathrm{J} \text {-value } \mathrm{e}^{5}=0.007356 \pm 0.1 \%(1 \sigma)\end{array}$} \\
\hline 650 & 0.0894 & 0.00325 & 27.5 & .29 & 10.4 & 0.1 & 332. \pm 8 \\
\hline 800 & .0952 & .01327 & 7.17 & .90 & 8.0 & .5 & 93. \pm 2 \\
\hline 950 & .1103 & .02187 & 5.04 & .75 & 31.0 & .9 & 66. \pm 3 \\
\hline 1000 & .1235 & .02325 & 5.310 & .28 & 44.8 & .9 & $69.1 \pm 1$ \\
\hline 1050 & .2732 & .05122 & 5.334 & .21 & 59.3 & 2.1 & $69.4 \pm .5$ \\
\hline 1075 & .73562 & .14181 & 5.187 & .19 & 83.6 & 5.8 & $67.56 \pm .17$ \\
\hline 1100 & 1.5205 & .29423 & 5.168 & .19 & 89.4 & 12.0 & $67.31 \pm .14$ \\
\hline 1125 & 2.0200 & .38940 & 5.188 & .20 & 92.3 & 15.8 & $67.56 \pm .10$ \\
\hline 1150 & 1.2937 & .24870 & 5.202 & .20 & 89.8 & 10.1 & $67.74 \pm .15$ \\
\hline 1200 & 2.9399 & .56290 & 5.223 & .20 & 94.0 & 22.9 & $68.01 \pm .10$ \\
\hline 1350 & 3.7046 & .70760 & 5.236 & .20 & 93.8 & 28.8 & $68.17 \pm .14$ \\
\hline \multicolumn{8}{|c|}{$\begin{array}{l}\text { Total gas date: } 68.1 \pm 0.1 \mathrm{Ma} \\
\text { Plateau date (steps 10-11): } 68.1 \pm 0.1 \mathrm{Ma} \text {; Isochron date (steps 4-11): } 67.6 \pm 0.1 \mathrm{Ma} ;\left({ }^{40} \mathrm{Ar} /{ }^{36} \mathrm{Ar}\right)_{\mathrm{i}}=301 \pm 1\end{array}$} \\
\hline
\end{tabular}

202852 (Intrusive suite of Boulder Baidy intermediate zone) Potassium feldspar; $65.534 \mathrm{mg}$; J-value ${ }^{5}=0.00727 \pm 0.1 \%(1 \sigma)$

$\begin{array}{rrrrrrrrr}500 & 0.04333 & 0.00433 & 10.017 & 8.1 & 16.1 & 0.1 & 126 . \pm \pm . \\ 550 & .20827 & .04060 & 5.130 & 17 & 52.2 & .5 & 65.7 \pm .6 \\ 600 & .29099 & .06131 & 4.746 & 11 & 64.6 & .8 & 60.87 \pm .30 \\ 650 & .60455 & .12965 & 4.663 & 13 & 88.6 & 1.6 & 59.82 \pm .25 \\ 700 & .75405 & .15912 & 4.739 & 18 & 82.4 & 2.0 & 60.77 \pm .23 \\ 750 & 1.8674 & .39011 & 4.787 & 17 & 97.8 & 4.9 & 61.38 \pm .12 \\ 800 & 1.8199 & .37822 & 4.812 & 18 & 98.2 & 4.8 & 61.69 \pm .09 \\ 850 & 1.5992 & .33294 & 4.803 & 21 & 92.5 & 4.2 & 61.59 \pm .09 \\ 900 & 2.9771 & .61436 & 4.846 & 29 & 95.8 & 7.7 & 62.12 \pm .10 \\ 950 & 2.7501 & .56555 & 4.863 & 52 & 95.7 & 7.1 & 62.33 \pm .10 \\ 1000 & 2.1951 & .44878 & 4.891 & 73 & 95.0 & 5.6 & 62.70 \pm .12 \\ 1050 & 2.0348 & .41506 & 4.903 & 67 & 95.0 & 5.2 & 62.84 \pm .10 \\ 1100 & 2.3330 & .46987 & 4.965 & 44 & 95.6 & 5.9 & 63.63 \pm .10 \\ 1150 & 2.0223 & .40691 & 4.970 & 27 & 93.9 & 5.1 & 63.69 \pm .10 \\ 1200 & 2.3583 & .46612 & 5.059 & 20 & 94.6 & 5.9 & 64.81 \pm .10 \\ 1250 & 3.5779 & .70192 & 5.097 & 26 & 96.0 & 8.8 & 65.29 \pm .10 \\ 1300 & 6.9524 & 1.3589 & 5.116 & 83 & 97.2 & 17.1 & 65.53 \pm .10 \\ 1400 & 5.2371 & 1.0181 & 5.144 & 92 & 96.4 & 12.8 & 65.87 \pm .10\end{array}$


Table 2-Continued. ${ }^{40} \mathrm{Ar} /{ }^{39} \mathrm{Ar}$ incremental heating data for samples ${ }^{1}$ from central Big Belt Mountains.

\begin{tabular}{|c|c|c|c|c|c|c|c|}
\hline $\begin{array}{l}\text { Temp } \\
\left({ }^{\circ} \mathrm{C}\right)\end{array}$ & ${ }^{40} \mathrm{Ar}_{\mathrm{R}}{ }^{2}$ & ${ }^{39} \mathrm{Ar}_{\mathrm{K}}{ }^{2}$ & ${ }^{40} \mathrm{Ar}_{\mathrm{R}} /{ }^{39} \mathrm{Ar}_{\mathrm{K}}{ }^{2}$ & ${ }^{39} \mathrm{Ar} /{ }^{37} \mathrm{Ar}^{2,3}$ & $\%{ }^{40} \mathrm{Ar}_{\mathrm{R}}$ & $\%{ }^{39} \mathrm{Ar}$ & $\begin{array}{l}\text { Apparent age } 4,5 \\
\text { (Ma at } \pm 1 \sigma \text { ) }\end{array}$ \\
\hline \multicolumn{8}{|c|}{$\begin{array}{l}202853 \text { (Intrusive suite of Boulder Baldy, core zone) } \\
\text { Biotite; } 46.2 \mathrm{mg} ; \mathrm{J} \text {-value }=0.007459 \pm 0.1 \%(1 \sigma)\end{array}$} \\
\hline 550 & 0.00353 & 0.00430 & 0.820 & 13 & 2.0 & 0.1 & 11. \pm 11 \\
\hline 650 & .34396 & .11054 & 3.112 & 38 & 43.2 & 2.6 & $41.39 \pm .40$ \\
\hline 750 & 2.1995 & .44783 & 4.912 & 172 & 79.1 & 10.5 & $64.91 \pm .15$ \\
\hline 850 & 2.7721 & .55203 & 5.022 & 226 & 91.8 & 13.0 & $66.34 \pm .10$ \\
\hline 900 & 2.0069 & .39691 & 5.056 & 157 & 89.5 & 9.3 & $66.79 \pm .10$ \\
\hline 950 & 1.0112 & 19899 & 5.082 & 106 & 84.2 & 4.7 & $67.12 \pm .17$ \\
\hline 1000 & 1.7753 & .34626 & 5.127 & 124 & 89.6 & 8.1 & $67.71 \pm .10$ \\
\hline 1050 & 2.8026 & .55287 & 5.069 & 183 & 93.2 & 13.0 & $66.95 \pm .10$ \\
\hline 1100 & 2.9671 & .58904 & 5.037 & 68 & 93.6 & 13.9 & $66.54 \pm .11$ \\
\hline 1150 & 2.4650 & .49148 & 5.016 & 108 & 92.9 & 11.6 & $66.26 \pm .10$ \\
\hline 1250 & 2.7963 & .55901 & 5.002 & 59 & 93.3 & 13.2 & $66.09 \pm .26$ \\
\hline
\end{tabular}

\begin{tabular}{|c|c|c|c|c|c|c|c|}
\hline \multicolumn{8}{|c|}{$\begin{array}{l}202853 \text { (Intrusive suite of Boulder Baldy, core zone) } \\
\text { Potassium feldspar; } 56.7 \mathrm{mg} \text {; J-value }{ }^{5}=0.007415 \pm 0.1 \%(1 \sigma)\end{array}$} \\
\hline 450 & 0.00831 & 0.00030 & 28.0 & 2.6 & 41.8 & 0.0 & $340 . \pm 21$ \\
\hline 500 & .03513 & .00520 & 6.760 & 7.9 & 34.7 & .1 & $88 . \pm 3$ \\
\hline 550 & .05659 & .01429 & 3.961 & 20 & 61.2 & .2 & 55. \pm 3 \\
\hline 650 & .20435 & .04742 & 4.310 & 15 & 87.1 & .7 & $56.8 \pm .54$ \\
\hline 700 & .38151 & .08548 & 4.463 & 14 & 93.2 & 1.2 & $58.73 \pm .42$ \\
\hline 750 & 64913 & .13968 & 4.647 & 21 & 85.0 & 2.0 & $61.12 \pm .16$ \\
\hline 800 & .72731 & .15608 & 4.660 & 20 & 97.1 & 2.3 & $61.28 \pm .25$ \\
\hline 850 & 3.7059 & .78469 & 4.723 & 23 & 98.9 & 11.5 & $62.09 \pm .10$ \\
\hline 900 & 1.3853 & .29234 & 4.739 & 38 & 98.8 & 4.3 & $62.30 \pm .16$ \\
\hline 950 & 1.5843 & .33287 & 4.760 & 43 & 98.9 & 4.9 & $62.57 \pm .10$ \\
\hline 1000 & 2.2355 & .46871 & 4.770 & 60 & 98.6 & 6.8 & $62.70 \pm .10$ \\
\hline 1050 & 3.2704 & 68013 & 4.809 & 73 & 98.8 & 9.9 & $63.20 \pm .10$ \\
\hline 1100 & 1.5864 & .32858 & 4.828 & 54 & 98.5 & 4.8 & $63.45 \pm .15$ \\
\hline 1150 & 1.9413 & .40041 & 4.849 & 32 & 98.3 & 5.8 & $63.72 \pm .10$ \\
\hline 1200 & 2.0673 & .42007 & 4.921 & 23 & 98.3 & 6.1 & $64.66 \pm .10$ \\
\hline 1250 & 2.9231 & .59010 & 4.954 & 31 & 98.0 & 8.6 & $65.08 \pm .12$ \\
\hline 1300 & 6.1905 & 1.2362 & 5.008 & 96 & 98.5 & 18.0 & $65.77 \pm .10$ \\
\hline 1400 & 4.3768 & .86952 & 5.034 & 79 & 97.8 & 12.7 & $66.11 \pm .10$ \\
\hline \multicolumn{8}{|c|}{ Total gas date: $63.92 \pm 0.12 \mathrm{Ma}$} \\
\hline
\end{tabular}

o

No plateau Hornblende; $195.9 \mathrm{mg}$; J-value ${ }^{5}=0.007492 \pm 0.1 \%(1 \sigma)$

$\begin{array}{rrrrrrrrl}650 & 0.15831 & 0.00093 & 170 & .14 & 48.1 & 0.1 & 1486 . & \pm 44 . \\ 700 & .11242 & .00862 & 13 & .73 & 22.0 & .5 & 168 . & \pm 5 . \\ 950 & .10560 & .02133 & 4.950 & .56 & 35.0 & 1.3 & 66 . & \pm 2 . \\ 1000 & .09226 & .01619 & 5.700 & .25 & 33.2 & 1.0 & 75 . & \pm 2 . \\ 1050 & .16758 & .02840 & 5.901 & .18 & 45.8 & 1.7 & 78.0 \pm & .8 \\ 1075 & .21445 & .03843 & 5.580 & .18 & 55.9 & 2.3 & 73.9 \pm .9 \\ 1100 & .56409 & .11175 & 5.048 & .17 & 73.4 & 6.6 & 66.97 \pm .30 \\ 1125 & 1.0039 & .20030 & 5.012 & .16 & 79.0 & 11.9 & 66.50 \pm .33 \\ 1150 & 1.8159 & .35985 & 5.046 & .16 & 84.3 & 21.4 & 66.95 \pm .13 \\ 1200 & 1.6037 & .31111 & 5.155 & .16 & 83.7 & 18.5 & 68.36 \pm .11 \\ 1250 & 1.4797 & .28749 & 5.147 & .16 & 82.3 & 17.1 & 68.26 \pm .10 \\ 1400 & 1.5461 & .29774 & 5.193 & .16 & 81.2 & 17.7 & 68.85 \pm .11\end{array}$

Total gas date: $69.85 \pm 0.24 \mathrm{Ma}$

No plateau; Isochron date (steps 3-11): $66.5 \pm 0.4 \mathrm{Ma} ;\left({ }^{40} \mathrm{Ar} /{ }^{36} \mathrm{Ar}\right)_{\mathrm{i}}=322 \pm 3$ 
Table 2-Continued. ${ }^{40} \mathrm{Ar} /{ }^{39} \mathrm{Ar}$ incremental heating data for samples ${ }^{1}$ from central Big Belt Mountains.

\begin{tabular}{|c|c|c|c|c|c|c|c|}
\hline $\begin{array}{l}\text { Temp } \\
\left({ }^{\circ} \mathrm{C}\right)\end{array}$ & ${ }^{40} \mathrm{Ar}_{\mathrm{R}}{ }^{2}$ & ${ }^{39} \mathrm{Ar}_{\mathrm{K}}{ }^{2}$ & ${ }^{40} \mathrm{Ar}_{\mathrm{R}} /{ }^{39} \mathrm{Ar}_{\mathrm{K}}{ }^{2}$ & ${ }^{39} \mathrm{Ar} /{ }^{37} \mathrm{Ar}^{2,3}$ & ${ }^{40}{ }^{40} r_{R}$ & ${ }^{39} \mathrm{Ar}$ & $\begin{array}{l}\text { Apparent age }{ }^{4,5} \\
(\text { Ma at } \pm 1 \sigma)\end{array}$ \\
\hline \multicolumn{8}{|c|}{$\begin{array}{l}202878 \text { (Quartz monzodiorite of Mount Edith) } \\
\text { Potassium feldspar; } 55.9 \mathrm{mg} \text {; } \mathrm{J} \text {-value } \mathrm{e}^{5}=0.00711 \pm 0.1 \%(1 \sigma)\end{array}$} \\
\hline 500 & 0.08564 & .00678 & 12.6 & 75 & 56.3 & 0.1 & 155. \pm 6 \\
\hline 550 & .46338 & .09130 & 5.075 & 175 & 90.3 & 1.2 & $63.95 \pm .24$ \\
\hline 600 & .76503 & .15787 & 4.846 & 196 & 93.8 & 2.0 & $61.11 \pm .19$ \\
\hline 650 & .67039 & .13734 & 4.881 & 175 & 80.3 & 1.8 & $61.55 \pm .19$ \\
\hline 700 & 1.3384 & .27097 & 4.939 & 133 & 95.0 & 3.5 & $62.27 \pm .14$ \\
\hline 750 & 2.0043 & .40669 & 4.928 & 126 & 97.0 & 5.2 & $62.13 \pm .14$ \\
\hline 800 & 2.5138 & .50728 & 4.956 & 134 & 97.7 & 6.5 & $62.47 \pm .10$ \\
\hline 850 & 2.3266 & .46739 & 4.978 & 150 & 97.7 & 6.0 & $62.74 \pm .10$ \\
\hline 900 & 2.2649 & .45485 & 4.980 & 180 & 97.7 & 5.8 & $62.76 \pm .16$ \\
\hline 950 & 1.9089 & .37996 & 5.024 & 251 & 97.1 & 4.9 & $63.32 \pm .10$ \\
\hline 1000 & .76492 & .15338 & 4.987 & 255 & 94.5 & 2.0 & $62.86 \pm .23$ \\
\hline 1050 & 1.9819 & .39172 & 5.060 & 270 & 98.1 & 5.0 & $63.76 \pm .15$ \\
\hline 1100 & 1.8723 & .36998 & 5.061 & 256 & 97.4 & 4.7 & $63.77 \pm .17$ \\
\hline 1150 & 1.8298 & .35881 & 5.100 & 207 & 97.4 & 4.6 & $64.25 \pm .17$ \\
\hline 1200 & 2.6608 & .51621 & 5.155 & 165 & 97.4 & 6.6 & $64.93 \pm .13$ \\
\hline 1250 & 3.2391 & .62232 & 5.205 & 251 & 97.2 & 8.0 & $65.56 \pm .10$ \\
\hline 1300 & 6.6508 & 1.2626 & 5.267 & 501 & 97.6 & 16.2 & $66.33 \pm .10$ \\
\hline 1400 & 6.5930 & 1.2426 & 5.306 & 499 & 96.8 & 15.9 & $66.80 \pm .10$ \\
\hline \multicolumn{8}{|c|}{$\begin{array}{l}\text { Total gas date: } 64.52 \pm 0.13 \mathrm{Ma} \\
\text { No plateau }\end{array}$} \\
\hline \multicolumn{8}{|c|}{$\begin{array}{l}202883 \text { (Altered quartz monzodiorite at Miller Mountain) } \\
\text { Potassium feldspar; } 71.2 \mathrm{mg} \text {; J-value }{ }^{5}=0.0075421 \pm 0.1 \%(1 \sigma)\end{array}$} \\
\hline 450 & 0.38767 & .01533 & 25.2 & 21 & 78.0 & 0.2 & 315. \pm 3 \\
\hline 500 & .03265 & .00700 & 4.665 & 24 & 51.1 & .1 & 62. \pm 6 \\
\hline 550 & .06844 & .01421 & 4.818 & 30 & 69.7 & .2 & 64. \pm 3 \\
\hline 600 & .21640 & .04487 & 4.822 & 28 & 87.9 & .5 & $64.4 \pm .8$ \\
\hline 650 & .43549 & .09503 & 4.583 & 19 & 90.8 & 1.0 & $61.3 \pm .7$ \\
\hline 700 & .18095 & .03889 & 4.653 & 20 & 94.2 & .4 & 66. \pm 1 \\
\hline 750 & .55873 & .12069 & 4.630 & 24 & 80.2 & 1.3 & $61.91 \pm .18$ \\
\hline 800 & 2.7939 & .60444 & 4.622 & 42 & 93.9 & 6.5 & $61.82 \pm .09$ \\
\hline 850 & 2.2848 & .49293 & 4.635 & 40 & 97.1 & 5.3 & $61.99 \pm .15$ \\
\hline 900 & 1.9324 & .41651 & 4.640 & 41 & 97.4 & 4.5 & $62.05 \pm .19$ \\
\hline 950 & 1.6311 & .34814 & 4.685 & 54 & 97.3 & 3.7 & $62.65 \pm .10$ \\
\hline 1000 & 1.5791 & .33021 & 4.782 & 72 & 97.7 & 3.6 & $63.92 \pm .13$ \\
\hline 1050 & 1.5773 & .32587 & 4.840 & 75 & 97.1 & 3.5 & $64.68 \pm .14$ \\
\hline 1100 & 2.0466 & .42029 & 4.870 & 62 & 96.2 & 4.5 & $65.07 \pm .10$ \\
\hline 1150 & 2.7129 & .54961 & 4.936 & 57 & 96.5 & 5.9 & $65.94 \pm .11$ \\
\hline 1200 & 3.7620 & .74787 & 5.030 & 59 & 96.9 & 8.0 & $67.18 \pm .10$ \\
\hline 1250 & 5.5118 & 1.0673 & 5.164 & 92 & 97.3 & 11.5 & $68.93 \pm .11$ \\
\hline 1300 & 12.533 & 2.3918 & 5.240 & 280 & 97.5 & 25.7 & $69.92 \pm .11$ \\
\hline 1400 & 6.7157 & 1.2672 & 5.300 & 338 & 96.4 & 13.6 & $70.70 \pm .11$ \\
\hline \multicolumn{8}{|c|}{ Total gas date: $67.44 \pm 0.14 \mathrm{Ma}$} \\
\hline
\end{tabular}

${ }^{1}$ Mineral separates were prepared after crushing, grinding, and sieving by magnetic separator, mica-table, and heavyliquid methods; grains ranged in size between 60 and 120 mesh $(250-125 \mu \mathrm{m})$. Separates were handpicked to greater than $99 \%$ purity. Individual samples, which ranged in mass from 46 to $234 \mathrm{mg}$, were cleaned with reagent-grade acetone, alcohol, and deionized water in an ultrasonic bath, air-dried, wrapped in aluminum capsules and sealed in silica vials along with monitor minerals before irradiation. Samples were irradiated at the TRIGA reactor facility at the U.S. Geological Survey in Denver, Colo., in one irradiation package. For irradiation an aluminum canister was loaded with 
Table 2-Continued. ${ }^{40} \mathrm{Ar} /{ }^{39} \mathrm{Ar}$ incremental heating data for samples ${ }^{1}$ from central Big Belt Mountains.

\begin{abstract}
six quartz vials each of which was loaded with samples and standards. Standards were placed between every two unknowns as well as at the top and bottom of each vial. Each standard was degassed to release argon in a single 20minute-long heating step at $1,250^{\circ} \mathrm{C}$. Each sample was degassed stepwise in a series of 11 to 18 individual temperature steps for 20 minutes each. All analyses were done in the Argon Laboratory, U.S. Geological Survey, Denver, Colo. Decay constants are those of Steiger and Jäger (1977). The standard for this experiment is hornblende MMhb-1 with percent $\mathrm{K}=1.555,{ }^{40} \mathrm{ArR}=1.624 \times 10^{-9} \mathrm{~mole} / \mathrm{g}$, and $\mathrm{K}-\mathrm{Ar}$ age $=520.4 \mathrm{Ma}$ (Samson and Alexander, 1987).

2 Abundances of "Radiogenic ${ }^{40} \mathrm{Ar}$ " and "K-derived ${ }^{39} \mathrm{Ar}$ " are reported in volts measured on a Mass Analyser Products 215 rare-gas mass spectrometer using the Faraday cup. Conversion to moles can be made using a sensitivity of $9.736 \times 10^{-13}$ moles argon per volt of signal. Detection limit at the time of this experiment was $2 \times 10^{-17}$ moles argon. Corrections were made for volume, mass fractionation, trap current, radioactive decay of ${ }^{37} \mathrm{Ar}$ and ${ }^{39} \mathrm{Ar}$, and interfering Ar isotopes. Mass discrimination in our mass spectrometer is routinely determined by measuring the ${ }^{40} \mathrm{Ar} /{ }^{36} \mathrm{Ar}$ ratio of atmospheric argon; our measured value is generally 298.9; the accepted value is 295.5 . The calculated discrimination is applied to all samples and standards equally. Production ratios measured on pure $\mathrm{K}_{2} \mathrm{SO}_{4}$ and $\mathrm{CaF}_{2}$ salts irradiated with the samples were used to correct for irradiation-produced ${ }^{40} \mathrm{Ar}$ (from K) and ${ }^{39} \mathrm{Ar}$ (from Ca). Corrections for Cl-derived ${ }^{36} \mathrm{Ar}$ were determined using the method of Roddick (1983). Production ratios determined for samples in this experiment are: $\left({ }^{40} \mathrm{Ar}{ }^{\beta 9} \mathrm{Ar}\right)_{\mathrm{K}}=9.35 \times 10^{-3},\left({ }^{38} \mathrm{Ar} /{ }^{39} \mathrm{Ar}\right)_{\mathrm{K}}=1.304 \times 10^{-2},\left({ }^{37} \mathrm{Ar}{ }^{39} \mathrm{Ar}\right)_{\mathrm{K}}=3.0 \times 10^{-4},\left({ }^{36} \mathrm{Ar} /\right.$ $\left.{ }^{37} \mathrm{Ar}\right)_{\mathrm{Ca}}=2.7 \times 10^{-4},\left({ }^{39} \mathrm{Ar} /{ }^{37} \mathrm{Ar}\right)_{\mathrm{Ca}}=7.2 \times 10^{-4},\left({ }^{38} \mathrm{Ar} /{ }^{37} \mathrm{Ar}\right)_{\mathrm{Ca}}=4.7 \times 10^{-5}$. Analytical data for "Radiogenic ${ }^{40} \mathrm{Ar}$ " and "K-derived ${ }^{39} \mathrm{Ar}$ " are calculated to five places; " ${ }^{40} \mathrm{Ar}_{\mathrm{R}} /{ }^{39} \mathrm{Ar}_{\mathrm{K}}$ " is calculated to three decimal places. "Radiogenic

${ }^{40} \mathrm{Ar}$," "K-derived ${ }^{39} \mathrm{Ar}$," and " ${ }^{40} \mathrm{Ar}_{\mathrm{R}} /{ }^{39} \mathrm{Ar}_{\mathrm{K}}$ " are rounded to significant figures using associated analytical precisions. Apparent ages and associated errors were calculated from unrounded data and then rounded using associated errors.

3 To calculate apparent $\mathrm{K} / \mathrm{Ca}$ ratios, divide ${ }^{39} \mathrm{Ar} /{ }^{37} \mathrm{Ar}$ value by 2 .

${ }^{4}$ Uncertainties in calculations for the apparent date of individual steps for a sample were calculated using the equations of Dalrymple and others (1981).

5 The reproducibility of split gas fractions from selected monitors was used to calculate imprecision in $\mathbf{J}$ values. This imprecision is generally $0.1 \%, 1$ sigma. $\mathrm{J}$ values for each sample were interpolated from adjacent standards. The uncertainty in each apparent age includes the uncertainty in the $\mathrm{J}$ value.
\end{abstract}

and dramatically restricted, as epitomized by $\mathrm{SiO}_{2}$ contents that range from 68.3 to only 70.1 (six samples). The granodiorite contains more $\mathrm{Al}_{2} \mathrm{O}_{3}$ and $\mathrm{CaO}$ and less $\mathrm{Na}_{2} \mathrm{O}$ and $\mathrm{K}_{2} \mathrm{O}$ than other central Big Belt Mountains intrusions with similar $\mathrm{SiO}_{2}$ contents. As is the case for most subalkaline rocks, abundances of $\mathrm{Al}_{2} \mathrm{O}_{3}$, $\mathrm{TiO}_{2}, \mathrm{FeO}^{*}$ (total iron as $\mathrm{FeO}$ ), $\mathrm{MgO}, \mathrm{CaO}, \mathrm{P}_{2} \mathrm{O}_{5}$, and $\mathrm{MnO}$ in the Big Belt intrusions decrease with increasing $\mathrm{SiO}_{2}$ content, whereas abundances of $\mathrm{Na}_{2} \mathrm{O}$ and $\mathrm{K}_{2} \mathrm{O}$ are relatively constant. The mafic border phase of the quartz monzodiorite of Mount Edith is characterized by higher contents of $\mathrm{TiO}_{2}, \mathrm{FeO}^{*}, \mathrm{MgO}$, $\mathrm{CaO}, \mathrm{P}_{2} \mathrm{O}_{5}$, and $\mathrm{MnO}$ and lower contents of $\mathrm{SiO}_{2}, \mathrm{Na}_{2} \mathrm{O}$, and $\mathrm{K}_{2} \mathrm{O}$ than the remainder of this pluton.

The quartz monzonite is characterized by distinctive compositions, which, because they also vary erratically, blur the compositional variation trends just described (fig. 5). The quartz monzonite contains more $\mathrm{Al}_{2} \mathrm{O}_{3}, \mathrm{Na}_{2} \mathrm{O}$, and $\mathrm{K}_{2} \mathrm{O}$ and less $\mathrm{TiO}_{2}$, $\mathrm{FeO}^{*}, \mathrm{MgO}, \mathrm{CaO}, \mathrm{P}_{2} \mathrm{O}_{5}$, and $\mathrm{MnO}$ than other Late Cretaceous igneous rocks of the central Big Belt Mountains. The unusual and erratically variable composition of these rocks, in addition to their mineralogic and textural features, suggests that these are not magmatic rocks. Quartz monzonite samples that best retain primary magmatic textures have compositions that overlap those of the quartz monzodiorite, whereas quartz monzonite samples whose textures indicate the greatest degree of alteration and recrystallization have compositions that stray furthest from those of the quartz monzodiorite. The quartz monzonite samples record a process that caused their compositions to be increasingly perturbed from primary magmatic values.

Most quartz monzodiorite from the central Big Belt Mountains has a total alkali versus silica content in the range between the alkaline-subalkaline dividing lines of Irvine and Baragar (1971) and Le Bas and others (1986); these rocks are transitional from alkaline to subalkaline (fig. 6). In contrast, most of the quartz monzonite is strongly alkaline, whereas the biotite granodiorite is distinctly subalkaline. Most of the quartz monzodiorite compositions plot (fig. 5) in the high-K calc-alkaline field of Ewart (1982); most of the quartz monzonite compositions plot in the shoshonite field, whereas those of the biotite granodiorite and a few of the quartz monzodiorite compositions plot in the calc-alkaline field.

Geochronologic data indicate that ages (Martin and others, 1999; Snee and others, 1999) of plutons in the Boulder batholith, which is across the Missouri River valley from the Big Belt Mountains, are similar to ages (data from the present report) of intrusive rocks of the central Big Belt Mountains. In a study of the Boulder batholith, Tilling (1973) defined two distinct granitic rock composition series, main and sodic, for its constituent plutons. Sodic series rocks are distinguished from those of the main series by slightly higher $\mathrm{Na}_{2} \mathrm{O}$ and $\mathrm{Sr}$ contents and lower $\mathrm{K}_{2} \mathrm{O}$ and $\mathrm{Rb}$ contents at a given $\mathrm{SiO}_{2}$ abundance; ternary proportions of $\mathrm{Na}_{2} \mathrm{O}, \mathrm{K}_{2} \mathrm{O}$, and $\mathrm{CaO}$ for the two series are particularly diagnostic. Compositions of central Big Belt Mountains intrusions (fig. 7) are most like those of the sodic series plutons of Tilling (1973); however, at a given $\mathrm{SiO}_{2}$ content, the Big Belt intrusions contain higher abundances of $\mathrm{Al}_{2} \mathrm{O}_{3}$ and $\mathrm{Na}_{2} \mathrm{O}$ and lower abundances of $\mathrm{FeO}^{*}, \mathrm{MgO}$, and $\mathrm{P}_{2} \mathrm{O}_{5}$ than the sodic series plutons.

\section{Trace-Element Data}

The extent of trace-element abundance variation in intrusions of the central Big Belt Mountains is moderate (fig. 8). Abundances of $\mathrm{Sr}, \mathrm{Co}, \mathrm{Cr}, \mathrm{Sc}, \mathrm{Nb}, \mathrm{Zr}, \mathrm{U}$, and total REE decrease with increasing $\mathrm{SiO}_{2}$, whereas those of $\mathrm{Zn}, \mathrm{Ba}$, Th, 

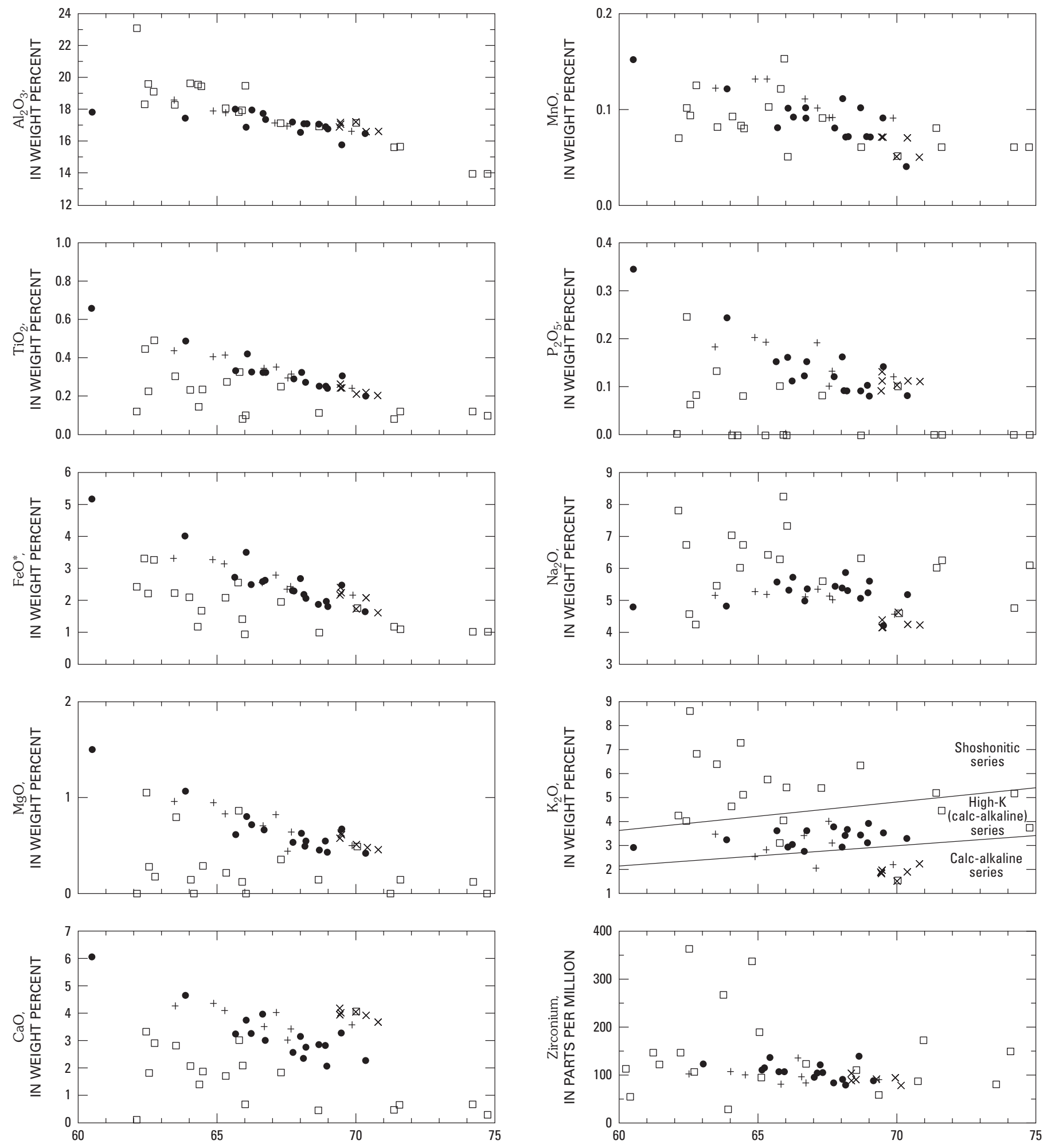

$\mathrm{SiO}_{2}$, IN W'EIGHT PERCENT

\section{EXPLANATION}

Intrusive suite of Boulder Baldy

$\square \quad$ bo, outer zone

+ bi, intermediate: zone

$\times \quad$ bc, core zone

- Quartz monzodiorite of Mount Edith

Figure 5. Variation diagrams showing abundances of major oxides and zirconium in intrusions in central Big Belt Mountains. Discriminant line

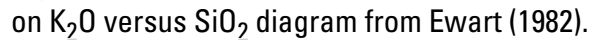


Table 3. Composition of Cretaceous intrusive rocks from central Big Belt Mountains.

[Note: bo, bi, bc, outer, intermediate, and core zones of intrusive suite of Boulder Baldy, respectively; qm, quartz monzodiorite of Mount Edith. Major oxide data in weight percent, trace-element data in parts per million. Ferrous iron/total iron as $\mathrm{FeO}$ adjusted to 0.9; LOI, loss on ignition; ND, not detected]

\begin{tabular}{|c|c|c|c|c|c|c|c|c|c|c|c|c|c|c|c|c|}
\hline $\begin{array}{l}\text { Unit } \\
\text { Sample }\end{array}$ & $\begin{array}{r}\text { bo } \\
202850\end{array}$ & $\begin{array}{r}\text { bo } \\
202851\end{array}$ & $\begin{array}{r}\text { bo } \\
202858\end{array}$ & $\begin{array}{r}\text { bo } \\
202864\end{array}$ & $\begin{array}{r}\text { bo } \\
202865\end{array}$ & $\begin{array}{r}\text { bo } \\
202867\end{array}$ & $\begin{array}{r}\text { bo } \\
202869\end{array}$ & $\begin{array}{r}\text { bo } \\
202876\end{array}$ & $\begin{array}{r}\text { bo } \\
202879\end{array}$ & $\begin{array}{r}\text { bo } \\
202881\end{array}$ & $\begin{array}{r}\text { bo } \\
202882\end{array}$ & $\begin{array}{r}\text { bo } \\
202932\end{array}$ & $\begin{array}{r}\text { bo } \\
202933\end{array}$ & $\begin{array}{r}\text { bo } \\
202945\end{array}$ & $\begin{array}{r}\text { bo } \\
202948\end{array}$ & $\begin{array}{r}\text { bo } \\
202949\end{array}$ \\
\hline $\mathrm{SiO}_{2}$ & 65.10 & 70.70 & 62.20 & 64.70 & 62.70 & 70.90 & 63.90 & 61.20 & 73.50 & 60.20 & 74.00 & 60.40 & 62.50 & 65.00 & 61.40 & 63.70 \\
\hline $\mathrm{Al}_{2} \mathrm{O}_{3}$ & 19.20 & 15.50 & 17.90 & 17.60 & 19.20 & 15.50 & 17.70 & 17.90 & 13.80 & 18.30 & 13.80 & 18.90 & 19.00 & 17.60 & 22.80 & 19.20 \\
\hline $\mathrm{Fe}_{2} \mathrm{O}_{3}$ & 0.20 & 0.26 & 0.49 & 0.31 & 0.46 & 0.24 & 0.45 & 0.72 & 0.22 & 0.69 & 0.23 & 0.48 & 0.25 & 0.56 & 0.53 & 0.37 \\
\hline $\mathrm{FeO}$ & 0.73 & 0.92 & 1.76 & 1.11 & 1.65 & 0.86 & 1.62 & 2.59 & 0.80 & 2.50 & 0.81 & 1.72 & 0.90 & 2.02 & 1.91 & 1.32 \\
\hline $\mathrm{MgO}$ & ND & ND & 0.78 & 0.12 & 0.14 & 0.14 & 0.21 & 1.03 & 0.12 & 0.17 & ND & 0.27 & ND & 0.85 & ND & 0.28 \\
\hline $\mathrm{CaO}$ & 0.68 & 0.48 & 2.76 & 2.05 & 2.03 & 0.64 & 1.67 & 3.25 & 0.66 & 2.80 & 0.28 & 1.77 & 1.35 & 2.97 & 0.11 & 1.85 \\
\hline $\mathrm{Na}_{2} \mathrm{O}$ & 7.20 & 5.95 & 5.32 & 8.08 & 6.87 & 6.18 & 6.28 & 6.60 & 4.70 & 4.07 & 6.03 & 4.40 & 5.84 & 6.20 & 7.70 & 6.64 \\
\hline $\mathrm{K}_{2} \mathrm{O}$ & 5.31 & 5.09 & 6.23 & 3.98 & 4.53 & 4.40 & 5.61 & 3.96 & 5.07 & 6.54 & 3.71 & 8.26 & 7.05 & 3.05 & 4.18 & 5.04 \\
\hline $\mathrm{TiO}_{2}$ & 0.10 & 0.08 & 0.30 & 0.08 & 0.23 & 0.12 & 0.27 & 0.44 & 0.12 & 0.47 & 0.10 & 0.22 & 0.14 & 0.32 & 0.12 & 0.23 \\
\hline $\mathrm{P}_{2} \mathrm{O}_{5}$ & ND & ND & 0.13 & ND & ND & ND & ND & 0.24 & ND & 0.08 & ND & 0.06 & ND & 0.10 & ND & 0.08 \\
\hline $\mathrm{MnO}$ & 0.05 & 0.08 & 0.08 & 0.15 & 0.09 & 0.06 & 0.10 & 0.10 & 0.06 & 0.12 & 0.06 & 0.09 & 0.08 & 0.12 & 0.07 & 0.08 \\
\hline LOI & 0.36 & 0.26 & 0.36 & 0.34 & 0.21 & 0.16 & 0.65 & 0.14 & 0.11 & 0.46 & 0.07 & 1.55 & 1.23 & 0.26 & 1.10 & 0.30 \\
\hline TOTAL & 98.94 & 99.32 & 98.30 & 98.52 & 98.11 & 99.20 & 98.46 & 98.17 & 99.16 & 96.40 & 99.09 & 98.12 & 98.34 & 99.05 & 99.92 & 99.09 \\
\hline $\mathrm{Rb}$ & 99 & 132 & 121 & 97 & 86 & 99 & 124 & 71 & 138 & 100 & 92 & 149 & 112 & 70 & 108 & 87 \\
\hline $\mathrm{Sr}$ & 1395 & 228 & 2209 & 39 & 2659 & 372 & 1166 & 2307 & 315 & 3704 & 50 & 2349 & 1322 & 1430 & 200 & 1118 \\
\hline $\mathrm{Y}$ & 6 & 13 & 21 & 8 & 14 & 13 & 15 & 27 & 5 & 39 & 5 & 10 & 12 & 23 & 6 & 22 \\
\hline $\mathrm{Zr}$ & 94 & 87 & 145 & 335 & 105 & 171 & 27 & 146 & 80 & 113 & 147 & 54 & 360 & 188 & 120 & 267 \\
\hline $\mathrm{Nb}$ & 11 & 16 & 26 & 12 & 48 & 16 & 16 & 26 & 11 & 37 & 16 & 22 & 34 & 17 & 24 & 24 \\
\hline $\mathrm{Ba}$ & 1879 & 766 & 2635 & 79 & 3075 & 808 & 2367 & 3043 & 662 & 6253 & 88 & 4680 & 2661 & 1505 & 479 & 1881 \\
\hline $\mathrm{La}$ & 15 & 9 & 33 & ND & 109 & 6 & 36 & 48 & 8 & 88 & 42 & 73 & 29 & 32 & 67 & 83 \\
\hline $\mathrm{Ce}$ & 18 & 23 & 28 & 4 & 104 & 30 & 61 & 79 & 21 & 60 & 45 & 85 & 55 & 57 & 112 & 102 \\
\hline $\mathrm{Nd}$ & ND & 2 & 33 & ND & 43 & 6 & 28 & 62 & 6 & 81 & 20 & 26 & 22 & 22 & 2 & 47 \\
\hline Unit & bo & bo & bo & bi & bi & $\mathrm{bi}$ & bi & bi & $\mathrm{bi}$ & $\mathrm{bi}$ & $b c$ & $b c$ & $b c$ & $b c$ & $b c$ & $b c$ \\
\hline Sample & 202953 & 202955 & 202957 & 202852 & 202934 & 202944 & 202947 & 202951 & 202954 & 202956 & 202853 & 202854 & 202935 & 202943 & 202946 & 202952 \\
\hline $\mathrm{SiO}_{2}$ & 69.30 & 66.70 & 68.50 & 66.70 & 62.50 & 64.00 & 69.30 & 65.80 & 66.50 & 64.50 & 68.30 & 68.30 & 68.50 & 70.10 & 69.90 & 69.20 \\
\hline $\mathrm{Al}_{2} \mathrm{O}_{3}$ & 17.00 & 17.00 & 16.90 & 16.90 & 18.30 & 17.70 & 16.50 & 17.20 & 17.00 & 17.60 & 16.80 & 16.90 & 16.70 & 16.50 & 16.50 & 17.00 \\
\hline $\mathrm{Fe}_{2} \mathrm{O}_{3}$ & 0.38 & 0.43 & 0.22 & 0.53 & 0.73 & 0.72 & 0.48 & 0.56 & 0.62 & 0.69 & 0.49 & 0.48 & 0.52 & 0.35 & 0.46 & 0.38 \\
\hline $\mathrm{FeO}$ & 1.38 & 1.55 & 0.80 & 1.91 & 2.61 & 2.58 & 1.71 & 2.03 & 2.22 & 2.48 & 1.76 & 1.72 & 1.87 & 1.27 & 1.64 & 1.37 \\
\hline $\mathrm{MgO}$ & 0.48 & 0.35 & 0.14 & 0.63 & 0.94 & 0.93 & 0.50 & 0.67 & 0.81 & 0.82 & 0.60 & 0.57 & 0.62 & 0.45 & 0.47 & 0.50 \\
\hline $\mathrm{CaO}$ & 4.00 & 1.81 & 0.44 & 3.40 & 4.21 & 4.31 & 3.55 & 3.47 & 4.00 & 4.06 & 3.95 & 4.11 & 3.89 & 3.65 & 3.89 & 3.99 \\
\hline $\mathrm{Na}_{2} \mathrm{O}$ & 4.54 & 5.53 & 6.27 & 4.94 & 5.08 & 5.21 & 4.54 & 5.02 & 5.29 & 5.11 & 4.10 & 4.08 & 4.32 & 4.18 & 4.21 & 4.57 \\
\hline $\mathrm{K}_{2} \mathrm{O}$ & 1.52 & 5.33 & 6.31 & 3.07 & 3.41 & 2.50 & 2.18 & 3.37 & 2.04 & 2.81 & 1.91 & 1.83 & 1.81 & 2.19 & 1.88 & 1.51 \\
\hline $\mathrm{TiO}_{2}$ & 0.22 & 0.25 & 0.11 & 0.31 & 0.43 & 0.40 & 0.24 & 0.33 & 0.35 & 0.41 & 0.24 & 0.24 & 0.26 & 0.20 & 0.22 & 0.21 \\
\hline $\mathrm{P}_{2} \mathrm{O}_{5}$ & 0.10 & 0.08 & ND & 0.13 & 0.18 & 0.20 & 0.12 & 0.15 & 0.19 & 0.19 & 0.11 & 0.09 & 0.13 & 0.11 & 0.11 & 0.10 \\
\hline $\mathrm{MnO}$ & 0.05 & 0.09 & 0.06 & 0.09 & 0.12 & 0.13 & 0.09 & 0.11 & 0.10 & 0.13 & 0.07 & 0.07 & 0.07 & 0.05 & 0.07 & 0.05 \\
\hline LOI & 0.34 & 0.30 & 0.15 & 0.27 & 0.26 & 0.29 & 0.34 & 0.21 & 0.23 & 0.36 & 0.50 & 0.49 & 0.45 & 0.32 & 0.30 & 0.37 \\
\hline TOTAL & 99.32 & 99.43 & 99.90 & 98.89 & 98.77 & 98.97 & 99.55 & 98.92 & 99.34 & 99.16 & 98.83 & 98.88 & 99.14 & 99.38 & 99.65 & 99.26 \\
\hline
\end{tabular}




\begin{tabular}{|c|c|c|c|c|c|c|c|c|c|c|c|c|c|c|c|c|c|}
\hline $\mathrm{Rb}$ & 98 & 106 & 97 & 96 & 88 & 81 & 63 & 96 & 54 & 68 & 64 & 54 & 69 & 60 & 61 & 52 & \\
\hline $\mathrm{Sr}$ & 1646 & 1316 & 87 & 1038 & 1416 & 1248 & 822 & 1111 & 1078 & 1215 & 663 & 634 & 694 & 640 & 605 & 676 & \\
\hline Y & 22 & 20 & 5 & 10 & 18 & 21 & 7 & 15 & 17 & 19 & 13 & 10 & 5 & 12 & 8 & 8 & \\
\hline $\mathrm{Zr}$ & 57 & 122 & 109 & 82 & 102 & 107 & 90 & 82 & 96 & 101 & 89 & 102 & 90 & 78 & 92 & 90 & \\
\hline $\mathrm{Nb}$ & 28 & 16 & 15 & 14 & 21 & 21 & 14 & 20 & 12 & 22 & 11 & 13 & 12 & 12 & 14 & 13 & \\
\hline $\mathrm{Ba}$ & 2838 & 2108 & 520 & 1636 & 2656 & 1065 & 1343 & 1614 & 1163 & 1606 & 1341 & 1041 & 1123 & 1712 & 1327 & 1104 & \\
\hline $\mathrm{La}$ & 45 & 49 & ND & 25 & 22 & 18 & 14 & 33 & 32 & 33 & 29 & 12 & 11 & 5 & 22 & 8 & \\
\hline $\mathrm{Ce}$ & 60 & 70 & 22 & 28 & 42 & 61 & 38 & 58 & 56 & 58 & 32 & 35 & 35 & 23 & 22 & 16 & \\
\hline $\mathrm{Nd}$ & 20 & 33 & 7 & 15 & 36 & 17 & 23 & 41 & 18 & 40 & 12 & 13 & 3 & 3 & 15 & ND & \\
\hline Unit & $q m$ & $q m$ & $q m$ & $q m$ & $q m$ & $\mathrm{qm}$ & $q m$ & qm & $\mathrm{qm}$ & $q m$ & $q m$ & qm & qm & $\mathrm{qm}$ & $q m$ & $q m$ & $q m$ \\
\hline Sample & 202855 & 202861 & 202870 & 202871 & 202872 & 202878 & 202884 & 202885 & 202886 & 202926 & 202927 & 202929 & 202930 & 202931 & 202937 & 202938 & 202940 \\
\hline $\mathrm{SiO}_{2}$ & 68.10 & 66.40 & 67.30 & 69.10 & 68.00 & 65.20 & 65.70 & 67.70 & 67.10 & 67.20 & 65.40 & 67.00 & 68.60 & 65.90 & 65.10 & 63.00 & 59.70 \\
\hline $\mathrm{Al}_{2} \mathrm{O}_{3}$ & 16.60 & 16.70 & 16.90 & 16.20 & 16.70 & 17.70 & 17.50 & 16.90 & 16.80 & 16.40 & 16.70 & 17.00 & 15.60 & 17.20 & 17.80 & 17.20 & 17.60 \\
\hline $\mathrm{Fe}_{2} \mathrm{O}_{3}$ & 0.40 & 0.51 & 0.47 & 0.36 & 0.43 & 0.54 & 0.57 & 0.41 & 0.45 & 0.59 & 0.77 & 0.50 & 0.54 & 0.57 & 0.60 & 0.87 & 1.13 \\
\hline $\mathrm{FeO}$ & 1.43 & 1.84 & 1.70 & 1.30 & 1.54 & 1.96 & 2.04 & 1.48 & 1.61 & 2.11 & 2.77 & 1.80 & 1.95 & 2.07 & 2.15 & 3.14 & 4.06 \\
\hline $\mathrm{MgO}$ & 0.42 & 0.43 & 0.49 & 0.42 & 0.54 & 0.70 & 0.65 & 0.45 & 0.54 & 0.62 & 0.79 & 0.52 & 0.66 & 0.65 & 0.61 & 1.05 & 1.48 \\
\hline $\mathrm{CaO}$ & 2.03 & 2.99 & 2.32 & 2.23 & 2.77 & 3.23 & 3.94 & 2.80 & 2.71 & 3.12 & 3.71 & 2.51 & 3.23 & 2.98 & 3.22 & 4.59 & 5.98 \\
\hline $\mathrm{Na}_{2} \mathrm{O}$ & 5.53 & 5.02 & 5.78 & 5.08 & 5.17 & 5.62 & 4.91 & 5.00 & 5.22 & 5.30 & 5.26 & 5.37 & 4.15 & 5.30 & 5.52 & 4.74 & 4.70 \\
\hline $\mathrm{K}_{2} \mathrm{O}$ & 3.85 & 3.94 & 3.39 & 3.24 & 3.09 & 2.98 & 2.70 & 3.39 & 3.57 & 2.88 & 2.91 & 3.72 & 3.47 & 3.56 & 3.56 & 3.19 & 2.88 \\
\hline $\mathrm{TiO}_{2}$ & 0.24 & 0.29 & 0.27 & 0.20 & 0.25 & 0.32 & 0.32 & 0.25 & 0.27 & 0.32 & 0.42 & 0.29 & 0.30 & 0.32 & 0.33 & 0.48 & 0.65 \\
\hline $\mathrm{P}_{2} \mathrm{O}_{5}$ & 0.08 & 0.10 & 0.09 & 0.08 & 0.10 & 0.11 & 0.12 & 0.09 & 0.09 & 0.16 & 0.16 & 0.12 & 0.14 & 0.15 & 0.15 & 0.24 & 0.34 \\
\hline $\mathrm{MnO}$ & 0.07 & 0.09 & 0.07 & 0.04 & 0.07 & 0.09 & 0.10 & 0.10 & 0.07 & 0.11 & 0.10 & 0.08 & 0.09 & 0.09 & 0.08 & 0.12 & 0.15 \\
\hline LOI & 0.21 & 0.66 & 0.13 & 0.40 & 0.25 & 0.22 & 0.40 & 0.30 & 0.25 & 0.26 & 0.18 & 0.01 & ND & 0.09 & ND & 0.21 & 0.14 \\
\hline TOTAL & 98.95 & 98.97 & 98.91 & 98.65 & 98.91 & 98.67 & 98.94 & 98.87 & 98.67 & 99.07 & 99.17 & 98.92 & 98.73 & 98.88 & 99.11 & 98.83 & 98.81 \\
\hline $\mathrm{Rb}$ & 119 & 107 & 65 & 60 & 91 & 79 & 63 & 101 & 112 & 83 & 71 & 72 & 83 & 95 & 129 & 82 & 78 \\
\hline $\mathrm{Sr}$ & 955 & 964 & 1071 & 798 & 865 & 1066 & 1063 & 827 & 925 & 770 & 993 & 987 & 777 & 941 & 878 & 937 & 998 \\
\hline Y & 12 & 16 & 11 & 5 & 16 & 14 & 27 & 17 & 14 & 23 & 24 & 14 & 23 & 16 & 10 & 26 & 28 \\
\hline $\mathrm{Zr}$ & 79 & 134 & 105 & 88 & 90 & 113 & 106 & 85 & 104 & 121 & 135 & 94 & 140 & 106 & 112 & 122 & 105 \\
\hline $\mathrm{Nb}$ & 21 & 19 & 16 & 18 & 15 & 17 & 14 & 18 & 19 & 18 & 16 & 18 & 19 & 21 & 23 & 21 & 10 \\
\hline $\mathrm{Ba}$ & 1701 & 2167 & 1676 & 1299 & 1242 & 1626 & 1396 & 1241 & 1549 & 1050 & 1040 & 1598 & 1373 & 1689 & 1035 & 1395 & 1284 \\
\hline $\mathrm{La}$ & 26 & 39 & 29 & 15 & 15 & 27 & 36 & 27 & 23 & 33 & 31 & 29 & 29 & 33 & 37 & 41 & 40 \\
\hline $\mathrm{Ce}$ & 39 & 65 & 31 & 35 & 37 & 44 & 49 & 56 & 41 & 49 & 82 & 35 & 54 & 53 & 81 & 68 & 48 \\
\hline $\mathrm{Nd}$ & 12 & 29 & 26 & 13 & 12 & 17 & 18 & 13 & 18 & 6 & 37 & 7 & 21 & 21 & 23 & 22 & 16 \\
\hline
\end{tabular}




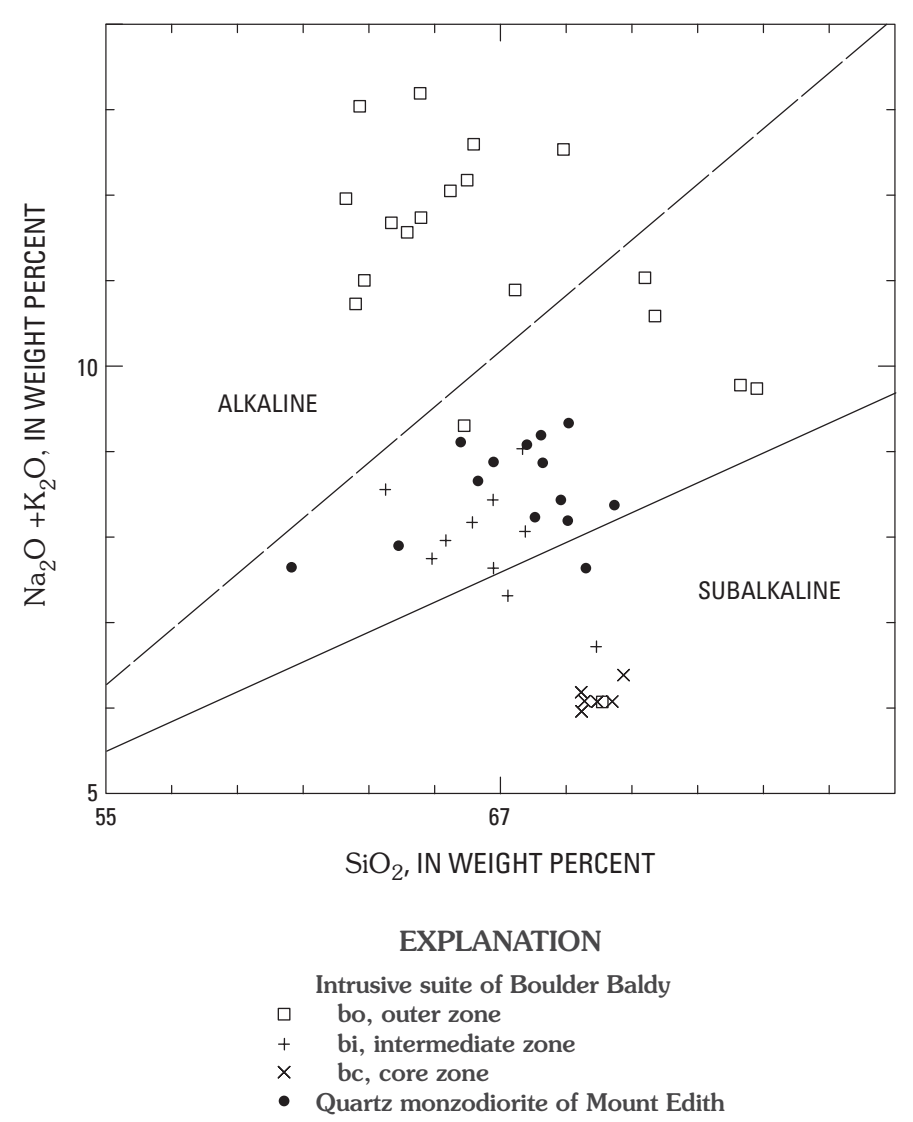

Figure 6. Total alkali-silica variation diagram for intrusions of central Big Belt Mountains. Alkaline-subalkaline dividing lines are those of Irvine and Baragar (1971) (dashes) and Le Bas and others (1986) (solid line).

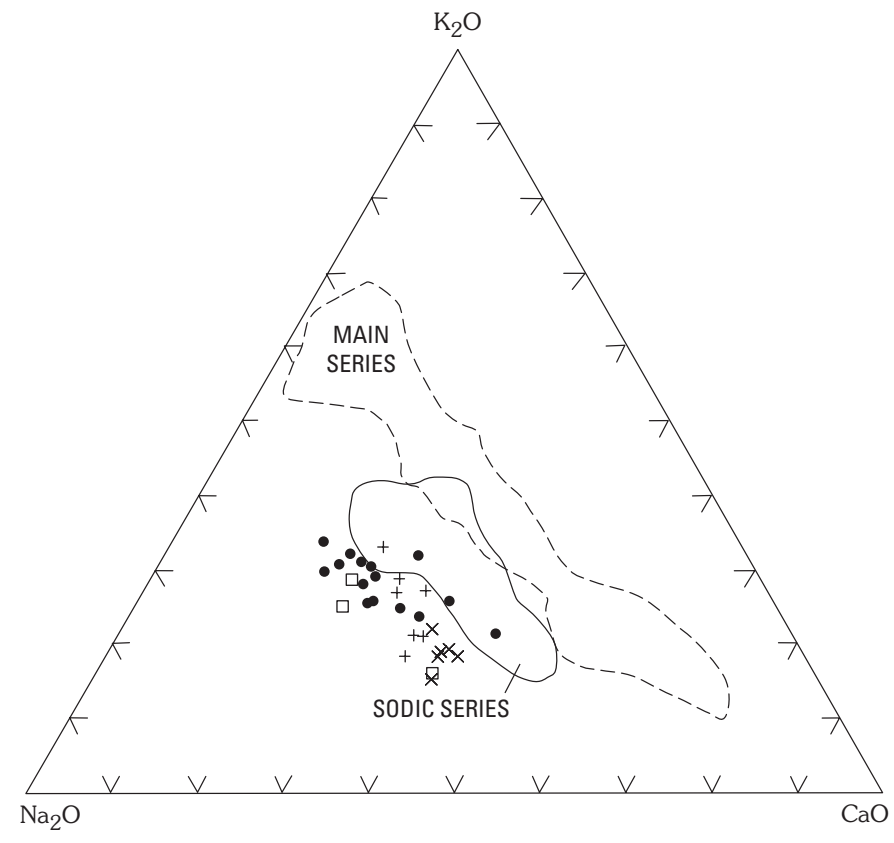

EXPLANATION

Intrusive suite of Boulder Baldy

$\square$ bo, outer zone

$+\quad b i$, intermediate zone

$\times \quad$ bc, core zone

- Quartz monzodiorite of Mount Edith

Figure 7. Variation diagram showing relative proportions of $\mathrm{K}_{2} \mathrm{O}$, $\mathrm{Na}_{2} \mathrm{O}$, and $\mathrm{CaO}$ in intrusions of central Big Belt Mountains. Sodic and main series fields defined by Tilling (1973) for samples of Boulder batholith.

Table 4. Instrumental neutron activation analysis of selected samples of Cretaceous intrusive rocks from central Big Belt Mountains.

[bo, bi, bc, outer, intermediate, and core zones of intrusive suite of Boulder Baldy, respectively; qm, quartz monzodiorite of Mount Edith. All data in parts per million; ND, not detected]

\begin{tabular}{|c|c|c|c|c|c|c|c|c|c|c|c|c|c|c|}
\hline $\begin{array}{l}\text { Unit } \\
\text { Sample }\end{array}$ & $\begin{array}{r}\text { bo } \\
202864\end{array}$ & $\begin{array}{r}\text { bo } \\
202876\end{array}$ & $\begin{array}{r}\text { bo } \\
202882\end{array}$ & $\begin{array}{r}\text { bo } \\
202932\end{array}$ & $\begin{array}{r}\text { bo } \\
202945\end{array}$ & $\begin{array}{r}\text { bi } \\
202934\end{array}$ & $\begin{array}{r}\text { bi } \\
202947\end{array}$ & $\begin{array}{r}b c \\
202854\end{array}$ & $\begin{array}{r}b c \\
202946\end{array}$ & $\begin{array}{r}b c \\
202954\end{array}$ & $\begin{array}{r}q m \\
202870\end{array}$ & $\begin{array}{r}q m \\
202871\end{array}$ & $\begin{array}{r}q m \\
202937\end{array}$ & $\begin{array}{r}q m \\
202940\end{array}$ \\
\hline $\mathrm{Co}$ & 0.330 & 5.54 & 0.170 & 1.43 & 3.10 & 5.24 & 2.34 & 2.50 & 2.08 & 3.95 & 2.52 & 1.78 & 3.00 & 6.03 \\
\hline $\mathrm{Cr}$ & ND & 6.8 & 1.4 & 1.2 & 5.8 & 3.3 & 1.8 & 2.6 & 2.4 & 2.8 & 3.6 & 3.8 & 2.8 & 1.9 \\
\hline $\mathrm{Ni}$ & ND & ND & ND & ND & ND & 11.2 & ND & ND & ND & ND & 7.60 & ND & ND & ND \\
\hline Cs & 0.748 & 0.97 & 1.04 & 4.06 & 1.25 & 1.10 & 2.82 & 0.978 & 1.37 & 1.0 & 0.60 & 0.44 & 1.39 & 0.71 \\
\hline Hf & 8.65 & 4.52 & 3.31 & 2.19 & 6.21 & 3.35 & 3.10 & 2.97 & 2.94 & 3.45 & 3.21 & 2.93 & 4.12 & 3.69 \\
\hline $\mathrm{Sb}$ & 0.053 & ND & 0.046 & 0.077 & 0.061 & ND & 0.043 & 0.030 & ND & ND & 0.055 & ND & ND & 0.063 \\
\hline $\mathrm{Ta}$ & 0.648 & 1.01 & 0.463 & 0.815 & 0.834 & 1.13 & 0.757 & 0.615 & 0.688 & 0.994 & 1.06 & 0.728 & 1.19 & 0.775 \\
\hline Th & 125 & 7.47 & 20.8 & 12.3 & 25.5 & 5.70 & 4.41 & 2.42 & 2.74 & 7.59 & 4.38 & 5.11 & 8.34 & 5.62 \\
\hline $\mathrm{U}$ & 4.8 & 2.3 & 2.9 & 4.6 & 3.9 & 1.5 & 1.2 & 0.78 & 1.1 & 1.6 & 1.0 & 1.2 & 1.9 & 1.4 \\
\hline $\mathrm{Zn}$ & 78.0 & 58.1 & 63.9 & 51.9 & 54.9 & 46 & 43.5 & 59.1 & 51.2 & 41 & 35 & 21 & 23 & 45 \\
\hline $\mathrm{Sc}$ & 0.143 & 4.06 & 0.406 & 0.40 & 3.07 & 4.18 & 1.98 & 1.78 & 1.67 & 3.30 & 2.36 & 1.72 & 2.79 & 7.87 \\
\hline $\mathrm{La}$ & 2.5 & 41.8 & 22.5 & 71.2 & 40.0 & 28.4 & 21.9 & 14.8 & 15.6 & 26.2 & 21.3 & 14.9 & 37.6 & 35.8 \\
\hline $\mathrm{Ce}$ & 7.3 & 78.1 & 36.1 & 91.2 & 63.5 & 52.1 & 34.6 & 25.9 & 27 & 45.4 & 38 & 25.0 & 57.3 & 64.3 \\
\hline $\mathrm{Nd}$ & ND & 31 & 10 & 21 & 24 & 22 & 11 & 10 & 11 & 19 & 15 & 10 & 19 & 30 \\
\hline $\mathrm{Sm}$ & 0.51 & 6.36 & 0.92 & 2.5 & 4.41 & 4.58 & 2.14 & 2.05 & 2.34 & 3.54 & 2.95 & 1.89 & 3.71 & 6.73 \\
\hline $\mathrm{Eu}$ & 0.11 & 1.50 & 0.15 & 0.548 & 1.16 & 1.24 & 0.561 & 0.635 & 0.646 & 0.91 & 0.772 & 0.541 & 0.976 & 1.77 \\
\hline $\mathrm{Tb}$ & 0.073 & 0.614 & 0.059 & 0.14 & 0.457 & 0.482 & 0.23 & 0.214 & 0.24 & 0.372 & 0.33 & 0.21 & 0.414 & 0.789 \\
\hline $\mathrm{Yb}$ & 0.67 & 2.3 & 0.29 & 0.46 & 2.1 & 1.9 & 0.99 & 0.75 & 0.80 & 1.5 & 1.3 & 1.1 & 1.9 & 2.5 \\
\hline $\mathrm{Lu}$ & 0.11 & 0.29 & 0.039 & 0.053 & 0.306 & 0.26 & 0.14 & 0.099 & 0.10 & 0.21 & 0.18 & 0.15 & 0.28 & 0.34 \\
\hline
\end{tabular}


Ta, Hf, Cs, Rb, and Y display no consistent covariation with $\mathrm{SiO}_{2}$ content (table 3). With the exception of three of five samples of the quartz monzonite of the intrusive suite of Boulder Baldy, chondrite-normalized extended trace-element patterns for intrusive rocks of the central Big Belt Mountains are gently negatively sloping and are characterized by variably well developed negative $\mathrm{Nb}-\mathrm{Ta}, \mathrm{P}$, and $\mathrm{Ti}$ anomalies and positive $\mathrm{Ba}, \mathrm{K}$, and $\mathrm{Sr}$ anomalies (fig. 8). In most samples, abundances of $\mathrm{Rb}$ and $\mathrm{Yb}$ (at opposite ends of chondrite-normalized extended trace-element patterns) are between about 155-250 and 3-10 times chondritic values, respectively. Patterns for samples of the central Big Belt Mountains intrusions are essentially parallel and define relatively limited compositional variation. Trace-element contents of the biotite granodiorite are systematically lower than those of hornblende quartz monzodiorite. Extended trace-element patterns for the hornblende quartz monzodiorites of Mount Edith and the intrusive suite of Boulder Baldy are indistinguishable. The slopes and shapes of these patterns, as well as trace-element abundances, are similar to those of back-arc, subduction-related rocks, including, for example, well-characterized middle Tertiary ash-flow tuffs of the southern Great Basin (du Bray, 1995b).

Extended trace-element patterns for the two quartz monzonite samples that best preserve igneous textures have slopes and shapes similar to those of the majority of central Big Belt Mountains intrusions but indicate slightly higher traceelement abundances. The three remaining samples of the quartz monzonite have extended trace-element patterns that are somewhat more steeply negatively sloping (fig. $8 B$ ) than those of other central Big Belt Mountains intrusions. In particular, two of these patterns are distinguished by large negative $\mathrm{Ba}$ anomalies and large positive $\mathrm{Zr}$ anomalies. The central parts of these three patterns are considerably irregular and depict broad traceelement abundance ranges.

With the exception of the three unusual samples of the quartz monzonite of the intrusive suite of Boulder Baldy, chondrite-normalized REE patterns for the central Big Belt Mountains intrusions are gently negatively sloping in their light REE parts, lack Eu anomalies, and are essentially flat in their heavy REE parts (fig. 9A). The patterns depict a narrow compositional range and are remarkably parallel. $(\mathrm{La} / \mathrm{Yb})_{\mathrm{CN}}$ values vary from about 11 to 16 . REE contents are systematically lower in samples with higher $\mathrm{SiO}_{2}$ and lower $\mathrm{TiO}_{2}$ and $\mathrm{MgO}$ contents. Accordingly, the REE contents of the biotite granodiorite are systematically lower than those of hornblende quartz monzodiorite. REE patterns for the hornblende quartz monzodiorites of Mount Edith and of the intrusive suite of Boulder Baldy are indistinguishable.

Rare-earth element patterns for the two quartz monzonite samples that best preserve igneous textures have slopes similar to those of the majority of central Big Belt Mountains intrusions but indicate slightly higher REE abundances. The three remaining samples of the quartz monzonite have REE patterns that are unusual (fig. 9B) relative to those for other central Big Belt Mountains intrusions. One of the three unusual patterns indicates distinctly low REE abundances and is essentially flat with minor middle REE depletion. The other two unusual patterns are distinctly more negatively sloping in their light REE parts; the heavy REE parts of these patterns are flat and indicate abundances significantly lower than is characteristic of other central Big Belt Mountains intrusions. The distinctiveness of these three patterns, along with their petrographic features, suggests that these rocks were acted upon by processes that did not affect the majority of the central Big Belt Mountains intrusions.

\section{Mineral Compositions}

Compositions of subhedral to euhedral, non-interstitial minerals that may have participated in chemical evolution of the central Big Belt Mountains igneous rocks, including plagioclase, biotite, hornblende, potassium feldspar, clinopyroxene, titanite, apatite, magnetite, and andradite, were determined using a JEOL 8900 electron microprobe with five wavelength dispersive crystal spectrometers. A 5-20 $\mu \mathrm{m}$ beam, with $15 \mathrm{kV}$ accelerating voltage and $20 \mathrm{nA}$ beam current, was used to make a minimum of three 20-s analyses on three grains of each selected mineral in the representative sample subset. Most analyzed spots were at crystal rims to ensure that determined compositions reflect the composition of the interstitial, residual liquid from which the analyzed minerals crystallized.

The outer rims of plagioclase crystals (table 5; fig. 10) in samples of the central Big Belt Mountains igneous rocks have average compositions that range from andesine $\left(\mathrm{An}_{35} \mathrm{Ab}_{64} \mathrm{Or}_{1}\right)$ to albite $\left(\mathrm{An}_{3} \mathrm{Ab}_{96} \mathrm{Or}_{1}\right)$; most are composed of calcic oligoclase. Compositions of plagioclase in the two quartz monzodiorite intrusions are similar. Plagioclase in the granodiorite is distinctly more calcic, whereas sparse plagioclase in the quartz monzonite is distinctly less calcic than plagioclase in the quartz monzodiorite. Potassium feldspar (table 6; fig. 10) is orthoclase and has compositions that range from $\mathrm{An}_{0} \mathrm{Ab}_{22} \mathrm{Or}_{76} \mathrm{Cs}_{2}$ to $\mathrm{An}_{0} \mathrm{Ab}_{95} \mathrm{Or}_{5}$. Compositions of potassium feldspar in the two quartz monzodiorites are indistinguishable. Potassium feldspar in the granodiorite is distinctly more potassic, whereas that in two of three samples of the quartz monzonite is distinctly more sodic than that in the quartz monzodiorite. Reconnaissance analyses across single feldspar crystals indicate that core-to-rim zoning is limited to about $5 \mathrm{~mol}$ percent in most grains; however, feldspar grains in a few analyzed samples display as much as $15 \mathrm{~mol}$ percent compositional variation. Potassium feldspar in several analyzed samples contains considerable $\mathrm{BaO}$; orthoclase 202932, in particular, contains an average of 1.09 weight percent $\mathrm{BaO}$.

The composition of biotite in the central Big Belt Mountains igneous rocks (table 7; fig. 11) is similar to that of magnesium-rich biotite in granitoids of the Sierra Nevada batholith (Dodge and others, 1969). Biotite in the central Big Belt Mountains igneous rocks has slightly elevated $\mathrm{BaO}$ contents $(0.45-$ 0.87 weight percent), relative to Sierra Nevada biotite;

$\mathrm{MgO}: \mathrm{FeO}^{*}$ is about 1 , and they contain slightly less magnesium and distinctly less titanium than biotite in the Big Timber stock (du Bray and Harlan, 1996), in the nearby Crazy Mountains. Compositional variation of biotite within individual samples is quite limited; no zonation was detected.

Amphibole is the principal mafic silicate mineral in the quartz monzodiorite intrusions of the central Big Belt Mountains. Iron contents of analyzed hornblendes were partitioned to 

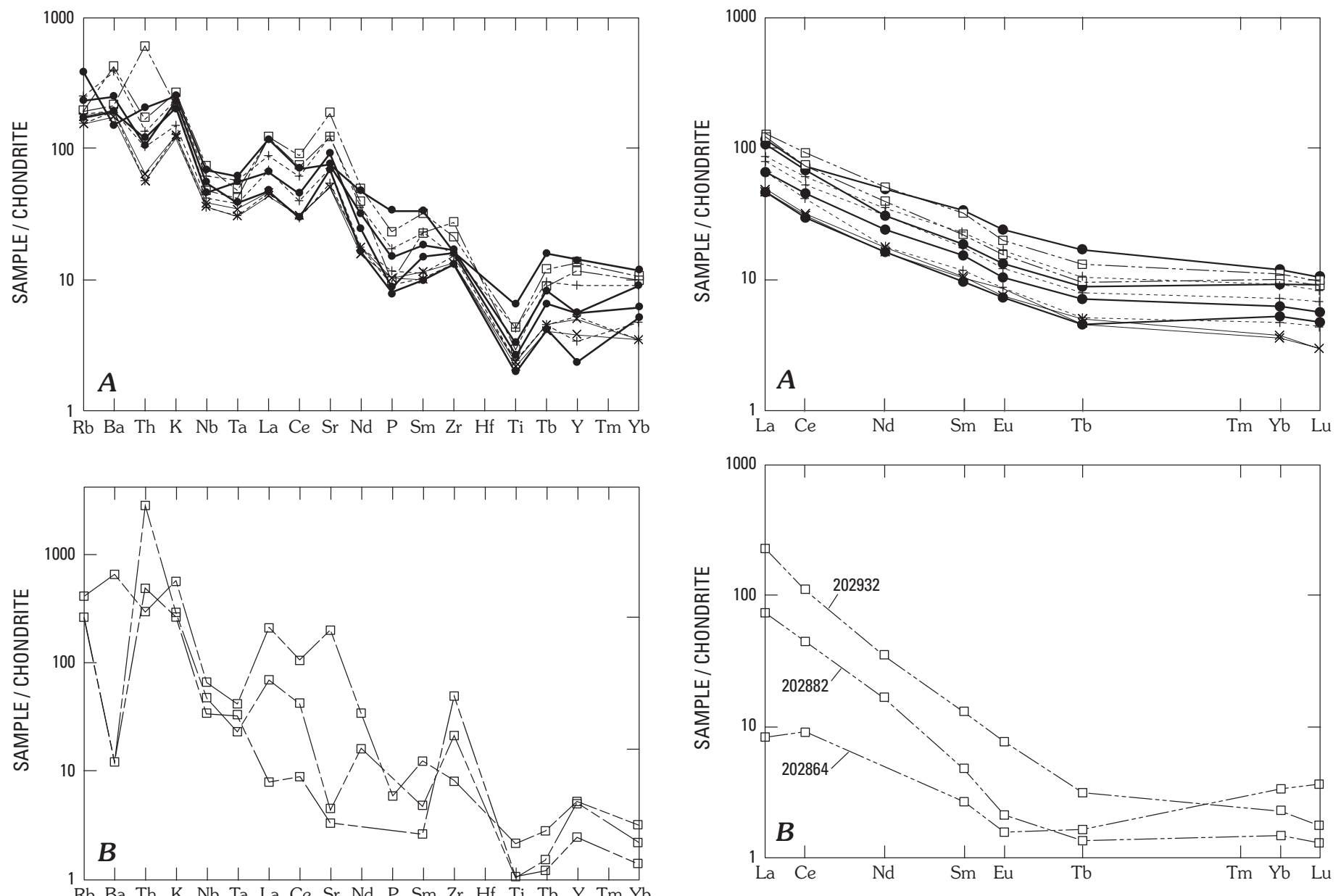

\section{EXPLANATION}

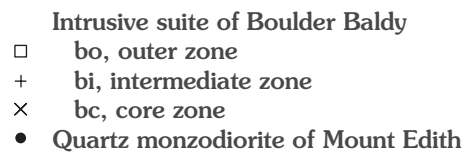

Figure 8. Chondrite-normalized extended trace-element diagrams showing compositions of central Big Belt Mountains intrusions. A, Patterns for all analyzed samples except those for three petrographically and geochemically unusual samples of the quartz monzonite of the intrusive suite of Boulder Baldy. B, Patterns for three petrographically and geochemically unusual samples of the quartz monzonite of the intrusive suite of Boulder Baldy. All points in $B$ are (bo).

\section{EXPLANATION}

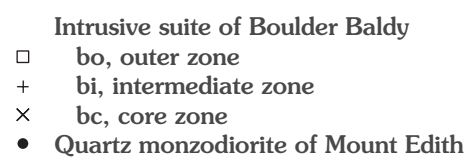

Figure 9. Chondrite-normalized rare-earth element diagrams showing compositions of central Big Belt Mountains intrusions. A, Patterns for all analyzed samples except those for three petrographically and geochemically unusual samples of the quartz monzonite of the intrusive suite of Boulder Baldy. $B$, Patterns for three petrographically and geochemically unusual samples of the quartz monzonite of the intrusive suite of Boulder Baldy. All points in $B$ are (bo).

Table 5. Major oxide compositions of plagioclase in intrusive rocks from central Big Belt Mountains.

[bo, bi, bc, outer, intermediate, and core zones of intrusive suite of Boulder Baldy, respectively; qm, quartz monzodiorite of Mount Edith. Data in weight percent; each analysis is the average of nine analyses (three spots on each of three grains)]

\begin{tabular}{|c|c|c|c|c|c|c|c|c|c|}
\hline $\begin{array}{l}\text { Unit } \\
\text { Sample }\end{array}$ & $\begin{array}{r}\text { bo } \\
876 \mathrm{PL}\end{array}$ & $\begin{array}{r}\text { bo } \\
\text { 932PL }\end{array}$ & $\begin{array}{r}\text { bi } \\
934 P L\end{array}$ & $\begin{array}{r}\text { bi } \\
947 P L\end{array}$ & $\begin{array}{r}\text { bc } \\
854 P L\end{array}$ & $\begin{array}{r}\text { bc } \\
946 \mathrm{PL}\end{array}$ & $\begin{array}{l}\text { qm } \\
871 P L\end{array}$ & $\begin{array}{r}q m \\
937 P L\end{array}$ & $\begin{array}{r}\text { qm } \\
940 P L\end{array}$ \\
\hline $\mathrm{SiO}_{2}$ & 66.67 & 67.83 & 62.36 & 62.01 & 59.89 & 59.38 & 63.81 & 62.74 & 61.14 \\
\hline $\mathrm{Al}_{2} \mathrm{O}_{3}$ & 20.82 & 19.86 & 23.17 & 23.79 & 25.53 & 25.69 & 22.35 & 23.54 & 25.05 \\
\hline $\mathrm{FeO}$ & 0.13 & 0.10 & 0.14 & 0.13 & 0.12 & 0.15 & 0.14 & 0.15 & 0.14 \\
\hline $\mathrm{BaO}$ & 0.03 & 0.02 & 0.02 & 0.03 & 0.02 & 0.02 & 0.03 & 0.02 & 0.03 \\
\hline $\mathrm{CaO}$ & 1.56 & 0.58 & 4.78 & 5.38 & 6.79 & 7.45 & 3.62 & 5.17 & 6.44 \\
\hline $\mathrm{Na}_{2} \mathrm{O}$ & 10.77 & 11.22 & 8.53 & 8.50 & 7.84 & 7.37 & 9.47 & 8.73 & 8.12 \\
\hline $\mathrm{K}_{2} \mathrm{O}$ & 0.12 & 0.13 & 0.32 & 0.36 & 0.19 & 0.16 & 0.26 & 0.25 & 0.20 \\
\hline Total & 100.11 & 99.74 & 99.32 & 100.20 & 100.38 & 100.21 & 99.67 & 100.61 & 101.13 \\
\hline
\end{tabular}


Table 6. Compositions of potassium feldspar in intrusive rocks from central Big Belt Mountains.

\begin{tabular}{|c|c|c|c|c|c|c|c|c|}
\hline $\begin{array}{l}\text { Unit } \\
\text { Sample }\end{array}$ & $\begin{array}{r}\text { bo } \\
876 \mathrm{KF}\end{array}$ & $\begin{array}{r}\text { bo } \\
882 \mathrm{KF}\end{array}$ & $\begin{array}{r}\text { bo } \\
932 \mathrm{KF}\end{array}$ & $\begin{array}{r}\text { bi } \\
934 K F\end{array}$ & $\begin{array}{r}b i \\
947 \mathrm{KF}\end{array}$ & $\begin{array}{r}b c \\
854 K F\end{array}$ & $\begin{array}{r}q m \\
871 \mathrm{KF}\end{array}$ & $\begin{array}{r}q m \\
937 \mathrm{KF}\end{array}$ \\
\hline $\mathrm{SiO}_{2}$ & 63.83 & 64.93 & 64.32 & 65.17 & 63.92 & 65.73 & 63.42 & 64.91 \\
\hline $\mathrm{Al}_{2} \mathrm{O}_{3}$ & 18.98 & 17.94 & 18.14 & 18.03 & 18.78 & 18.63 & 18.69 & 18.75 \\
\hline $\mathrm{FeO}$ & 0.09 & 0.36 & 0.18 & 0.07 & 0.05 & 0.05 & 0.09 & 0.09 \\
\hline $\mathrm{BaO}$ & 1.49 & 0.01 & 1.09 & 0.46 & 1.82 & 0.12 & 1.12 & 0.56 \\
\hline $\mathrm{CaO}$ & 0.13 & 0.00 & 0.02 & 0.06 & 0.03 & 0.01 & 0.03 & 0.04 \\
\hline $\mathrm{Na}_{2} \mathrm{O}$ & 2.33 & 0.60 & 2.38 & 1.43 & 1.32 & 0.98 & 1.42 & 1.53 \\
\hline $\mathrm{K}_{2} \mathrm{O}$ & 12.76 & 15.79 & 12.64 & 14.09 & 14.06 & 15.37 & 14.28 & 14.09 \\
\hline Total & 99.61 & 99.64 & 98.77 & 99.32 & 99.98 & 100.89 & 99.04 & 99.98 \\
\hline
\end{tabular}

Table 7. Compositions of biotite in intrusive rocks from central Big Belt Mountains.

[bi, bc, intermediate and core zones of intrusive suite of Boulder Baldy, respectively. Data in weight percent; each analysis is the average of nine analyses (three spots on each of three grains); iron contents partitioned assuming $\mathrm{Fe}^{2+} /\left(\mathrm{Fe}^{2+}+\mathrm{Fe}^{3+}\right)=0.85 ; \mathrm{H}_{2} \mathrm{O}$ calculated by stoichiometry; Total $_{\mathrm{a}}$, total adjusted for $\mathrm{F}$ and $\mathrm{Cl}]$

\begin{tabular}{lrrr}
\hline Unit & bi & $b c$ & $b c$ \\
Sample & $947 \mathrm{BI}$ & $854 \mathrm{BI}$ & $946 \mathrm{BI}$ \\
\hline $\mathrm{SiO}_{2}$ & 36.87 & 36.87 & 36.17 \\
$\mathrm{TiO}_{2}$ & 2.39 & 3.26 & 3.13 \\
$\mathrm{Al}_{2} \mathrm{O}_{3}$ & 14.51 & 15.00 & 14.75 \\
$\mathrm{Fe}_{2} \mathrm{O}_{3}$ & 3.10 & 3.17 & 3.24 \\
$\mathrm{FeO}$ & 15.80 & 16.18 & 16.53 \\
$\mathrm{MnO}$ & 1.40 & 1.11 & 1.12 \\
$\mathrm{MgO}$ & 11.19 & 10.55 & 10.10 \\
$\mathrm{BaO}$ & 0.45 & 0.87 & 0.77 \\
$\mathrm{Na} a_{2} \mathrm{O}$ & 0.08 & 0.09 & 0.10 \\
$\mathrm{~K}_{2} \mathrm{O}$ & 9.35 & 9.40 & 9.36 \\
$\mathrm{~F}$ & 0.51 & 0.54 & 0.63 \\
$\mathrm{Cl}$ & 0.01 & 0.01 & 0.01 \\
$\mathrm{H}_{2} \mathrm{O}$ & 3.67 & 3.70 & 3.60 \\
$\mathrm{Total}_{\text {Total }}$ & 99.34 & 100.74 & 99.51 \\
\hline
\end{tabular}

ferrous and ferric iron by charge balancing; $\mathrm{H}_{2} \mathrm{O}$ contents were calculated by stoichiometry (table 8 ). According to the classification scheme of Leake (1978), the amphibole (table 8; fig. 12), with $(\mathrm{Ca}+\mathrm{Na})_{\mathrm{B}}>1.34, \mathrm{Na}_{\mathrm{B}}<0.5,0.5>\mathrm{Mg} /\left(\mathrm{Mg}+\mathrm{Fe}^{2+}\right)>1.0$, and $7.25<\mathrm{Si}<6.5$, is magnesio-hornblende. Most of the magnesiohornblende displays limited within-sample compositional variation, and zoning was not detected. As a group, the magnesiohornblendes display a moderate amount of between-sample compositional variation with respect to iron and magnesium contents. Hornblende from quartz monzodiorite of the intrusive suite of Boulder Baldy has a somewhat higher iron-magnesium ratio than that in the quartz monzodiorite of Mount Edith (fig.

12). Hornblende in intrusions of the central Big Belt Mountains also has a slightly higher iron/magnesium ratio than is characteristic of hornblende in the nearby Big Timber stock (du Bray and Harlan, 1996).

The most mafic parts of quartz monzodiorite intrusions in the central Big Belt Mountains contain small amounts of
Table 8. Compositions of hornblende in intrusive rocks from central Big Belt Mountains.

[bi, qm, intermediate zone of intrusive suite of Boulder Baldy and quartz monzodiorite of Mount Edith, respectively. Data in weight percent; each analysis is the average of nine analyses (three spots on each of three grains); $\mathrm{Fe}_{2} \mathrm{O}_{3}$ and $\mathrm{H}_{2} \mathrm{O}$ calculated by charge balance and stoichiometry, respectively; Total $_{\mathrm{a}}$, total adjusted for F]

\begin{tabular}{lrrrrr}
\hline Unit & bi & bi & qm & qm & qm \\
Sample & $934 \mathrm{HB}$ & $947 \mathrm{HB}$ & $871 \mathrm{HB}$ & $937 \mathrm{HB}$ & $940 \mathrm{HB}$ \\
\hline $\mathrm{SiO}_{2}$ & 44.62 & 44.28 & 48.92 & 48.10 & 47.44 \\
$\mathrm{TiO}_{2}$ & 1.11 & 1.09 & 0.54 & 0.63 & 0.94 \\
$\mathrm{Al}_{2} \mathrm{O}_{3}$ & 8.43 & 9.34 & 5.25 & 6.48 & 7.08 \\
$\mathrm{Fe}_{2} \mathrm{O}_{3}$ & 3.32 & 4.92 & 4.22 & 2.93 & 2.61 \\
$\mathrm{FeO}$ & 14.35 & 12.84 & 10.25 & 13.05 & 13.90 \\
$\mathrm{MnO}$ & 1.07 & 1.42 & 1.22 & 1.18 & 0.94 \\
$\mathrm{MgO}$ & 10.35 & 10.15 & 13.42 & 12.16 & 11.75 \\
$\mathrm{CaO}$ & 11.38 & 11.39 & 11.40 & 11.41 & 11.68 \\
$\mathrm{Na} 2$ & 1.87 & 1.54 & 1.37 & 1.84 & 1.73 \\
$\mathrm{~K}_{2} \mathrm{O}$ & 1.02 & 1.07 & 0.58 & 0.85 & 0.79 \\
$\mathrm{H}_{2} \mathrm{O}$ & 1.80 & 1.86 & 1.79 & 1.59 & 1.72 \\
$\mathrm{~F}$ & 0.37 & 0.26 & 0.49 & 0.92 & 0.65 \\
$\mathrm{Total}$ & 99.68 & 100.16 & 99.45 & 101.15 & 101.24 \\
Total $_{\mathrm{a}}$ & 99.52 & 100.05 & 99.24 & 100.76 & 100.96 \\
\hline
\end{tabular}

clinopyroxene. The clinopyroxene is salite, whose composition is $\mathrm{Wo}_{49} \mathrm{En}_{34} \mathrm{Fs}_{17}$ (table 9; fig. 12); the $\mathrm{MnO}$ content of the salite, 1.2 to 1.5 weight percent, is somewhat distinctive. Within- and between-sample clinopyroxene compositional variation is limited, and zoning was not detected; salite in the two quartz monzodiorite intrusions is compositionally indistinguishable. Clinopyroxene in the quartz monzodiorites is more calcic than that in the nearby Big Timber stock (du Bray and Harlan, 1996).

The composition of pyroxene in the quartz monzonite of the intrusive suite of Boulder Baldy ranges from aegirine-augite to aegirine (table 9). Pyroxene in sample 202882 contains 0.7 atoms of $\mathrm{Fe}^{3+}$ per formula unit and 9.8 weight percent $\mathrm{Na}_{2} \mathrm{O}$; these features and the pleochroism of this pyroxene are diagnostic of aegirine. Pyroxene in samples 202876 and 202932 contains 0.15 and 0.30 atoms of $\mathrm{Fe}^{3+}$ per formula unit and 2.0 and 3.8 weight percent $\mathrm{Na}_{2} \mathrm{O}$, respectively; these features and the pleochroism of these pyroxenes are diagnostic of 


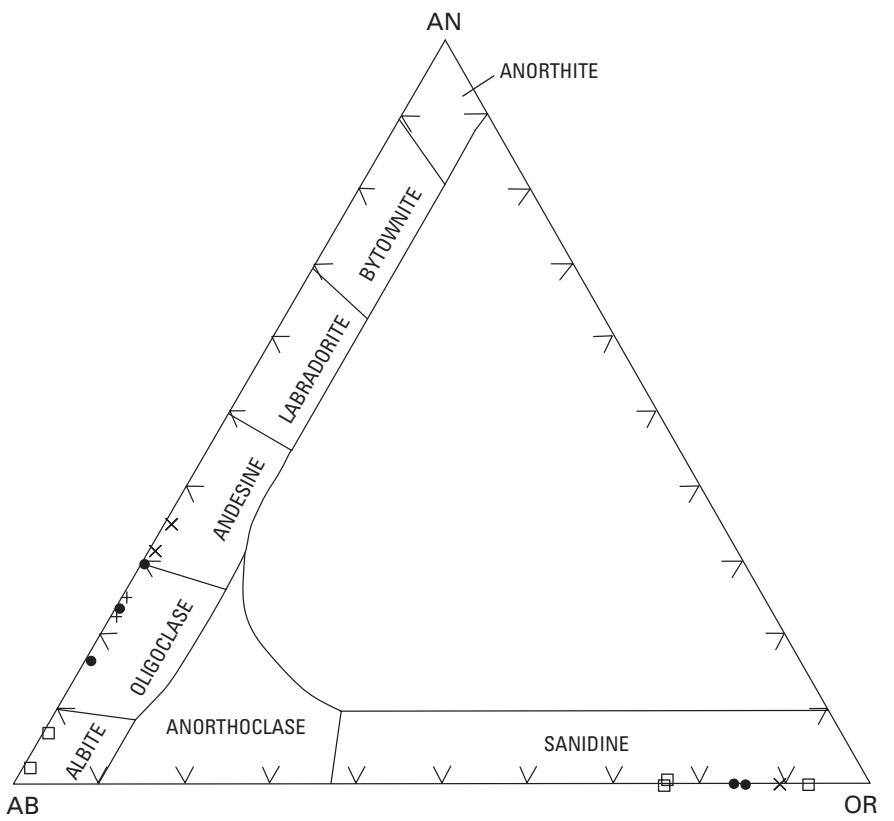

\section{EXPLANATION}

Intrusive suite of Boulder Baldy

$\square$ bo, outer zone

$+\quad$ bi, intermediate zone

$\times$ bc, core zone

- Quartz monzodiorite of Mount Edith

Figure 10. Ternary diagram showing molecular end member proportions of feldspars in samples of central Big Belt Mountains intrusions.

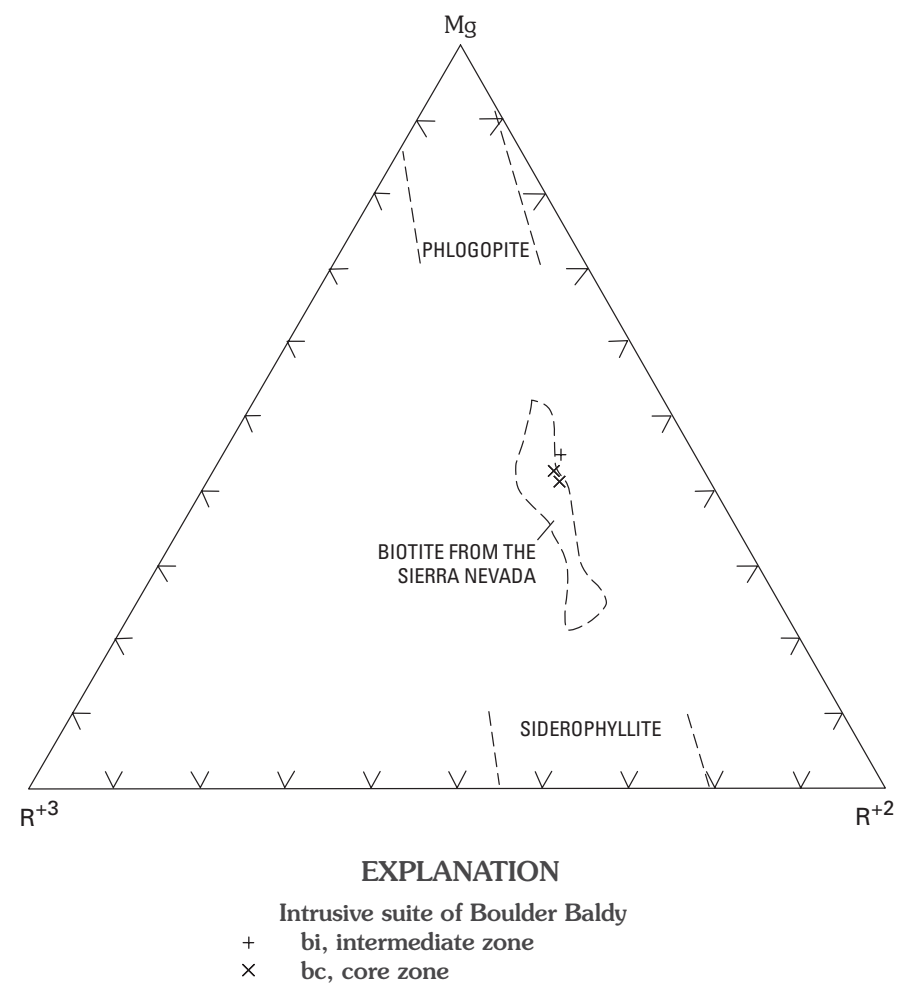

Figure 11. Ternary $\mathrm{Mg}, \mathrm{R}^{+3}\left(\mathrm{Al}^{\mathrm{IV}}+\mathrm{Fe}^{3+}+\mathrm{Ti}\right), \mathrm{R}^{+2}\left(\mathrm{Fe}^{2+}+\mathrm{Mn}\right)$ diagram showing relative cation abundances for biotite in samples of central Big Belt Mountains intrusions.

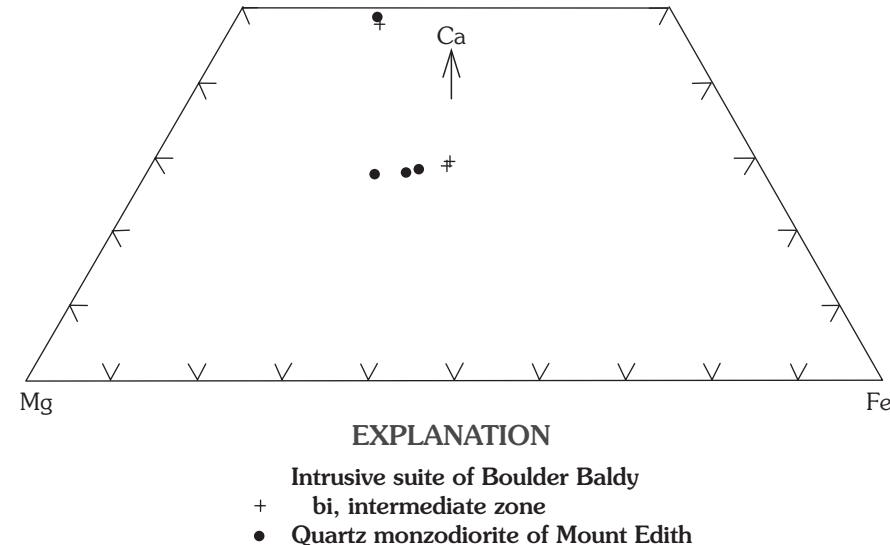

Figure 12. Ternary diagram showing compositions of amphibole and clinopyroxene in quartz monzodiorite of central Big Belt Mountains intrusions. Clinopyroxene compositions are those which plot near the top of the composition quadrilateral.

aegirine-augite. Sodic pyroxenes such as this are common constituents of alkaline igneous rocks, which accords with the compositions of their host rocks.

Representative compositions of accessory minerals in igneous rocks of the central Big Belt Mountains, including titanite, apatite, magnetite, and andradite, are summarized in table 10. Titanite compositions from four samples are similar; deviations from analytical totals of 100 percent probably reflect undetermined components, including $\mathrm{H}_{2} \mathrm{O}, \mathrm{Zr}, \mathrm{Nb}$, Ta, $\mathrm{V}$, and the REE. Similarly, apatite compositions from three samples display remarkably little variation; these apatites contain notably elevated fluorine abundances. Opaque oxide grains in intrusions of the central Big Belt Mountains are composed of magnetite; magnetite compositions in four analyzed samples are indistinguishable. The most unusual mineralogic feature of igneous rocks in the central Big Belt Mountains is the presence of garnet in the outer zone of the intrusive suite of Boulder Baldy. In terms of end-member components, this garnet is primarily andradite ( 87 percent) but includes grossularite (10 percent) and spessartine ( 3 percent) components as well. The elevated $\mathrm{TiO}_{2}$ content (table

Table 9. Compositions of clinopyroxene in intrusive rocks from central Big Belt mountains.

[bo, bi, outer and intermediate zones of intrusive suite of Boulder Baldy, respectively; qm, quartz monzodiorite of Mount Edith. Data in weight percent; each analysis is the average of nine analyses (three spots on each of three grains)]

\begin{tabular}{lrrrrr}
\hline $\begin{array}{l}\text { Unit } \\
\text { Sample }\end{array}$ & $\begin{array}{r}\text { bo } \\
\text { 876CP }\end{array}$ & $\begin{array}{r}\text { bo } \\
882 \mathrm{CP}\end{array}$ & $\begin{array}{r}\text { bo } \\
\text { 932CP }\end{array}$ & $\begin{array}{r}\text { bi } \\
\text { 934CP }\end{array}$ & $\begin{array}{r}\text { qm } \\
\text { 940CP }\end{array}$ \\
\hline $\mathrm{SiO}_{2}$ & 51.85 & 52.74 & 50.27 & 52.68 & 53.14 \\
$\mathrm{TiO}_{2}$ & 0.11 & 0.18 & 0.20 & 0.06 & 0.12 \\
$\mathrm{Al}_{2} \mathrm{O}_{3}$ & 1.13 & 0.34 & 2.20 & 0.71 & 0.79 \\
$\mathrm{FeO}$ & 14.04 & 24.32 & 17.80 & 10.41 & 9.96 \\
$\mathrm{MnO}$ & 0.96 & 2.28 & 1.30 & 1.49 & 1.21 \\
$\mathrm{MgO}$ & 9.00 & 1.79 & 5.78 & 11.26 & 11.64 \\
$\mathrm{CaO}$ & 20.62 & 7.59 & 17.55 & 22.61 & 23.20 \\
$\mathrm{Na}$ & 2.01 & 9.84 & 3.84 & 0.54 & 0.56 \\
$\mathrm{Total}$ & 99.72 & 99.08 & 98.93 & 99.76 & 100.61 \\
\hline
\end{tabular}


Table 10. Representative compositions of accessory phases in intrusive rocks from central Big Belt Mountains.

[bo, bi, bc, outer, intermediate, and core zones of intrusive suite of Boulder Baldy, respectively; qm, quartz monzodiorite of Mount Edith. Data in weight percent; each analysis is the average of nine analyses (three spots on each of three grains); $\mathrm{Fe}_{2} \mathrm{O}_{3}$ in magnetite calculated by charge balance; $\mathrm{ND}$, not detected; Total $_{\mathrm{a}}$, total adjusted for $\mathrm{F}$ and $\left.\mathrm{Cl}\right]$

\begin{tabular}{|c|c|c|c|c|c|c|c|c|c|c|c|c|}
\hline \multirow{3}{*}{$\begin{array}{l}\text { Unit } \\
\text { Sample }\end{array}$} & bo & bi & bc & $q m$ & bi & bc & $q m$ & bo & bi & bc & $q m$ & bo \\
\hline & 932SP & 947SP & $854 \mathrm{SP}$ & 937SP & 947AP & 854AP & 937AP & $9320 \mathrm{P}$ & $9470 P$ & $8540 P$ & $9370 \mathrm{P}$ & 932GR \\
\hline & \multicolumn{4}{|c|}{ Titanite } & \multicolumn{3}{|c|}{ Apatite } & \multicolumn{4}{|c|}{ Magnetite } & Andradite \\
\hline $\mathrm{SiO}_{2}$ & 30.05 & 30.39 & 30.79 & 30.36 & ND & ND & ND & ND & ND & ND & ND & 35.20 \\
\hline $\mathrm{TiO}_{2}$ & 34.60 & 35.69 & 36.14 & 36.23 & ND & ND & ND & 0.07 & 0.07 & 0.13 & 0.21 & 2.48 \\
\hline $\mathrm{Al}_{2} \mathrm{O}_{3}$ & 1.42 & 1.68 & 1.61 & 1.50 & ND & ND & ND & 0.15 & 0.13 & 0.10 & 0.19 & 2.59 \\
\hline $\mathrm{Cr}_{2} \mathrm{O}_{3}$ & ND & ND & ND & ND & ND & ND & ND & 0.01 & 0.02 & 0.02 & 0.03 & ND \\
\hline $\mathrm{Fe}_{2} \mathrm{O}_{3}$ & ND & ND & ND & ND & ND & ND & ND & 67.53 & 68.13 & 67.98 & 67.59 & 26.53 \\
\hline $\mathrm{FeO}$ & 2.16 & 1.43 & 1.27 & 1.42 & 0.26 & 0.27 & 0.02 & 29.66 & 29.86 & 30.53 & 29.85 & 0.32 \\
\hline $\mathrm{MnO}$ & 0.05 & 0.23 & 0.26 & 0.21 & 0.20 & 0.20 & 0.11 & 0.92 & 0.99 & 0.36 & 1.03 & 1.36 \\
\hline $\mathrm{MgO}$ & ND & ND & ND & ND & ND & ND & ND & 0.01 & 0.01 & 0.00 & 0.02 & 0.15 \\
\hline $\mathrm{BaO}$ & 0.25 & 0.24 & 0.25 & 0.27 & ND & ND & ND & ND & ND & ND & ND & ND \\
\hline $\mathrm{CaO}$ & 27.50 & 27.84 & 28.17 & 27.98 & 55.59 & 56.20 & 55.71 & ND & ND & ND & ND & 31.64 \\
\hline $\mathrm{Na}_{2} \mathrm{O}$ & ND & ND & ND & ND & ND & ND & ND & ND & ND & ND & ND & 0.08 \\
\hline $\mathrm{P}_{2} \mathrm{O}_{5}$ & ND & ND & ND & ND & 41.81 & 41.85 & 41.44 & ND & ND & ND & ND & ND \\
\hline $\mathrm{F}$ & 0.41 & 0.29 & 0.32 & 0.30 & 3.25 & 3.76 & 4.42 & ND & ND & ND & ND & ND \\
\hline $\mathrm{Cl}$ & ND & ND & ND & ND & 0.00 & 0.01 & 0.02 & ND & ND & ND & ND & ND \\
\hline Total & 96.44 & 97.79 & 98.81 & 98.27 & 101.11 & 102.29 & 101.72 & 98.35 & 99.21 & 99.12 & 98.92 & 100.26 \\
\hline Total $_{\mathrm{a}}$ & 96.27 & 97.67 & 98.68 & 98.14 & 99.75 & 100.71 & 99.86 & & & & & \\
\hline
\end{tabular}

10) of this garnet, 2.5 weight percent, is distinctive and is diagnostic of the variant of andradite known as melanite. Abundances of ferrous and ferric iron in this garnet (table 10) were computed by stoichiometry.

\section{Discussion}

\section{Tectonic Setting}

Granitoid magmas generated in various tectonic settings have distinct geochemical signatures (Pearce and others, 1984). Trace-element abundances in igneous rocks of the central Big Belt Mountains, in particular those of $\mathrm{Rb}, \mathrm{Y}$, and $\mathrm{Nb}$, are similar to those of subduction-related arc rocks (fig. 13). Gill (1981) suggested that negative $\mathrm{Nb}-\mathrm{Ta}$ anomalies (fig. 8A) and $\mathrm{Ba} / \mathrm{Ta}$ ratios greater than 450 (tables 3 and 4), both of which are characteristic of igneous rocks in the central Big Belt Mountains, are diagnostic of magmas generated in a subduction-related arc setting. Major oxide compositions of the Big Belt Mountains igneous rocks form a curvilinear array that parallels, but is displaced slightly below, that characteristic of Cascade arc calc-alkaline rocks (fig. 14). Chondrite-normalized extended trace-element patterns for igneous rocks of the central Big Belt Mountains are similar to those of mid-Tertiary volcanic rocks of the southern Great Basin (for example, du Bray, 1995b), whose origin is similarly considered to be related to diffuse back-arc volcanism that followed a period of shallow subduction (Coney and Reynolds, 1977). These compositional characteristics are all consistent with a mantle-dominated genesis of central Big Belt Mountains intrusions in a back-arc, subduction-related setting.
A back-arc, subduction-related origin for igneous rocks of the central Big Belt Mountains is consistent with the presence of voluminous, Late Cretaceous through early Tertiary igneous rocks throughout Idaho (Hyndman and Foster, 1989) and western Montana. Late Cretaceous magmatism in western Montana and eastern Idaho, including that in the Idaho and Boulder batholiths, can be placed in the context of a magmatic belt that extended southward and eastward during midCretaceous time (Coney and Reynolds, 1977; Lipman, 1992). Igneous rocks in the central Big Belt Mountains are among the easternmost manifestations of Late Cretaceous magmatism in this region. The Sliderock Mountain stratovolcano, another inferred back-arc magmatic center (du Bray and Harlan, 1998), is about $160 \mathrm{~km}$ southeast of and about 10 million years older than intrusions of the central Big Belt Mountains. Relative age and geographic relations between these two centers are consistent with renewed, westwardsweeping back-arc magmatism (following rapid, shallow subduction during the mid-Cretaceous) related to slower, increasingly steep subduction and westward retreat of the subducted slab hingeline through time.

\section{Origin of Compositional Variation in Granodiorite and Quartz Monzodiorite}

Magmatic compositional variation can result from a number of petrogenetic mechanisms, including magma mixing, wallrock stoping and assimilation, varying degrees of partial melting, and fractional crystallization. Physical and geochemical evidence was used to evaluate the contribution of these processes to evolution of compositional variation within igneous 


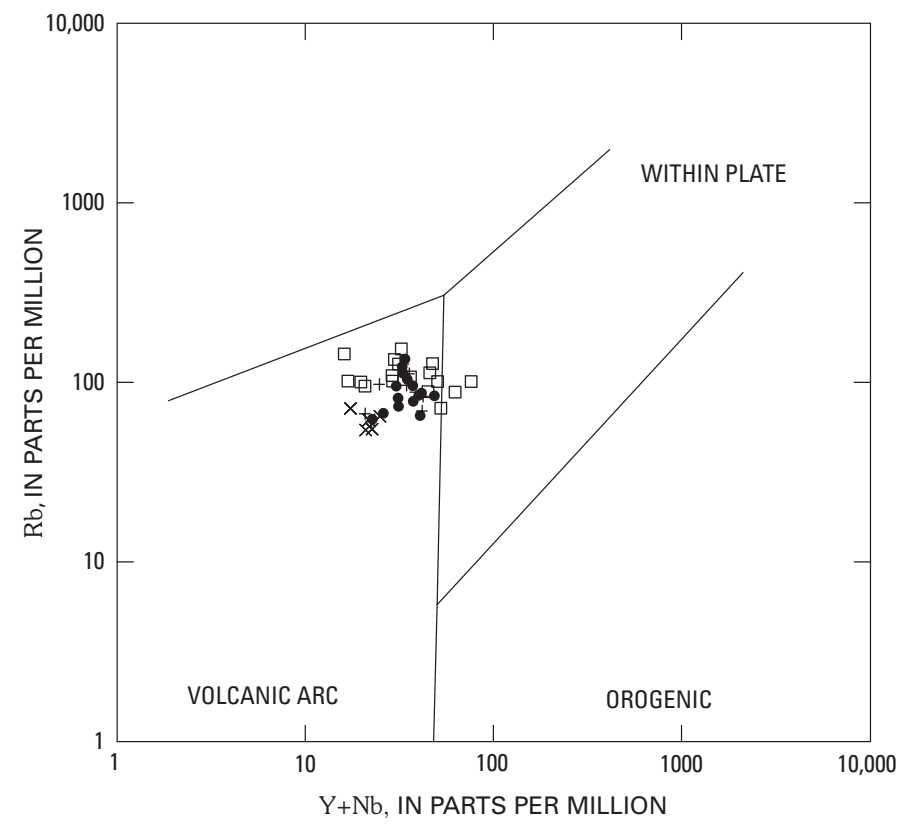

EXPLANATION

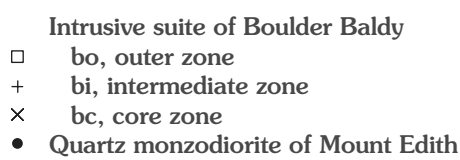

Figure 13. Trace-element tectonic-setting-discrimination variation diagram for intrusions of central Big Belt Mountains. Tectonic setting-composition boundaries from Pearce and others (1984).

rocks of the central Big Belt Mountains. Magma mixing was ruled out, in part because the intrusions lack mafic enclaves that are common in granitoids intruded by compositionally distinct magma (Vernon, 1984; Frost and Mahood, 1987). The igneous rocks are also free of wallrock xenoliths that would result from the stoping-assimilation process. Chemical data also preclude mixing as a source of the stock's compositional variation. In contrast to the intrusion compositions that result from two-component mixing, abundance variation patterns for the Big Belt Mountains intrusions are not linear.

The possibility that compositional variation within the intrusions resulted from varying degrees of partial melting was eliminated primarily on the basis of chemical considerations. Allegre and Minster (1978) demonstrated that abundance ratios of highly and moderately incompatible trace elements can be used to help identify processes responsible for compositional variation. This observation can be applied to central Big Belt Mountains intrusions by consideration of Th and Sm abundance variations. Their $\mathrm{Th} / \mathrm{Sm}$ ratios are essentially constant regardless of Th abundances (fig. 15 and table 4). Open-system behavior, such as varying degrees of partial melting or magma mixing involving sequential inputs, is inconsistent with the essentially constant $\mathrm{Th} / \mathrm{Sm}$ ratios characteristic of the Big Belt Mountains intrusions. These compositional variations are, however, consistent with development of compositional variation by closed system fractional crystallization, which causes highly

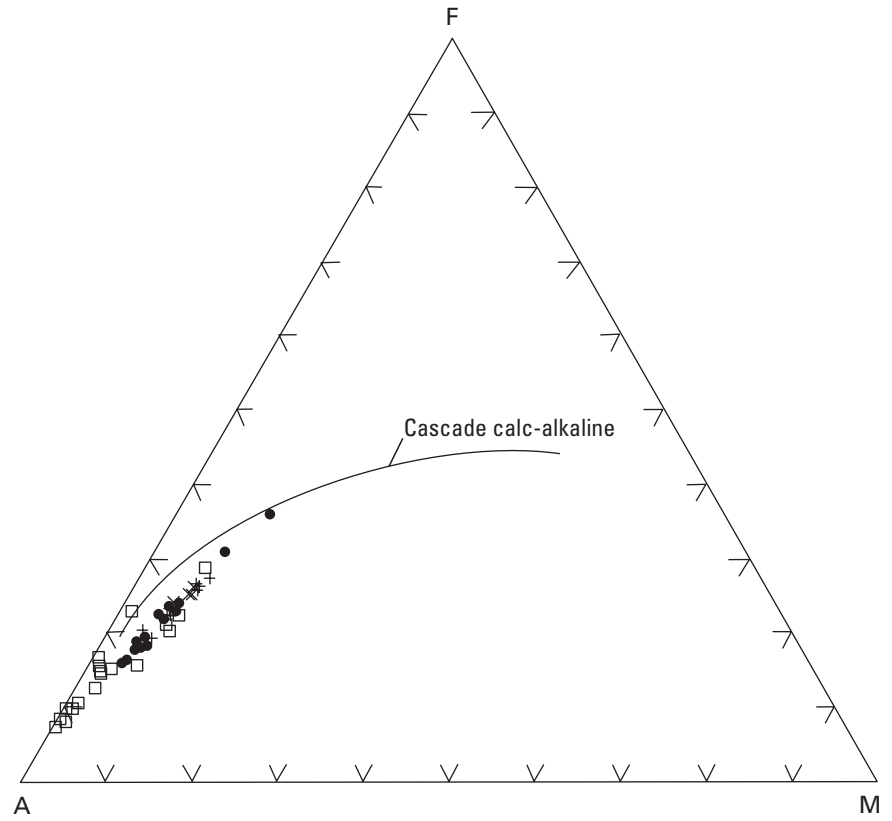

EXPLANATION

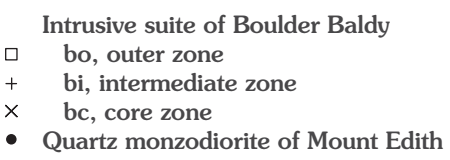

Figure 14. Ternary AFM $\left(\mathrm{Na}_{2} \mathrm{O}+\mathrm{K}_{2} \mathrm{O}-\mathrm{Fe} \mathrm{O}^{*}-\mathrm{MgO}\right)$ diagram showing compositions of central Big Belt Mountains intrusions. Cascade calcalkaline trend line from Irvine and Baragar (1971).

versus moderately incompatible trace-element abundance ratios to be essentially constant as highly incompatible trace-element abundances increase.

In order to evaluate crystal fractionation as a process responsible for development of magmatic compositional variation in biotite granodiorite and quartz monzodiorite intrusions of the central Big Belt Mountains, whole rock and mineral compositions of eight representative samples were used as reactants and products in mixing model calculations. Compositions of the quartz monzonite of the intrusive suite of Boulder Baldy were not modeled because these rocks do not preserve verifiably magmatic characteristics. Because of the compositional overlap and similarity between quartz monzodiorites of Mount Edith and the intrusive suite of Boulder Baldy, their compositions were modeled as part of a single, potential fractionation series. The composition of the most mafic, noncumulate sample of the subset (202934) was presumed to be parental to each successively less mafic (daughter) sample (202937, 202954, 202854, 202947, 202871, and finally 202946). A least-squares mixing calculation (PETMIX) was used to determine the weight fractions of minerals (with compositions like those contained in the parent sample) to be subtracted to produce a derivative liquid with a composition like that of each daughter sample (table 11). The purpose of these calculations is to demonstrate that all parts of the stock are plausibly related by fractionation of reasonable amounts of their constituent minerals. 


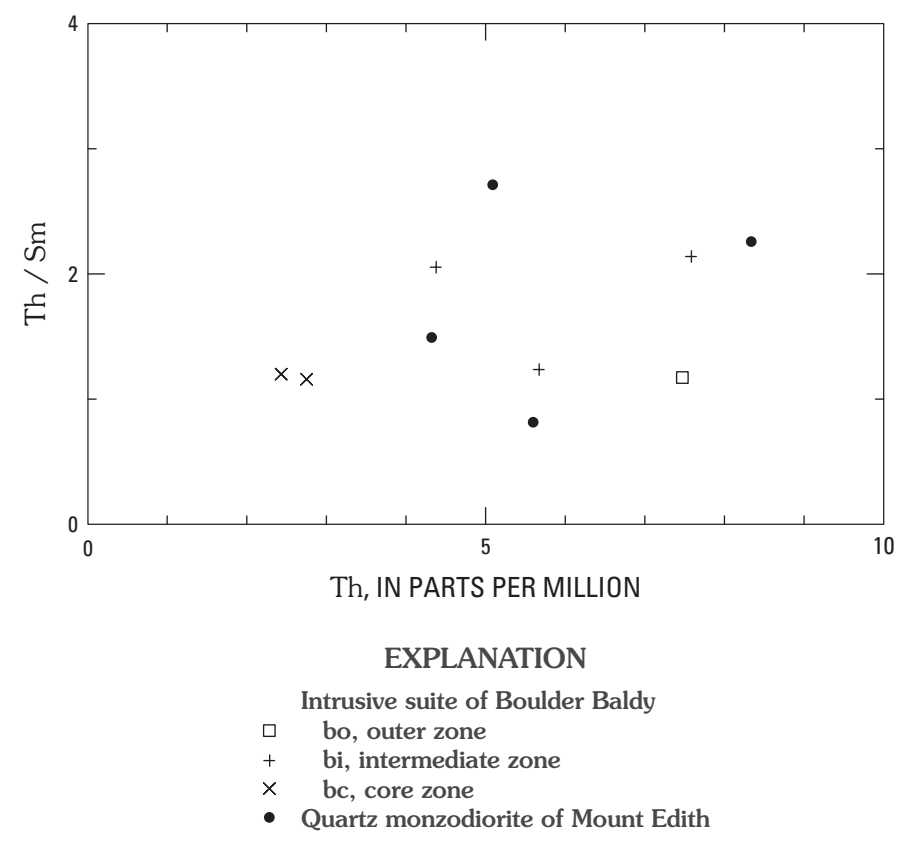

Figure 15. Th/Sm vs. Th variation diagram showing compositions of central Big Belt Mountains intrusions.

Major element least-squares crystal fractionation-mixing models are viable when the quantity $\Sigma r^{2}$ (table 11 ), the sum of the squares of each major oxide residual, is less than a value ascribed to analytical uncertainty (approximately 0.5 weight percent). Low to very low $\Sigma r^{2}$ values for six Big Belt mixing models indicate that all parts of the associated intrusions could have been generated by fractionation of constituent minerals. In both cases for which the quantity $\Sigma \mathrm{r}^{2}$ is greater than 0.2 (table 11), the majority of each $\Sigma r^{2}$ value results from large $\mathrm{Na}_{2} \mathrm{O}$ residuals. Because $\mathrm{Na}_{2} \mathrm{O}$ is notoriously mobile, these slightly elevated $\Sigma \mathrm{r}^{2}$ values probably reflect late hydrothermal alteration or weathering processes rather than true misfits between observed and modeled compositions. The composition of the most evolved sample (202946) is the silicate liquid whose modeled composition results from crystallization and removal of about 68 percent solids from the presumed parental liquid, whose composition is represented by sample 202934 (table 11). The composition of cumulate sample 202940 could have resulted from addition (as cumulates) of minerals with compositions like those found in sample 202940 to a silicate liquid with a composition like that of the presumed parent composition (202934).

Trace-element abundances for each representative sample were normalized to that of the most primitive, presumed parental sample (202934) and plotted on extended trace-element diagrams to evaluate trace-element behavior during the fractionation process just described. These patterns (fig. 16) are essentially parallel, cluster relatively tightly, and include minor superimposed positive $\mathrm{Th}$ and $\mathrm{Zr}$ anomalies. Th and $\mathrm{Zr}$ appear to have been almost equally partitioned between crystals and residual silicate liquid; their abundances in successively more evolved rocks remained essentially constant, whereas those of other trace elements decreased with differentiation. These patterns show that overall trace-element abundances decrease systematically with increasing $\mathrm{SiO}_{2}$ contents (differentiation). That none of the evaluated trace elements (including $\mathrm{Rb}$, which is usually highly incompatible (Hanson, 1978)), display well-developed incompatibility is somewhat remarkable. All these trace elements were preferentially partitioned from the residual silicate liquid into solid phases, particularly into feldspar and biotite, during solidification.

Abundances of $\mathrm{Sr}, \mathrm{K}$, and $\mathrm{Rb}$ are principally controlled by feldspar (Hanson, 1978); abundances of strontium, which typically acts as a strongly compatible element, and rubidium are respectively most and least depleted by feldspar fractionation. As feldspar fractionation and differentiation proceed, $\mathrm{K}$ and to

Table 11. Results of major oxide least-squares crystal fractionation-mixing modeling for intrusive rocks from central Big Belt Mountains.

[Numbers under mineral headings (PL, plagioclase; KF, potassium feldspar; $\mathrm{HB}$, magnesio-hornblende; CP, clinopyroxene; MG, magnetite; TI, titanite; AP, apatite) are mass percentages of each phase removed in each fractionation step]

\begin{tabular}{|c|c|c|c|c|c|c|c|c|c|}
\hline \multirow[b]{2}{*}{$\begin{array}{l}\text { Derived } \\
\text { composition }\end{array}$} & \multicolumn{8}{|c|}{$\begin{array}{l}\text { Weight fractions, in percent, of minerals } \\
\text { fractionated from parent liquid }\end{array}$} & \multirow[b]{2}{*}{$\begin{array}{c}\Sigma r^{2} \\
\text { Residual }\end{array}$} \\
\hline & $\mathrm{PL}$ & $\mathrm{KF}$ & HB & $\mathrm{CP}$ & $M G$ & $\mathrm{TI}$ & AP & $\mathrm{F}$ & \\
\hline 202946 & & & & & & & & 32.49 & 0.042 \\
\hline 202871 & 39.02 & 18.89 & 7.34 & -- & 1.30 & 0.73 & 0.23 & 33.88 & 0.239 \\
\hline 202947 & 39.91 & 15.99 & 6.14 & -- & 1.38 & 0.77 & 0.34 & 34.64 & 0.040 \\
\hline 202854 & 37.93 & 17.94 & 7.25 & -- & 1.22 & 0.72 & 0.29 & 35.69 & 0.079 \\
\hline 202954 & 36.68 & 18.53 & 6.90 & -- & 1.29 & 0.71 & 0.20 & 44.87 & 0.091 \\
\hline 202937 & 30.48 & 17.25 & 5.59 & -- & 1.00 & 0.58 & 0.23 & 59.74 & 0.305 \\
\hline 202934 & 23.82 & 9.45 & 3.64 & 1.97 & 0.75 & 0.48 & 0.16 & & \\
\hline \multicolumn{10}{|c|}{ CUMULATE: } \\
\hline 202940 & 11.26 & -- & 1.34 & 4.80 & 1.77 & 0.82 & 1.34 & 79.39 & 0.159 \\
\hline
\end{tabular}




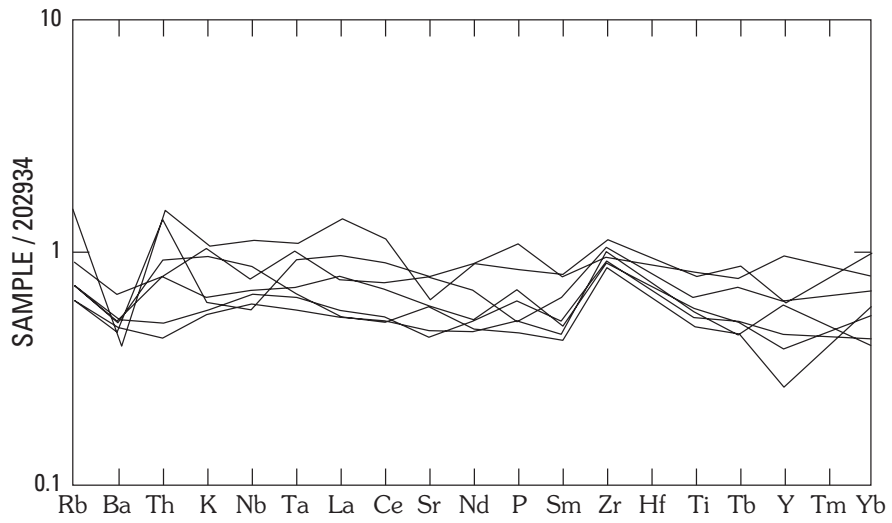

Figure 16. Extended trace-element abundance diagram showing compositions of intrusions in central Big Belt Mountains; abundances normalized to that of most primitive sample (202934).

an even greater extent, $\mathrm{Rb}$ are concentrated in the residual liquid relative to Sr. Compositions plotting nearest the $\mathrm{Sr}$ apex of the $\mathrm{Rb}-\mathrm{K} / 100-\mathrm{Sr}$ ternary diagram (fig. 17) represent the least evolved magmas. In samples of granodiorite and quartz monzodiorite from the central Big Belt Mountains, compositions plot along a short array whose origin lies near the Sr apex; they define a narrow compositional range. Mean abundance of $\mathrm{Sr}$ in the eight representative samples is $860 \pm 160 \mathrm{ppm}$. Because plagioclase is the principal residence of $\mathrm{Sr}$, tight clustering of $\mathrm{Sr}$ abundances indicates that plagioclase fractionation did not strongly influence $\mathrm{Sr}$ abundances in compositional evolution of the central Big Belt Mountains intrusions. The position, relative to more evolved rocks (du Bray and others, 1988; du Bray, 1995b, for example), and the shortness of the array also confirm that compositional evolution of central Big Belt Mountains igneous rocks was not strongly influenced by plagioclase fractionation. The shortness of the compositional evolution array on the $\mathrm{Rb}-\mathrm{K} / 100-\mathrm{Sr}$ ternary diagram (fig. 17) probably results from a relatively low distribution coefficient for $\mathrm{Sr}$ in plagioclase $\left(\mathrm{D}_{\mathrm{Sr}}\right)$ but high $\mathrm{Sr}$ abundances, relative to those of $\mathrm{Rb}$ and $\mathrm{K}$ (table 3 ). Fractionation of plagioclase with a somewhat low $\mathrm{D}_{\mathrm{Sr}}$ (for instance, less than 1.45 (Hanson, 1978)) from a very Sr-rich magma causes the abundance of $\mathrm{Sr}$, relative to those of $\mathrm{K}$ and $\mathrm{Rb}$, in residual magma to be little affected.

Abundances of REE in granodiorite and quartz monzodiorite from the central Big Belt Mountains, normalized to that of the most primitive (presumed parental) sample (202934), yield values near or slightly less than 1.0. Resulting patterns (fig. 18) are essentially parallel and flat; therefore, the REE were minimally fractionated during differentiation. Consequently, REE bulk distribution coefficients for the fractionation assemblage must have been slightly more than about one. The absence of REE fractionation indicates that variably REE enriched accessory minerals did not play a major role in chemical evolution of these igneous rocks. The lack of progressively larger negative Eu anomalies with increasing differentiation is enigmatic given that plagioclase was a fractionating phase (table 11). Intrusions of the central Big Belt Mountains may have solidified under relatively oxidizing conditions, thereby favoring trivalent europium over the divalent form that readily substitutes for calcium in

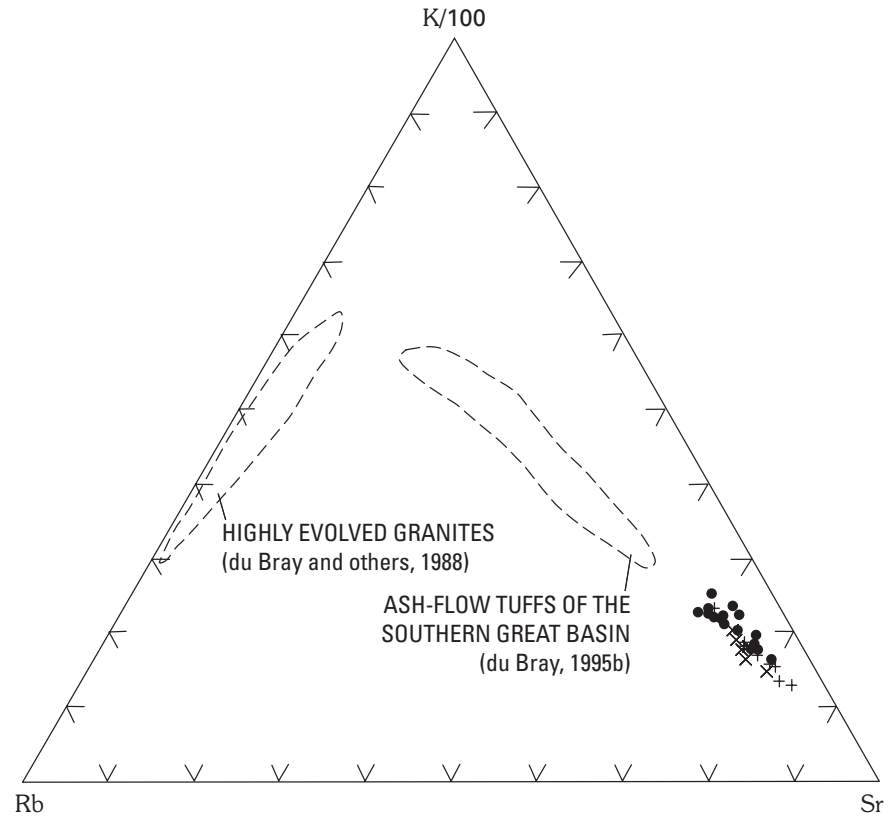

EXPLANATION

Intrusive suite of Boulder Baldy

bi, intermediate zone

$\times \quad$ bc, core zone

- Quartz monzodiorite of Mount Edith

Figure 17. Ternary variation diagram showing the relative proportions of $\mathrm{Rb}, \mathrm{K}$, and $\mathrm{Sr}$ in granodiorite and quartz monzodiorite in central Big Belt Mountains.

plagioclase. The presence of magnetite (as the sole iron oxide mineral in these intrusions) and abundant titanite (as opposed to ilmenite), both of which contain more ferric than ferrous iron, further suggests the prevalence of oxidizing conditions. Mineral-melt distribution coefficient patterns for hornblende and apatite are characterized by negative Eu anomalies (Hanson, 1980); their fractionation (table 11) causes residual liquid Eu enrichment, which may also have counteracted any tendency toward residual-liquid Eu depletion caused by plagioclase fractionation.

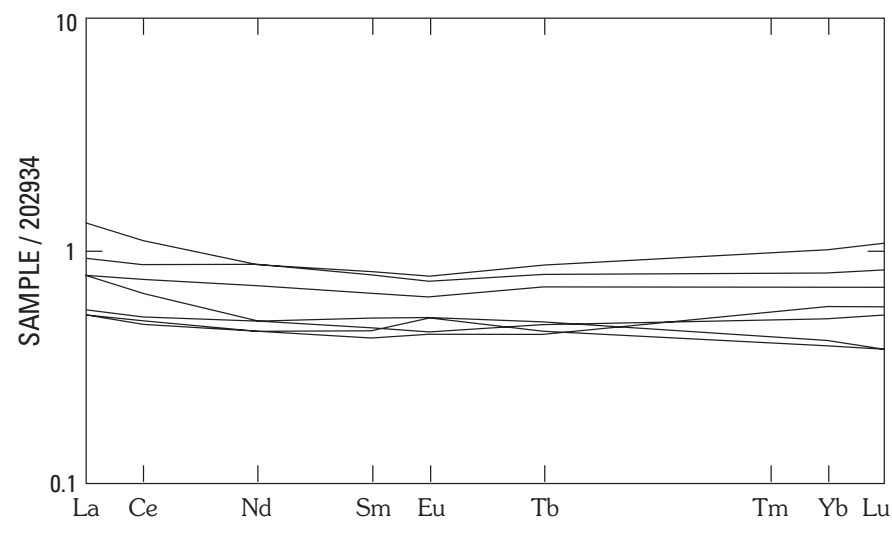

Figure 18. Rare-earth element diagram showing compositions of central Big Belt Mountains intrusions; abundances normalized to that of most primitive sample (202934). 
If crystal fractionation is accepted as a viable process for development of compositional variation in intrusions of the central Big Belt Mountains, magma reservoir processes that result in crystal fractionation must then be identified. One of the most widely accepted models for genesis of concentrically zoned plutons of the Sierra Nevada batholith involves fractional sidewall crystallization (Sawka and others, 1990). This solidification model is invoked for magma chambers whose cooling is inferred to proceed from the margin inward by conductive heat loss to host rock forming the chamber walls. As cooling proceeds, mafic silicate minerals, calcic plagioclase, and accessory minerals crystallize along the chamber walls.

A variant of the fractional sidewall crystallization model may be responsible for development of progressively more evolved magmas represented by igneous rocks of the central Big Belt Mountains. Sawka and others (1990) described processes of interstitial liquid migration toward the top of the chamber that, lacking evidence of vertical zonation, seem inapplicable to evolution of igneous rocks in the central Big Belt Mountains. Instead, melt in the associated reservoir may have become more evolved by removal of components necessary for sidewall crystallization of refractory phases. Continuous sidewall crystallization and mixing between refractory-component-depleted sidewall melt and undepleted melt inboard from the crystallization front could have produced an inward-crystallizing magma reservoir that became more evolved from the walls inward. The mafic border phase of the quartz monzodiorite of Mount Edith may represent weakly developed cumulates that formed by a combination of sidewall crystallization and filter pressing along the margins of the magma reservoir.

\section{Genesis of Quartz Monzonite in the Outer Zone}

Textural features, erratic compositional variation, unusual compositions, and mineralogy of the quartz monzonite that forms the outer zone of the intrusive suite of Boulder Baldy suggest that these outer zone rocks do not retain primary magmatic characteristics. A single sample (202945) from near the western contact of the outer zone with enclosing Newland Formation rocks (fig. 1) consists of quartz monzodiorite identical to that exposed about $1.5 \mathrm{~km}$ to the east in the intermediate zone of the intrusive suite. Rock represented by this sample is interpreted as belonging to an isolated domain, with a primary magmatic origin, that was minimally affected by alteration that variably altered rock forming the remainder of the outer zone. All rock in the outer zone is interpreted as the alteration and (or) recrystallization product of a quartz monzodiorite protolith. The extent of alteration and recrystallization is highly variable in the outer zone and is correlated with compositional variation. Specifically, the most and least altered and recrystallized samples are characterized by compositions that plot respectively farthest and nearest from trends (fig. 5) defined by granodiorite and quartz monzodiorite of the central Big Belt Mountains.

The abundance of fluorite throughout the quartz monzonite zone, both as disseminated grains and as veinlets, indicates that rock in this zone has extensively interacted with a fluorine-rich fluid. Further evidence of the importance of fluorine-bearing solutions in the genesis of outer zone rocks is presented by Sahinen (1962), who described fluorite veins in the intrusive suite. The abundance of aplite-pegmatite intergrowths and the presence of zones of densely packed, euhedral perthite phenocrysts also indicate that these rocks have interacted extensively with hydrothermal fluids. The fluids responsible for alteration of the quartz monzodiorite caused magmatic hornblende and plagioclase to be replaced by sodic pyroxene and alkali feldspar, respectively. These reactions are in accord with the absence of hornblende, diminished plagioclase abundances, and somewhat elevated alkali feldspar abundances in pyroxene-bearing samples of the quartz monzonite. Rocks affected by this initial, weak metasomatic alteration retain primary magmatic features, including interlocking grain fabrics and seriate textures.

Abundant rock in the outer zone lacks petrographic features indicative of a primary magmatic origin. These rocks lack plagioclase, are primarily composed of densely packed coarsegrained to very coarse grained perthite crystals, and contain andradite garnet. Many of these rocks contain no mafic silicate minerals. However, one group of samples is probably transitional between quartz monzonite that contains sodic pyroxene and that which contains andradite; these samples contain unstable and reacted sodic pyroxene. These petrographic features reflect intense hydrothermal alteration that resulted in replacement of sodic pyroxene by andradite. Sodic pyroxene, remaining plagioclase, and magnetite reacted with a hydrothermal fluid to form andradite, alkali feldspar, and excess $\mathrm{MgO}$ and $\mathrm{SiO}_{2}$. These reactions are in accord with the absence of aegirineaugite, plagioclase, and magnetite, and significantly elevated alkali feldspar abundances in andradite-bearing samples of the quartz monzonite.

These reactions yield excess $\mathrm{MgO}$; samples from the outer zone are distinctly $\mathrm{MgO}$ depleted (fig. 5) relative to unaltered granodiorite and quartz monzodiorite of the central Big Belt Mountains. The reaction by which andradite was produced also resulted in excess $\mathrm{SiO}_{2}$. Fluids responsible for metasomatic alteration of rock now exposed in the outer zone probably transported $\mathrm{MgO}$ and $\mathrm{SiO}_{2}$ out of the magmatic system into surrounding wallrocks. The presence of numerous large quartz veins in Newland Formation rocks peripheral to the intrusive suite of Boulder Baldy indicates that $\mathrm{SiO}_{2}$ was expelled from the magmatic system, probably in response to the replacement of sodic pyroxene by andradite and other desilication reactions. Exploration studies at Miller Mountain (fig. 1) indicate that the stock exposed there contains a small (at least 11 million tons of ore containing 310,000 oz gold), subeconomic gold deposit (Greg Whitman, Pegasus Gold Corporation, oral commun., 1993). Genesis of the gold-bearing quartz stockwork at Miller Mountain probably reflects these same processes. The presence of numerous smaller gold prospects along contacts between central Big Belt Mountains intrusions and enclosing Newland Formation rocks (du Bray, 1995a) suggests that the hydrothermal fluid responsible for alteration of quartz monzodiorite to quartz monzonite precipitated gold and other metals near bounding contacts, where physico-chemical gradients were steepest.

The source of the fluids responsible for the alteration described herein is uncertain. Because rocks of the Newland Formation are fine grained and have low porosity, they are an 
unlikely fluid source. However, the Newland Formation rocks contain numerous fractures that could have served as fluid conduits. The abundance of fluorine in the hypothesized fluid phase, as indicated by accessory fluorite, and the presence of aplite-pegmatite intergrowths associated with altered rock indicate a probable magmatic origin. Solidification of the central Big Belt Mountains intrusions almost certainly involved latestage fluid-phase exsolution. The presence of a variably developed, fine-grained, anhedral groundmass among many samples of the quartz monzonite zone suggests that final solidification of magma represented by these rocks involved rapid nucleation, possibly in response to pressure quenching events. Pressure quenching causes rapid exsolution, which in turn may have initiated variably intense alteration and recrystallization of quartz monzodiorite to rock represented by the outer zone of the intrusive suite of Boulder Baldy.

Many features of the altered rocks associated with the intrusions of the central Big Belt Mountains are similar to those of granitoid rocks that have been affected by the alteration that accompanies late-stage magmatic and hydrothermal processes that are characteristic of Climax-type molybdenum deposits (White and others, 1981). This observation is consistent with the association, identified by Lang and others (2000, fig. 7), between moderately reduced intrusion-related gold systems and molybdenum and tungsten deposits associated with evolved and moderately reduced intrusions emplaced in subduction-related continental settings. The geochemical, petrographic, and tectonic characteristics of the central Big Belt Mountains intrusions and associated mineralized rocks clearly are similar to those identified in intrusions and mineralized rock associated with Climax-type molybdenum deposits as well as those of intrusionrelated gold deposits. Consequently, the central Big Belt Mountains intrusions and associated mineralized rocks may represent a genetic transition between the two mineral deposit types.

Development of the altered, outer zone of the intrusive suite of Boulder Baldy appears to have resulted from magmatic exsolution processes common to Climax-type molybdenum deposits. The relationship between these hydrothermal processes and genesis of small gold deposits, such as that at Miller Mountain, is uncertain but represents a research avenue that is critical to determining the genesis of granite-related gold deposits.

Considering the proposed consanguinity of all central Big Belt Mountains intrusions, the fact that the quartz monzodiorite of Mount Edith lacks post-magmatic hydrothermal alteration features is somewhat puzzling. However, most of the quartz monzodiorite of Mount Edith lacks a fine-grained, anhedral groundmass, which suggests that this intrusion did not experience the extent of pressure quenching and attendant rapid fluid exsolution that apparently played a major role in solidification of the quartz monzodiorite of the intrusive suite of Boulder Baldy. In addition, magma represented by the quartz monzodiorite of Mount Edith may have contained lower dissolved water contents than similar magma represented by the intrusive suite of Boulder Baldy and was therefore less susceptible to significant fluid exsolution during cooling. Consequently, the quartz monzodiorite of Mount Edith was not significantly altered and metasomatically recrystallized by the processes that affected quartz monzodiorite in the intrusive suite of Boulder Baldy.
The apparently large volume (assuming that surficial exposures extend to some considerable depth) of hydrothermally altered rock depicted by the outer zone of the intrusive suite is difficult to reconcile with the limited volume of fluid that typically results from solidification and attendant magmatic exsolution of calc-alkaline intrusions. Perhaps the volume of altered rock is less than would result by inferring that the altered, outer zone extends to considerable depth. In fact, although exposures are limited and field relations poorly exposed, altered, outer zone rock may only form a thin tabular carapace that overlies and marginally encloses otherwise normal intermediate zone rock. However, if altered, outer zone rock does extend to considerable depth and an additional source of abundant fluids is required, major, fluid-saturated faults may have been intersected during magma intrusion. Interaction between early-emplaced, outer zone magma and abundant structurally channelized fluid could have increased the extent of hydrothermal alteration of outer zone rocks. Isotopic analyses of samples from the outer zone could help determine whether fluids responsible for alteration and metasomatic recrystallization of rock represented by the outer zone were primarily magmatic, meteoric, or a combination of both.

\section{Conclusions}

The central Big Belt Mountains of western Montana contain several Late Cretaceous intrusions, including a pluton represented by the quartz monzodiorite of Mount Edith and a 10$\mathrm{km}$ diameter intrusive suite centered between Boulder Baldy and Boulder Mountain. The intrusive suite consists of three compositional and textural zones, of which only the inner two are considered to reflect purely magmatic processes. In contrast, with the exception of a weakly developed mafic border phase, the quartz monzodiorite of Mount Edith is generally homogeneous. ${ }^{40} \mathrm{Ar} /{ }^{39} \mathrm{Ar}$ geochronologic data indicate that these rocks crystallized before $68 \mathrm{Ma}$ (but probably between 70 and $69 \mathrm{Ma}$ ), that all are part of a single magmatic event, that regional cooling related to the magmatism in this area was complete by about $60 \mathrm{Ma}$, and that metal deposits and plutonic rocks of the central Big Belt Mountains are temporally related. Geochemical characteristics and geologic relations indicate that genesis of these calc-alkaline intrusions was related to subduction and renewed back-arc magmatism along the west edge of the late Mesozoic North America plate following a period of shallow subduction. Fractionation-mixing calculations indicate that compositional variation within granodiorite and quartz monzodiorite of the central Big Belt Mountains developed in response to varying amounts of plagioclase, clinopyroxene, hornblende, titanite, apatite, and magnetite fractionation. Crystals nucleated along the walls and roof of the cooling magma chamber while residual silicate liquid continued mixing.

In contrast, unusual textures and erratic compositional variation, characteristic of quartz monzonite in the outer zone of the intrusive suite of Boulder Baldy, probably reflect extensive metasomatic alteration and recrystallization of quartz monzodiorite by fluorine-rich, gold-bearing fluids. Intrusive rocks and 
their associated metal deposits bear striking resemblance to recently described (Lang and others, 2000) intrusion-related gold systems. Additional study, especially isotopic and fluid inclusion analyses, of the Miller Mountain deposit and the large, associated intrusion centered on Boulder Baldy could add considerably to the understanding of the genesis of this deposit type.

\section{References Cited}

Alexander, E.C., Michelson, G.M., and Lanphere, M.A., 1978, MMhb-1; A new ${ }^{40} \mathrm{Ar} /{ }^{39} \mathrm{Ar}$ dating standard: U.S. Geological Survey Open-File Report 78-701, p. 6-8.

Allegre, C.J., and Minster, J.F., 1978, Quantitative models of trace element behavior in magmatic processes: Earth and Planetary Science Letters, v. 38, p. 1-25.

Baedecker, P.A., and McKown, D.M., 1987, Instrumental neutron activation analysis of geochemical samples, in Baedecker, P.A., ed., Methods for geochemical analysis: U.S. Geological Survey Bulletin 1770, p. H1-H14.

Bateman, P.C., 1992, Plutonism in the central part of the Sierra Nevada Batholith, California: U.S. Geological Survey Professional Paper $1483,186 \mathrm{p}$.

Coney, P.J., and Reynolds, S.J., 1977, Cordilleran Benioff zones: Nature, v. 270, p. 403-406.

Dalrymple, G.B., 1979, Critical tables for conversion of K-Ar ages from old to new constants: Geology, v. 7, p. 558-560.

Dalrymple, G.B., Alexander, E.C., Lanphere, M.A., and Kraker, G.P., 1981, Irradiation of samples for ${ }^{40} \mathrm{Ar} /{ }^{39} \mathrm{Ar}$ age dating using the U.S. Geological Survey Triga reactor: U.S. Geological Survey Professional Paper 1176, $56 \mathrm{p}$.

Dalrymple, G.B., and Lanphere, M.A., 1969, Potassium-argon dating: San Francisco, Freeman, $251 \mathrm{p}$.

Daniel, Faith, and Berg, R.B., 1981, Radiometric dates of rocks in Montana: Montana Bureau of Mines and Geology Bulletin 114, $136 \mathrm{p}$.

Dodge, F.C.W., Smith, V.C., and Mays, R.E., 1969, Biotites from the central Sierra Nevada batholith, California: Journal of Petrology, v. 10, p. 250-271.

du Bray, E.A., 1995a, Geologic map showing distribution of Cretaceous intrusive rocks in the central Big Belt Mountains, Broadwater and Meagher Counties, Montana: U.S. Geological Survey Miscellaneous Field Studies Map MF-2291, scale 1:50,000.

du Bray, E.A., 1995b, Geochemistry and petrology of Oligocene and Miocene ash-flow tuffs of the southeastern Great Basin: U.S. Geological Survey Professional Paper 1559, 38 p.

du Bray, E.A., Elliott, J.E., and Stuckless, J.S., 1988, Postorogenic peraluminous granites and associated $\mathrm{Sn}-\mathrm{W}$ deposits, Kingdom of Saudi Arabia, in Taylor, R.P., and Strong, D.F., eds., Recent advances in the geology of granite-related mineral deposits: Canadian Institute of Mining and Metallurgy Special Volume 39, p. 142-156.

du Bray, E.A., and Harlan, S.S., 1996, The Big Timber stock, south-central Montana-Development of extensive compositional variation in an Eocene, arc-related intrusion by sidewall crystallization and cumulate glomerocryst remixing: Geological Society of America Bulletin, v. 108, p. 1404-1424.

du Bray, E.A., and Harlan, S.S., 1998, Geology, petrology, and age of the Cretaceous Sliderock Mountain stratovolcano, Montana: U.S. Geological Survey Professional Paper 1602, $19 \mathrm{p}$.
Elsass, Francoise, and du Bray, E.A., 1982, Energy-dispersive X-ray fluorescence spectrometry with the Kevex 7000 system: Saudi Arabian Deputy Ministry Mineral Resources Open File Report USGS-OF-02$52,53 \mathrm{p}$.

Ewart, A., 1982, The mineralogy and petrology of Tertiary-Recent orogenic volcanic rocks with special reference to the andesitic-basaltic compositional range, in Thorpe, R.S., ed., Andesites: Chichester, UK, John Wiley and Sons, p. 25-87.

Fleck, R.J., Sutter, J.F., and Elliott, D.H., 1977, Interpretation of discordant ${ }^{40} \mathrm{Ar} /{ }^{99} \mathrm{Ar}$ age spectra of Mesozoic tholeiites from Antarctica: Geochimica et Cosmochimica Acta, v. 41, p. 15-32.

Frost, T.P., and Mahood, G.A., 1987, Field, chemical, and physical constraints on mafic-felsic magma interaction in Lamarck Granodiorite, Sierra Nevada, California: Geological Society of America Bulletin, v. 99, p. 272-291.

Gill, James, 1981, Orogenic andesites and plate tectonics: New York, Springer-Verlag, $390 \mathrm{p}$.

Gualtieri, J.L., 1975a, Preliminary unedited geologic map of the Confederate Gulch area, Broadwater and Meagher Counties, Montana: U.S. Geological Survey Open-File Report 75-211, scale 1:48,000.

Gualtieri, J.L., 1975b, Geochemical data for the Confederate Gulch area, Broadwater and Meagher Counties, Montana: U.S. Geological Survey Open-File Report 75-212.

Hanson, G.N., 1978, The application of trace elements to the petrogenesis of igneous rocks of granitic composition: Earth and Planetary Science Letters, v. 38, p. 26-43.

Hanson, G.N., 1980, Rare earth elements in petrogenetic studies of igneous systems: Annual Review of Earth and Planetary Sciences, v. 8, p. 371-406.

Hyndman, D.W., and Foster, D.A., 1989, Plutonism at deep crustal levels-The Idaho batholith, Montana and Idaho, in Hyndman, D.W., ed., Cordilleran volcanism, plutonism, and magma generation at various crustal levels, Montana and Idaho: American Geophysical Union Field Trip Guidebook T337, p. 3-15.

Irvine, T.N., and Baragar, W.R.A., 1971, A guide to the chemical classification of the common volcanic rocks: Canadian Journal of Earth Sciences, v. 8, p. 523-548.

Lang, J.R., Baker, T., Hart, C.J.R., and Mortensen, J.K., 2000, Intrusionrelated gold systems: Society of Economic Geology Newsletter, v. 40, p. 1-15.

Leake, B.E., 1978, Nomenclature of amphiboles: Mineralogical Magazine, v. 42, p. 533-563.

Le Bas, M.J., Le Maitre, R.W., Streckeisen, A., and Zanettin, B., 1986, A chemical classification of volcanic rocks using the total alkali-silica diagram: Journal of Petrology, v. 27, p. 745-750.

Lipman, P.W., 1992, Magmatism in the Cordilleran United States; progress and problems, in Burchfiel, B.C., Lipman, P.W., and Zoback, M.L., eds., The Cordilleran orogen-Conterminous U.S.: Geological Society of America, The geology of North America, v. G3, p. 481-514.

Lovera, O.M., Richter, F.M., and Harrison, T.M., $1989,{ }^{40} \mathrm{Ar} /{ }^{39} \mathrm{Ar}$ thermochronometry for slowly cooled samples having a distribution of diffusion domain sizes: Journal of Geophysical Research, v. 94, p. 17919-17935.

Lyden, C.J., 1948, The gold placers of Montana: Montana Bureau of Mines and Geology Memoir 26, $152 \mathrm{p}$.

Martin, M.W., Dilles, J.H., and Proffett, J.M., 1999, U-Pb geochronologic constraints for the Butte porphyry system: Geological Society of America Abstracts with Programs, v. 31, no. 7, p. A380. 
McClernan, H.G., 1980, Metallogenic map of the White Sulphur Springs quadrangle, central Montana: Montana Bureau of Mines and Geology Map 7, 31 p.

Nelson, W.H., 1963, Geology of the Duck Creek Pass quadrangle, Montana: U.S. Geological Survey Bulletin 1121-J, 55 p., scale $1: 62,500$.

Pearce, J.A., Harris, N.B.W., and Tindle, A.G., 1984, Trace element discrimination diagrams for the tectonic interpretation of granitic rocks: Journal of Petrology, v. 25, p. 956-983.

Pegasus Gold Inc., 1992, Annual Report, p. 27.

Roddick, J.C., 1983, High precision intercalibration of ${ }^{40} \mathrm{Ar} /{ }^{39} \mathrm{Ar}$ standards: Geochimica et Cosmochimica Acta, v. 47, p. 887-898.

Rutland, Carolyn, Smedes, H.W., Tilling, R.I., and Greenwood, W.R., 1989, Volcanism at shallow crustal levels-The Elkhorn Mountains Volcanics and the Boulder batholith, southwestern Montana, in Hyndman, D.W., ed., Cordilleran volcanism, plutonism, and magma generation at various crustal levels, Montana and Idaho: American Geophysical Union Field Trip Guidebook T337, p. 16-31.

Sahinen, U.M., 1962, Fluorspar deposits in Montana: Montana School of Mines Bulletin 28, $38 \mathrm{p}$.

Samson, S.D., and Alexander, E.C., 1987, Calibration of interlaboratory ${ }^{40} \mathrm{Ar} /{ }^{39} \mathrm{Ar}$ dating standard MMhb-1: Isotope Geoscience, v. 66, p. 2734.

Sawka, W.N., Chappell, B.W., and Kistler, R.W., 1990, Granitoid compositional zoning by side-wall boundary layer differentiation-Evidence from the Palisade Crest Intrusive Suite, central Sierra Nevada, California: Journal of Petrology, v. 31, p. 519-553.

Snee, L.W., Miggins, D.P., Geissman, J.W., Reed, M.H., Dilles, J.H., and Zhang, L., 1999, Thermal history of the Butte porphyry system,
Montana: Geological Society of America Abstracts with Programs, v. 31, no. 7, p. A380.

Snee, L.W., Sutter, J.F., and Kelley, W.C., 1988, Thermochronology of economic mineral deposits; Dating the stages of mineralization at Panasqueira, Portugal by high-precision ${ }^{40} \mathrm{Ar} /{ }^{39} \mathrm{Ar}$ age spectrum techniques on muscovite: Economic Geology, v. 83, p. 335-354.

Steiger, R.H., and Jäger, E., 1977, Subcommission on geochronology; Convention on the use of decay constants in geo- and cosmochronology: Earth and Planetary Science Letters, v. 36, p. 359-362.

Streckeisen, A.L., 1973, Plutonic rocks, classification and nomenclature recommended by the IUGS subcommission on the systematics of igneous rocks: Geotimes, v. 18, no. 10, p. 26-30.

Taggart, J.E., Lindsay, J.R., Scott, B.A., Vivit, D.V., Bartel, A.J., and Stewart, K.C., 1987, Analysis of geologic materials by X-ray fluorescence spectrometry, Chapter E in Baedecker, P.A., ed., Methods for geochemical analysis: U.S. Geological Survey Bulletin 1770, p. E1E19.

Tilling, R.I., 1973, Boulder batholith, Montana-A product of two contemporaneous but chemically distinct magma series: Geological Society of America Bulletin, v. 84, p. 3879-3900.

Vernon, R.H., 1984, Microgranitoid enclaves in granites-Globules of hybrid magma quenched in a plutonic environment: Nature, v. 309, p. 438-439.

White, W.H., Bookstrom, A.A., Kamilli, R.J., Ganster, M.W., Smith, R.P., Ranta, D.E., and Steininger, R.C., 1981, Character and origin of Climax-type molybdenum deposits: Economic Geology, v. 75, p. 270-316.

Yager, D.B., and Quick, J.E., 1992, Superxap manual: U.S. Geological Survey Open-File Report 92-13, $45 \mathrm{p}$.

York, Derek, 1969, Least-squares fitting of a straight line with correlated errors: Earth and Planetary Science Letters, v. 39, p. 89-93.

Published in the Central Region, Denver, Colorado

Manuscript approved for publication October 12, 2001

Graphics by authors and Springfield and Springfield

Photocomposition by Gayle M. Dumonceaux

Edited by L.M. Carter 